\title{
Pairwise Balanced Designs whose Block Size Set Contains Seven and Thirteen
}

\author{
M. Greig \\ Greig Consulting, North Vancouver, B.C., Canada, V'7L 4R3, \\ greig@sfu.ca \\ M. Grüttmüller \\ Department of Mathematics, University of Rostock, 18051 Rostock, \\ Germany \\ martin.gruettmueller@uni-rostock.de \\ S. Hartmann \\ Department of Information Systems, Massey University, Private Bag \\ 11222, Palmerston North, New Zealand \\ s.hartmann@massey.ac.nz
}

\begin{abstract}
In this paper, we investigate the PBD-closure of sets $K$ with $\{7,13\} \subseteq K \subseteq\{7,13,19,25,31,37,43\}$. In particular, we show that $v \equiv 1 \bmod 6, v \geq 98689$ implies $v \in B(\{7,13\})$. As a preliminary result, many new 13-GDDs of type $13^{q}$ and resolvable BIBD with block size 6 or 12 are also constructed. Furthermore, we show some elements to be not essential in a Wilson bases for the PBD-closed set $\{v: v \equiv 1 \bmod 6, v \geq 7\}$.
\end{abstract}

Last compiled: June 28, 2005 


\section{Introduction}

Let $K$ be a set of positive integers. Then a pairwise balanced design $\operatorname{PBD}(v, K)$ of order $v$ with block sizes from $K$ is a pair $(V, \mathcal{B})$, where $V$ is a finite set (the point set) of cardinality $v$ and $\mathcal{B}$ is a family of subsets (called blocks) of $V$ which satisfy the following properties:

(i) every pair of distinct elements of $V$ occurs in exactly one block of $\mathcal{B}$;

(ii) if $B \in \mathcal{B}$, then $|B| \in K$.

In a sequence of three papers R.M. Wilson [25, 26, 28] developed a theory of PBD-closed sets and the notation of PBD-closure. A set $S$ of positive integers is said to be $P B D$-closed if the existence of a $\operatorname{PBD}(v, S)(v>0)$ implies that $v$ belongs to $S$. Let $K$ be a set of positive integers and let $B(K)=\{v>0: \exists \operatorname{PBD}(v, K)\}$. Then $B(K)$ is a PBD-closed set called the $P B D$-closure of $K$. According to Wilson's theory there exists a constant $c_{0}(K)$ such that designs $\operatorname{PBD}(v, K)$ exist for all $v \geq c_{0}(K)$ which satisfy the congruences $(v-1) \equiv 0 \bmod \alpha(K)$ and $v(v-1) \equiv 0 \bmod \beta(K)$, where $\alpha(K)=\operatorname{gcd}\{k-1: k \in K\}$ and $\beta(K)=\operatorname{gcd}\{k(k-1): k \in K\}$. Concerning the structure of PBD-closed sets Wilson also showed that if $S$ is a PBD-closed set, then $S$ is eventually periodic with period $\beta(S)$; that is, there exists a constant $c_{0}(S)$ such that for every $k \in S,\left\{v: v \geq c_{0}(S), v \equiv k \bmod \beta(S)\right\} \subseteq$ $S$.

The theory of PBD-closed sets is a powerful tool for investigations of combinatorial structures, a finite number of known examples of a certain set of objects can establish the existence of the entire set of these objects, for examples, see [22, p. 203] and [27].

Unfortunately, the constant $c_{0}(K)$ is not known in general. Although Wilson's proof is somehow constructive, the estimate of the constant is very large. Therefore, one attempts to determine $B(K)$ for given $K$ as accurately as possible, for a survey, see, for example, [22, Tables 3.17, 3.18].

In this paper, we investigate the PBD-closure of sets $K$ with $\{7,13\} \subseteq$ $K \subseteq\{7,13,19,25,31,37,43\}$. Our motivation to consider these sets $K$ comes from a problem on pan-orientable block designs. In [19] it is shown that the set of values $v$ which admit a pan-orientable block design $(v, 4,2)$ is a PBDclosed set containing 7 and 13 and is a subset of $N_{1,6}=\{v: v \geq 6, v \equiv$ $1 \bmod 6\}$. We are able to give in Section 5 an upper bound on $c_{0}(\{7,13\})$ : $c_{0}(\{7,13\}) \leq 98689$. More precise upper bounds are obtained when looking at the residues modulo 42. In Table 1 we present the largest possible exception in each residue class $6 t+1$ modulo $42,0 \leq t<7$. Note that the largest exceptions in the residue classes 1,7 or 13 modulo 42 are small 


\begin{tabular}{lrrrrrrr}
\hline $6 t+1$ modulo 42 & 1 & 7 & 13 & 19 & 25 & 31 & 37 \\
\hline Largest exception & 2605 & 1645 & 14293 & 82549 & 98683 & 91507 & 88447 \\
\hline
\end{tabular}

Table 1: Largest possible exception in each residue class $6 t+1$ modulo 42

compared to largest exceptions for $19,25,31$ or 37 modulo 42 . Therefore, it seems opportune to include in $K$ the smallest values from each fibre modulo 42 in order to eliminate numbers that are in the list of exceptions for $B(\{7,13\})$. So, we study in Section 6 the PBD-closure of sets $K$ where $\{7,13\} \subset K \subseteq\{7,13,19,25,31,37,43\}$.

The constructions we used to establish these results are partially taken from Mullin and Stinson [23] which determined the PBD-closure of $P_{1,6}$, where $P_{1,6}$ is defined to be the set of prime powers congruent 1 modulo 6 . They showed that $B\left(P_{1,6}\right) \cup Q=N_{1,6}$, where $Q$ is a set of 31 (possible) exceptions. Subsequently, their result was improved by Greig [17] who removed 9 of the values in $Q$. In Section 4 a number of new constructions, which are based on Wilsons Fundamental Construction, are introduced. As ingredients for these constructions we will construct many new 13-GDDs of type $13^{q}$ and (resolvable) BIBD with block size 6,12 or 13 .

As a consequence of Wilson's existence theory it follows that if $S$ is a PBD-closed set, then there exists a finite subset $J \subseteq S$ such that $S=B(J)$. Such a set $J$ is said to be a finite basis or Wilson basis for the closed set $S$. An element $x \in J$ is called essential if and only if $x \notin B(J \backslash\{x\})$. Mullin [21] determined a first Wilson basis for the set $N_{1,6}=B(\{7,13,19,25,31,37$, $43,55,61,67,73,79,97,103,109,115,121,127,139,145,157,163,181$, 193, 199, 205, 211, 223, 229, 235, 241, 253, 265, 271, 277, 283, 289, 295, 307, 313, 319, 331, 349, 355, 361, 367, 373, 379, 391, 397, 409, 415, 421, 439, 445, 451, 457, 487, 493, 499, 643, 649, 655, 661, 667, 685, 691, 697, 709, 727, 733, 739, 745, 751, 781, 787, 811, 1063, 1069, 1231, 1237, 1243, 1249, 1255, 1315, 1321, 1327, 1543, 1549, 1567, 1579 1585, 1783, 1789, 1795, 1801, $1819,1831\})$. Independently, Du [14] and Greig [17] removed the elements 223, 253, 295, 307, 361, 367, 379, 421, 439, 655, 727, 1231, 1237, 1243, 1249, $1255,1543,1549,1585,1783,1789,1795,1801,1819$ and 1831 from the finite basis for $N_{1,6}$. We will improve this result in Section 4 by showing that 4 further values 1063, 1069, 1567 and 1579 are not essential. 


\section{Preliminaries}

In this section, we give some definition and notations as well as some preliminary results which will be used in the sequel. We refer the reader to [9] and [12] for undefined terms as well as a general overview of design theory.

Fundamental to our constructions are a number of designs which we define now. A group-divisible design (GDD) is a triple $(V, \mathcal{G}, \mathcal{B})$ where $V$ is a set of points, $\mathcal{G}$ is a partition of $V$ into groups and $\mathcal{B}$ is a collection of subsets of $V$ (called blocks) such that any pair of distinct points in $V$ occurs together either in some group or in exactly one block, but not both. A $K$-GDD of type $g_{1}^{t_{1}} g_{2}^{t_{2}} \ldots g_{r}^{t_{r}}$ is a GDD in which each block has size from the set $K$ and in which there are $t_{i}$ groups of size $g_{i}, i=1,2, \ldots, r$. We will denote a $\{k\}$-GDD as a $k$-GDD. Group divisible designs are useful as ingredients in Wilson's Fundamental Construction.

Construction 2.1 Let $(V, \mathcal{G}, \mathcal{B})$ be a $G D D$ and let $w: V \rightarrow \mathbb{Z}^{+} \cup\{0\}$ ( $w$ is called a weight function). Suppose that for each block $B \in \mathcal{B}$ there is a $K$ $G D D$ of type $\{w(x): x \in B\}$. Then there is a $K-G D D$ of type $\left\{\sum_{x \in G_{i}} w(x)\right.$ : $\left.G_{i} \in \mathcal{G}\right\}$.

A transversal design $\operatorname{TD}(k, n)$ is a $k$-GDD of type $n^{k}$. It is well-known that the existence of a TD $(k, n)$ is equivalent to that of $k-2$ mutually orthogonal Latin squares (MOLS) of side $n$. Also, it is well known that for all prime powers $q$ there is a $\operatorname{TD}(q+1, q)$. Our source of TDs is the following result of MacNeish [20].

Lemma 2.2 If a $T D(k, m)$ and a $T D(k, n)$ exist, then a $T D(k, m n)$ exists.

Moreover, we use the full knowledge of the updated MOLS table $[4,13,6]$ which provides a list of lower bounds on the number of MOLS of all orders up to 10000. In particular, $\operatorname{TD}(14, m)$ play an important role in one of our constructions. Thus, we mention the following result [4, Table 2.68].

Lemma 2.3 If $m \geq 6713$ and $m \equiv 0 \bmod 7$, or if $m \geq 3567$ and $m$ is odd, or if $m \geq 7289$, then there is a $T D(14, m)$.

We also require the notion of incomplete designs. An incomplete pairwise balanced design $\operatorname{IPBD}(v, h, K)$ is a triple $(V, Y, \mathcal{B})$ where $V$ is a set of $v$ points, $Y$ is a subset of $V$ of size $h$ ( $Y$ is called the hole), and $\mathcal{B}$ is a collection of subsets of $V$ (blocks) such that

(i) any pair of distinct elements of $V$ occur together either in the hole $Y$ or in exactly one block of $\mathcal{B}$, but not both; 
(ii) if $B \in \mathcal{B}$, then $|B| \in K$.

Suppose $(V, Y, \mathcal{B})$ is an $\operatorname{IPBD}(v, h, K)$ and $(Y, \mathcal{Y})$ is a $\operatorname{PBD}(h, K)$. Then we say that $(V, \mathcal{B} \cup \mathcal{Y})$ is a PBD with a flat $Y$ of order $h$. Note that in any PBD any block can be considered to be a flat.

Lemma 2.4 Suppose there is an $\operatorname{IPBD}(v, w, K)$ and an $\operatorname{IPBD}(w, h, K)$. Then there is an $\operatorname{IPBD}(v, h, K)$.

Proof. Fill the hole $W$ of the $\operatorname{IPBD}(v, w, K)$ with the blocks of the $\operatorname{IPBD}(w, h, K)$ defined on the point set $W$ to obtain the desired design.

An incomplete transversal design $\operatorname{ITD}(k ; n, m)$ is a quadruple $(V, Y, \mathcal{G}, \mathcal{B})$ where $V$ is a set of $k n$ points, $Y$ is a subset of $V$ of size $k m$ ( $Y$ is called the hole), $\mathcal{G}$ is a partition of $V$ into $k$ groups, each of size $n$, and $\mathcal{B}$ is a collection of $k$-subsets of $V$ (blocks) such that

(i) for each group $G_{i} \in \mathcal{G},\left|G_{i} \cap Y\right|=m$,

(ii) for each group $G_{i} \in \mathcal{G}$ and each block $B_{j} \in \mathcal{B},\left|G_{i} \cap B_{j}\right|=1$, and

(iii) any pair of points from distinct groups occur together either in the hole $Y$ or in exactly one block, but not both.

Here, we list five criteria for determining the existence of ITDs which follow from results of Brouwer and van Rees [10].

Lemma 2.5 Suppose there exists a $T D(k, m)$, a $T D(k, m+1)$, a $T D(k+1, t)$, and $0 \leq u \leq t$. Then there exists an $\operatorname{ITD}(k ; m t+u, u)$.

Lemma 2.6 Suppose there exists a TD(k,m), a TD(k, $m+1)$, a $T D(k, m+$ 2), a TD $(k+2, t), a T D(k, u)$ and $0 \leq u, v \leq t$. Then there exists an $\operatorname{ITD}(k ; m t+u+v, v)$.

Lemma 2.7 Suppose there exists a $T D(k, m)$, a $T D(k, m+1)$, a $T D(k, m+$ 2), a TD $(k+u+1, t)$, a $T D(k+1, m+u)$ and $0 \leq v \leq t-1$. Then there exists an $\operatorname{ITD}(k ; m t+u+v, v)$.

Lemma 2.8 Let $m>1$ and suppose there exist a $T D(k, m)$, a $T D(k, m+1)$ and $a \operatorname{TD}(k+u, t)$. Then there exists an $\operatorname{ITD}(k ; m t+u, m+u)$.

Lemma 2.9 If there exists a $T D(k, m)$, then there exists an $\operatorname{ITD}(k ; m, 1)$. 


\section{Known Designs}

In this section, we collect together previously constructed designs for later use.

$\mathrm{A} \operatorname{PBD}(v,\{k\})$ is usually called a balanced incomplete block design $\operatorname{BIBD}(v, k, 1)$. Resolvable BIBDs are designs that admit a partition of the block set into subsets of blocks that contain every point exactly once. These designs are denoted by $\operatorname{RBIBD}(v, k, 1)$. RBIBDs will be used to construct IPBDs and PBDs. Adjoining a new point to each partition of an $\operatorname{RBIBD}(v, k, 1)$ yields an $\operatorname{IPBD}(v+(v-1) /(k-1),(v-1) /(k-1),\{k+1\})$ and, if $\{k+1,(v-1) /(k-1)\} \subset B(K)$, a $\operatorname{PBD}(v+(v-1) /(k-1), K)$.

$\operatorname{BIBD}(v, 7,1)$ have been extensively studied for a long time. The following result is the culmination of the contributions of several authors, for a survey see, for example, [3].

Lemma 3.1 $A B I B D(v, 7,1)$ exists if $v \equiv 1$ or 7 mod 42 except when $v=43$ and possibly when $v=42 t+1$ and $t \in\{2,3,5,6,12,14,17,19,22,27,33$, $37,39,42,47,59,62\}$ or $v=42 t+7$ and $t \in\{3,19,34,39\}$.

Resolvable BIBDs can be constructed from block disjoint difference families by the Ray-Chaudhuri-Wilson construction, see [24]. Difference families with block size 6 (which can all be made block disjoint) have been investigated by Chen and Zhu [11].

Lemma $3.2([\mathbf{1 1}, \mathbf{2 4}, \mathbf{1 8}])$ Let $q=30 t+1, q \neq 61$ be a prime power. Then there exists a $\operatorname{BIBD}(q, 6,1)$ obtained from a block disjoint difference family, an $\operatorname{RBIBD}(6 q, 6,1)$ and an $\operatorname{IPBD}(216 t+7,36 t+1,\{7\})$.

We also know the following finite set of $\operatorname{RBIBD}(v, 6,1) \mathrm{s}$.

Lemma $3.3([\mathbf{2}, \mathbf{1 6}, \mathbf{1 8}])$ If $4 \leq t \leq 832, t$ is even and $6 t+1$ is a prime power, or if $6 \leq t \leq 296, t$ is even and $5 t+1 \not \equiv 1 \bmod 50$ is a prime power, or if $t \in\{5,57\}$, then an $R B I B D(30 t+6,6,1)$ exists and hence an $I P B D(36 t+7,6 t+1,\{7\})$.

Furthermore, we mention the following group divisible designs.

Lemma $3.4([8,7,3])$ There exist a $7-G D D$ of type $3^{15}$ and 7 -GDDs of type $7^{n}$ for $n \equiv 1 \bmod 6, n \notin\{19,115,145,205,235\}$.

Lemma 3.5 If $v \in B(\{7\})$, then there is a 7-GDD $6^{\frac{v-1}{6}}$.

Proof. Delete just one arbitrary point in a $\operatorname{BIBD}(v, 7,1)$. 
Lemma 3.6 There exist a $\{7,13\}-G D D 6^{51}$ and a $7-G D D 6^{49} 12^{1}$.

Proof. There is a $\operatorname{PBD}(307,\{7,13\})$ with exactly one block of size 13 , see for example [17, Lemma 13.11] or Lemma $4.19(u=7, v=49, w=7, a=6)$. Delete a point not on the 13-block for the GDD of type $6^{51}$ or a point from the 13-block for the GDD of type $6^{49} 12^{1}$.

Lemma 3.7 ([18]) Let $t$ be an integer with $t \notin\{2-6,10-14,16,18-27$, $29-32,34,35,37-40,42-48,51-55,59-62,93-95,98,100-103$, $107-111,116-118,138,139,146,152,154,156-160,163-167,170-174$, $177-181,185-189,191,192,194,195,199,200,201,207-209,215,216$, 219, 221, 228 - 230, 269, 270, 272, 275-278, 283, 285, 286, 326, 334, 339, $342\}$. Then there is a 7-GDD of type $42^{t}$. If $\{7,43\} \subset K$, then there is a $P B D(42 t+1, K)$.

Some useful group divisible designs arise from transversal designs. A proof of the following lemma is given in [23, Lemmas 2.1, 2.2].

Lemma 3.8 If there is a TD $(k, n)$, then there is a $\{k, n\}$-GDD of type $(k-$ $1)^{n}(n-1)^{1}$. If there is a TD(k,n-1), then there is a $\{k, n\}-G D D$ of type $(k-1)^{n-1}(n-1)^{1}$.

Corollary 3.9 There exist a $\{7,13\}-G D D 6^{13} 12^{1}$ and a $\{7,13\}-G D D 6^{12} 12^{1}$. There exist a 7-GDD $6^{49} 48^{1}$ and a 7-GDD $6^{48} 48^{1}$.

Proof. The first two GDDs are obtained from a $\operatorname{TD}(7,13)$ and a $\operatorname{TD}(7,12)$, respectively. The next two GDDs are obtained from a $\operatorname{TD}(7,49)$ and a $\operatorname{TD}(7,48)$, respectively. Note that blocks of size 49 are replaced by blocks of a $\operatorname{BIBD}(49,7,1)$.

The following is a result of Greig [17, Thm. 8.4] which is based on a construction of Brouwer using Baer subplanes.

Lemma 3.10 If $q$ is a prime power, and $0<t<q^{2}-q+1$, then it follows that $t\left(q^{2}+q+1\right)+q^{2}-q+1-t \in B\left(\left\{t+1, q+t,\left(q^{2}-q+1-t\right)^{*}\right\}\right)$ and that there exists an $\operatorname{IPBD}\left(t\left(q^{2}+q+1\right)+q^{2}-q+1-t, q^{2}-q+1-t,\{t+1, q+t\}\right)$.

\section{Constructions}

From GDDs, we construct IPBDs by filling in the groups by appropriate ingredient IPBDs. 
Lemma 4.1 Suppose there is a K-GDD of type $g_{1}, g_{2}, \ldots, g_{n}$. If we have an $I P B D\left(g_{i}+f, f, K\right)$ for $2 \leq i \leq n$, and we have a $P B D\left(g_{1}+f, K\right)$, then there is a $P B D(G+f, K)$ (where $G=\sum_{i=1}^{i=n} g_{i}$ ) containing a flat of order $g_{1}+f$.

In the case our GDD is a TD, we get the following specialization of Lemma 4.1.

Lemma 4.2 Suppose there is a TD(k,n). If $\{k, n\} \subset B(K)$, then there is a $P B D(k n, K)$ containing flats of order $k$ and $n$. If $\{k, n+1\} \subset B(K)$, then there is a $P B D(k n+1, K)$ containing flats of order $k$ and $n+1$.

The second construction is a simple application of Wilson's Fundamental Construction.

Lemma 4.3 Suppose there is a $T D(t, m)$ and a $k$-GDD of type $a^{t}$. If $\{k, a m\} \subset B(K)$, then there is a $P B D(a t m, K)$ containing flats of order $k$ and am. If $\{k, a m+1\} \subset B(K)$, then there is a $P B D(a t m+1, K)$ containing flats of order $k$ and am +1 .

Proof. Take the TD $(t, m)$, a $t$-GDD $m^{t}$ as the master design in Wilson's Fundamental Construction, apply weight $a$ to all points and replace the blocks of size $t$ by the $k$-GDD of type $a^{t}$. In the first case use a $\operatorname{PBD}(k, K)$ and a $\operatorname{PBD}(a m, K)$ to replace the blocks of size $k$ and to fill the groups of size $a m$. In the second case adjoin a common point to all groups and fill these groups with a $\operatorname{PBD}(a m+1, K)$.

The next constructions (Lemmas 4.4, 4.5, 4.6 and 4.10) are taken from [23]. They are applications of Wilson's Fundamental Construction together with filling in the groups by appropriate designs.

Lemma 4.4 Suppose $\{k, n, m(k-1)+1, m(n-1)+1, t(k-1)+1\} \subset B(K)$, a TD(k,n), a TD $(k, n-1)$, and a TD(n+1,m) exists, and $0 \leq t \leq m$. Then there is a $\operatorname{PBD}((n-1) m k+t(k-1)+1, K)$ containing flats of order $k, n, m(k-1)+1, m(n-1)+1$ and $t(k-1)+1$.

Corollary 4.5 Suppose there is a $T D(14, m)$ and $0 \leq t \leq m$. If $\{7,13,6 m+$ $1,12 m+1,6 t+1\} \subset B(K)$, then there is a $P B D(84 m+6 t+1, K)$ containing flats of order $7,13,6 m+1,12 m+1$ and $6 t+1$.

Lemma 4.6 Suppose there exist $K-G D D$ s of group types $1^{n+1}$ and $1^{n} c^{1}$. If there is a $T D(n+1, m)$ and $0 \leq t \leq m$, then there exists a $K-G D D$ of group type $m^{n}(t(c-1)+m)^{1}$.

Corollary 4.7 Suppose $\{u, m, 6 t+m\} \subset B(K)$ and a $T D(u, m)$ exists. If there is a $P B D(u+6, K)$ with a flat of order 7 , and $0 \leq t \leq m$, then there exists a $P B D(m u+6 t, K)$ with flats of order $m$ and $6 t+m$. 
Proof. Take $n=u-1$ and $c=7$ in Lemma 4.6, noting that a $\operatorname{PBD}(u, K)$ can be considered as a $K$-GDD $1^{u}$ and a $\operatorname{PBD}(u+6, K)$ containing a flat of order 7 can be considered as a $K$-GDD $1^{u-1} 7^{1}$. Then fill in groups.

Corollary 4.8 Suppose $u, m, 12 t+m \in B(K)$ and a $T D(u, m)$ exists. If there is a $P B D(u+12, K)$ with a flat of order 13 , and $0 \leq t \leq m$, then there exists a $P B D(m u+12 t, K)$ with flats of order $m$ and $12 t+m$.

Proof. Take $n=u-1$ and $c=13$ in Lemma 4.6.

Lemma 4.9 Suppose $\{u, m, 6 t+m\} \subset B(K)$ and a $T D(u, m)$ exists. If there is a $P B D(u+6, K)$ with a flat of order 7 , a $P B D(u+12, K)$ with a flat of order 13 , and $0 \leq t \leq 2 m$, then there exists a $P B D(m u+6 t, K)$ with flats of order $m$ and $6 t+m$.

Proof. Write $t=x+2 y$ with $x+y \leq m$. Give all points in the $\operatorname{TD}(u, m)$ weight 1 , except for $x$ and $y$ points in the last group, which get weight 7 and 13, respectively. Apply Wilson's Fundamental Construction using $K$-GDD $1^{u}, K$-GDD $1^{u-1} 7^{1}$ and $K$-GDD $1^{u-1} 13^{1}$ derived from given PBDs. Then fill in groups.

Lemma 4.10 Suppose $\{n, m(n-1)+1\} \subset B(K)$, a TD(n,n), and a $T D(n, m)$ exists. Then there is a $\operatorname{PBD}\left(\left(n^{2}-1\right) m+1, K\right)$ containing flats of order $n$ and $m(n-1)+1$.

In the following we prove a few further applications of Wilson's Fundamental Construction which are useful for our purposes.

Lemma 4.11 Let $\{7,13\} \subset B(K)$, suppose there is a $T D(14, m)$ and $0 \leq$ $t \leq m$. If there exist an $\operatorname{IPBD}(6 m+f, f, K)$, and

a) an $\operatorname{IPBD}(12 m+f, f, K)$ and $6 t+f \in B(K)$, or

b) an $\operatorname{IPBD}(6 t+f, f, K)$ and $12 m+f \in B(K)$, or

c) an $\operatorname{IPBD}(12 m+f, f, K)$, an $\operatorname{IPBD}(6 t+f, f, K)$ and $6 m+f \in B(K)$, then there is a $\operatorname{PBD}(84 m+6 t+f, K)$ containing flats of order 7,13 and a flat of order a) $6 t+f$, or b) $12 m+f$, or c) $6 m+f$.

Proof. Truncate all but $t$ points of the last group of the $\operatorname{TD}(14, m)$ to obtain a $\{13,14\}$-GDD $m^{13} t^{1}$. Giving weight 12 to all points of one untruncated group and weight 6 to all other points Wilson's Fundamental Construction yields a $\{7,13\}$-GDD $(6 m)^{12}(12 m)^{1}(6 t)^{1}$ where we use as ingredient GDDs a $\{7,13\}$-GDD $6^{12} 12^{1}$ and a $\{7,13\}$-GDD $6^{13} 12^{1}$ from Corollary 3.9. Now applying Lemma 4.1 completes the proof. 
Lemma 4.12 Let $\{7,13\} \subset B(K)$ and suppose there is a $T D(14, m)$. If there exist an $\operatorname{IPBD}(6(m-1)+f, f, K)$

a) and $12 m+f \in B(K)$, or

b) an $\operatorname{IPBD}(12 m+f, f, K)$ and $6(m-1)+f \in B(K)$,

then there is a $P B D(90 m-78+f, K)$ containing flats of order 7,13 and a flat of order a) $12 m+f$, or b) $6(m-1)+f$.

Proof. Truncate all but one point of a block of the $\operatorname{TD}(14, m)$ to obtain a $\{13,14\}$-GDD $m^{1}(m-1)^{13}$. Giving weight 12 to all points of the group of size $m$ and weight 6 to all other points Wilson's Fundamental Construction yields a $\{7,13\}$-GDD $(12 m)^{1}(6(m-1))^{13}$ where we use as ingredient GDDs a $\{7,13\}$-GDD $6^{12} 12^{1}$ and a $\{7,13\}$-GDD $6^{13} 12^{1}$ from Corollary 3.9. Again, applying Lemma 4.1 completes the proof.

Lemma 4.13 Let $\{7,13\} \subset B(K)$, suppose there is a $T D(t+1, m)$, assume $\{6 t+1,6 t+7\} \subset B(\{7\})$ and $0 \leq r \leq m$. If there exist an $\operatorname{IPBD}(6 m+f, f, K)$,

a) and $6 r+f \in B(K)$, or

b) an $\operatorname{IPBD}(6 r+f, f, K)$ and $6 m+f \in B(K)$,

then there exists a $P B D(6 m t+6 r+f, K)$ containing flats of order 7 and a) $6 r+f$ or b) $6 m+f$.

Proof. Truncate one group of the TD to size $r$ to obtain a $\{t, t+1\}$ GDD $m^{t} r^{1}$. Give weight 6 to all points and apply Wilson's Fundamental Construction with ingredient GDDs 7-GDD $6^{t}$ and 7-GDD $6^{t+1}$ (Lemma 3.5) to get a 7-GDD $(6 m)^{t}(6 r)^{1}$. Apply Lemma 4.1 for the desired PBD.

Lemma 4.14 Let $7 \in B(K)$, suppose there is a $T D(50, m)$ and $0 \leq x, y \leq$ $m$. If there exist an $\operatorname{IPBD}(6 m+f, f, K)$, and

a) an $\operatorname{IPBD}(6 x+f, f, K)$ and a $P B D(6 m+42 y+f, K)$, or

b) an $\operatorname{IPBD}(6 m+42 y+f, f, K)$ and a $P B D(6 x+f, K)$,

then there exists a $P B D(294 m+6 x+42 y+f, K)$.

Proof. Truncate one group of the $\operatorname{TD}(50, m)$ to obtain a $\{49,50\}$-GDD $m^{49} x^{1}$. Giving weight 48 to $y$ points of one untruncated group and weight 6 to all other points Wilson's Fundamental Construction yields a 7-GDD $(6 m)^{48}(6 x)^{1}(6 m+42 y)^{1}$ where we use as ingredient GDDs a 7-GDD $6^{49}$, a 7-GDD $6^{50}$ (from Lemma 3.5), a 7-GDD $6^{48} 48^{1}$ and a 7-GDD $6^{49} 48^{1}$ (from Corollary 3.9). Applying Lemma 4.1 completes the proof. 
Lemma 4.15 Let $7 \in B(K)$, suppose there is a $T D(50, m)$ and $0 \leq x \leq 1$, $0 \leq y \leq 3,0 \leq z \leq m-x-y$. If there exist an $\operatorname{IPBD}(6 m+f, f, K)$ and $a$ $P B D(6 x+12 y+48 z+f, f, K)$, then there exists a $P B D(294 m+6 x+12 y+$ $48 z+f, K)$.

Proof. Truncate one group to size $x+y+z$ and give weight 48 to $z$, weight 12 to $y$ and weight 6 to $x$ points of the truncated group. All remaining points get weight 6 . Now, Wilson's Fundamental Construction yields a 7GDD $(6 m)^{49}(6 x+12 y+48 z)^{1}$ where we use as ingredient GDDs a 7-GDD $6^{49}$, a 7-GDD $6^{50}$ (from Lemma 3.5), a 7-GDD $6^{49} 12^{1}$ (from Lemma 3.6) and a 7-GDD $6^{49} 48^{1}$ (from Corollary 3.9). Again, applying Lemma 4.1 completes the proof.

Lemma 4.16 Let $\{7,13\} \subset B(K)$ and suppose there is a $T D(n, m)$ with $51 \leq n$. There is a $P B D(v, K)$ with

a) $v=294 m+6 t+6 r+1$ if $0 \leq r \leq t \leq m$ and $\{6 m+1,6 t+1,6 r+1\} \subset$ $B(K)$, containing flats of order $7,6 m+1,6 t+1,6 r+1$ and if $r>0$ a flat of order 13;

b) $v=294(m-1)+6 t+6 r+1$ if $0 \leq r<m, 49 \leq t<n,\{6 m+1,6 r+1\} \subset$ $B(K)$ and $6 t+1 \in B(\{7\})$, containing flats of order $7,6 m+1,6 r+1$ and if $r>0, t>49$ a flat of order 13 ;

c) $v=294(m-1)+6 t+6 r-5$ if $1 \leq r \leq m, 50 \leq t \leq n,\{6 m+1,6 r+1\} \subset$ $B(K)$ and $6 t+1 \in B(\{7\})$, containing flats of order $7,6 m+1,6 r+1$ and if $r>1, t>50$ a flat of order 13 ;

d) $v=294(m-2)+6 t+6 r+1$ if $49 \leq r \leq t \leq n, 6 m+1 \in B(K)$ and $\{6 t+1,6 r+1\} \subset B(\{7\})$, containing flats of order $7,6 m+1$ and if $r>49$ a flat of order 13 ;

e) $v=294(m-2)+6 t+6 r-5$ if $50 \leq r \leq t \leq n, 6 m+1 \in B(K)$ and $\{6 t+1,6 r+1\} \subset B(\{7\})$, containing flats of order $7,6 m+1$ and if $r>50$ a flat of order 13 ;

f) $v=300(m-1)+6 t+6 r+1$ if $0 \leq r<50,0 \leq t<m,\{6 m+$ $1,6 m-5,6 t+1\} \subset B(K)$ and $6 r+1 \in B(\{7\})$, containing flats of order 7, $6 m-5,6 t+1$, and if $t>0$ a flat of order 13 , and if $r>0$ a flat of order $6 m+1$;

g) $v=300(m-1)+6 t+6 r-5$ if $1 \leq r<51,1 \leq t \leq m,\{6 m+$ $1,6 m-5,6 t+1\} \subset B(K)$ and $6 r+1 \in B(\{7\})$, containing flats of order $7,13,6 m-5,6 t+1$ and if $r>1$ a flat of order $6 m+1$; 
h) $v=300(m-2)+6 t+6 r+1$ if $0 \leq r<50 \leq t \leq n,\{6 m+1,6 m-5\} \subset$ $B(K)$ and $\{6 t+1,6 r+1\} \subset B(\{7\})$, containing flats of order $7,6 m-5$, and if $r>0, t>50$ a flat of order 13 , and if $r>0$ a flat of order $6 m+1$;

i) $v=300(m-2)+6 t+6 r-5$ if $1 \leq r<51 \leq t \leq n,\{6 m+1,6 m-$ $5\} \subset B(K)$ and $\{6 t+1,6 r+1\} \subset B(\{7\})$, containing flats of order $7,13,6 m-5$ and if $r>1$ a flat of order $6 m+1$.

Proof. First note that a $\operatorname{TD}(49, m)$, $\operatorname{TD}(50, m)$ or $\operatorname{TD}(51, m)$ is embedded in the $\operatorname{TD}(n, m)$. So, for a) truncate two groups of a $\operatorname{TD}(51, m)$ to get a $\{49,50,51\}$-GDD $m^{49} t^{1} r^{1}$. Give weight 6 to all points in Wilson's Fundamental Construction and use the following ingredient GDDs, a 7-GDD $6^{49}$, a 7-GDD $6^{50}$ from Lemma 3.5 and a $\{7,13\}$-GDD $6^{51}$ from Lemma 3.6, to obtain a $\{7,13\}$-GDD $(6 m)^{49}(6 t)^{1}(6 r)^{1}$. Now adjoining an infinite point and filling groups gives the desired PBD. Note that the existence of a flat of order 13 is guaranteed only if there is a block of size 51 in the master GDD, i.e. only if $r, t>0$.

The construction in the other cases is similar (always weight 6 for the points) so that we only need to find the master GDD in each case. Ingredient GDDs 7-GDD $6^{t}$ and 7-GDD $6^{r}$ exist by Lemma 3.5 if required.

For $\mathrm{b} / \mathrm{c})$ spike(=extend) one line of a $\operatorname{TD}(50, m)$ to size $t$, then truncate a group to size $r$. If the $t$ line and $r$ group intersect in a deleted point we get b) a $\{49,50,51, t\}$-GDD $m^{49} r^{1} 1^{t-49}$, otherwise c) a $\{49,50,51, t\}$-GDD $m^{49} r^{1} 1^{t-50}$.

For $\mathrm{d} / \mathrm{e})$ we spike two lines of a $\operatorname{TD}(49, m)$ to size $t$ or $r$, respectively. Here we can assume the groups on the spiked lines coincide as much as possible. There are slight differences if spiked lines intersect within the TD or on the spikes. Within the TD we get d) a $\{49,50,51, r, t\}$-GDD $m^{49} 2^{r-49} 1^{t-r}$, while on the spikes e) a $\{49,50,51, r, t\}$-GDD $m^{49} 2^{r-50} 1^{t-r+1}$ is obtained.

For types $\mathrm{f} / \mathrm{g})$, we truncate a group of a $\operatorname{TD}(51, m)$ to size $t$. Then we truncate a block to size $r$ where we do not delete points from the truncated group. If we truncate a 50-block we get f) a $\{49,50,51, r\}$-GDD $m^{r}(m-$ $1)^{50-r} t^{1}$ (so we need $t<m$ for a group truncation). Truncating a 51-block gives g) a $\{49,50,51, r\}$-GDD $m^{r-1}(m-1)^{51-r} t^{1}$ (so we need $0<t$ ).

For types $\mathrm{h} / \mathrm{i})$, we spike one line of a $\operatorname{TD}(50, m)$ to size $t$. Then we truncate a block to size $r$ of the spiked TD where we do not delete points from the spike. Again, there are slight differences if we truncate a 50-block to obtain g) a $\{49,50,51, r, t\}$-GDD $m^{r}(m-1)^{50-r} 1^{t-50}$ or a 51-block to get i) a $\{49,50,51, r, t\}$-GDD $m^{r-1}(m-1)^{51-r} 1^{t-50}$ (so we need $51 \leq t$ for a spike).

If the master GDDs above do not have groups of order 7 or 13 we can successfully extend Lemma 4.16 by applying Lemma 4.1 on the master GDDs. 
Lemma 4.17 Let $\{7,13\} \subset B(K)$ and suppose there is a $T D(n, m)$ with $51 \leq n$.

a) If $0 \leq r \leq t \leq m$, there is a $P B D(6 m+f, K)$ containing a flat of order $f$ and there are a $P B D(6 t+f, K)$ and a $P B D(6 r+f, K)$ of which at least one PBD contains a flat of order $f$, then a $P B D(294 m+6 t+6 r+f, K)$ exists.

f) If $0 \leq r<50,0 \leq t<m$, there are $P B D(6 m+f, K)$ and $P B D(6 m-6+$ $f, K)$ containing flats of order $f, 6 t+f \in B(K)$ and $6 r+1 \in B(\{7\})$, then a $P B D(300(m-1)+6 t+6 r+f, K)$ exists.

g) If $1 \leq r<51,1 \leq t \leq m$, there are $P B D(6 m+f, K)$ and $P B D(6 m-6+$ $f, K)$ containing flats of order $f, 6 t+f \in B(K)$ and $6 r+1 \in B(\{7\})$, then a $P B D(300(m-1)+6 t+6 r-6+f, K)$ exists.

Lemma 4.18 Let $\{7,13\} \subset B(K)$ and let $q \geq 53$ be a prime power. Suppose there are a $\operatorname{PBD}(6 q+f, K)$ and a $\operatorname{PBD}(6(q-1)+f, K)$ both with a flat of order $f$. Then there exists a $P B D(306 q-6 x+f, K)$ for $0 \leq x \leq 51$. If in addition $q \geq 101$ and there is a $P B D(6(q-2)+f, K)$ with a flat of order $f$, then there exists a $P B D(306 q-6 x-12 y+f, K)$ for $0 \leq x+y \leq 51$.

Proof. Consider an oval (or hyperoval if $q$ is even) in a projective plane $\mathrm{PG}(2, q)$ and delete a point from the (hyper)oval. $\mathrm{A} \mathrm{TD}(q+1, q)$ is obtained in which each group contains at most one point from the (hyper)oval. Deleting groups yields a $\operatorname{TD}(51, q)$ where all groups have exactly one (hyper)oval point. Delete $x$ (hyper)oval points to get a $\{49,50,51\}$-GDD $q^{51-x}(q-1)^{x}$ which in turn with Wilson's Fundamental Construction (weight 6 to all points, 7-GDD $6^{49}, 7$-GDD $6^{50},\{7,13\}$-GDD $\left.6^{51}\right)$ provides a $\{7,13\}$-GDD $(6 q)^{51-x}(6(q-1))^{x}$. Finally, apply Lemma 4.1 to obtain the desired $\operatorname{PBD}(306 q-6 x+f, K)$.

Now delete a non-tangent point (or just a non-hyperoval point if $q$ is even) from $\operatorname{PG}(2, q)$ to obtain a $\operatorname{TD}(q+1, q)$ in which there are exactly $(q+1) / 2$ groups containing no oval point and $(q+1) / 2$ groups containing exactly two oval points (or exactly $q / 2$ groups containing no hyperoval point and $q / 2+1$ groups containing exactly two hyperoval points if $q$ is even). Since we require $q \geq 101$ we are able to delete groups in such a way that we get a $\operatorname{TD}(51, q)$ where all groups have exactly two (hyper)oval points. Now delete from $x$ groups exactly one (hyper)oval point and from $y$ groups two (hyper)oval points for a $\{49,50,51\}$-GDD $q^{51-x-y}(q-1)^{x}(q-2)^{y}$. Again, via Wilson's Fundamental Construction and Lemma 4.1 the desired $\operatorname{PBD}(306 q-6 x-$ $12 y+f, K)$ is obtained.

We also need the singular indirect product construction which we take from [23]. 


\begin{tabular}{llllll}
\hline & $u$ & $v$ & $w$ & $\mathrm{PBD}$ with flat & $a$ \\
\hline 1063 & 7 & 169 & 25 & $\mathrm{TD}(7,24)+\infty$ & 20 \\
1069 & 7 & 169 & 25 & $\mathrm{TD}(7,24)+\infty$ & 19 \\
1567 & 7 & 253 & 37 & $\mathrm{TD}(7,36)+\infty$ & 34 \\
1579 & 7 & 253 & 37 & $\operatorname{TD}(7,36)+\infty$ & 32 \\
\hline
\end{tabular}

Table 2: Proof of Corollary 4.20

Lemma 4.19 If there is an $\operatorname{IPBD}(v, w, K), u \in K, 0 \leq a \leq w \leq v$, the incomplete transversal design $\operatorname{ITD}(u ; v-a, w-a)$ exists, and $u(w-a)+a \in$ $B(K)$, then there is a $P B D(u(v-a)+a, K)$ containing flats of order $u$ and $u(w-a)+a$.

As a first application of the singular indirect product we obtain the following.

Corollary 4.20 1063, 1069, 1567 and 1579 are not essential in a Wilson basis for $N_{1,6}$.

Proof. Use the previous lemma to show that $\{1063,1069,1567,1579\} \subset$ $B(\{7,25,37,55,61,67\})$. The details are given in Table 2 . The needed ITDs are all listed in [5]; they can also be constructed by Lemma 2.5 with $m=16$ or 8 .

In order to apply the constructions above we need as many ingredient designs as possible. So we present in addition to the designs from Section 3 some new (resolvable) BIBDs for $k=6,12,13$ by providing suitable base blocks.

Lemma 4.21 If $834 \leq t \leq 5460, t$ is even and $q=6 t+1$ is a prime power, then an $R B I B D(30 t+6,6,1)$ exists and hence an $\operatorname{IPBD}(36 t+7,6 t+1,\{7\})$.

Proof. As in [16] for $t \leq 832$ we construct $2 t$ base blocks in $G F(5) \times G F(q)$. Let $\omega$ be a generator of the multiplicative group $G F(q)^{*}$ and $d=(q-1) / 2$. We define blocks

$$
S_{0}=\left\{\left(0, \omega^{0}\right),\left(0, \omega^{d}\right),\left(1, \omega^{\gamma_{1}}\right),\left(1, \omega^{\gamma_{1}+d}\right),\left(4, \omega^{\gamma_{2}}\right),\left(4, \omega^{\gamma_{2}+d}\right)\right\}
$$

and

$$
S_{1}=\left\{\left(0, \omega^{3}\right),\left(0, \omega^{3+d}\right),\left(2, \omega^{\gamma_{1}+3}\right),\left(2, \omega^{\gamma_{1}+3+d}\right),\left(3, \omega^{\gamma_{2}+3}\right),\left(3, \omega^{\gamma_{2}+3+d}\right)\right\} .
$$

The base blocks are $S_{b, a}=\left(1, \omega^{6 a}\right) \cdot S_{b}$ with $b=0,1$ and $a=0,1, \ldots, t-1$. It remains to specify $\gamma_{1}, \gamma_{2}$ such that the pure and mixed differences are 
evenly spread amongst the 6 cyclotomic classes. This is done in the appendix Table 8 . Now with a new point $\infty$ adjoined and a new base block $\{\infty,(i, 0)$ : $i=0,1, \ldots, 4\}$ an $\operatorname{RBIBD}(30 t+6,6,1)$ is obtained where a resolution set is formed by the partial development of the base blocks $S_{b, a} \bmod (5,-)$ augmented with the new base block. The development of this resolution set $\bmod (-, q)$ generates the other resolution sets.

The necessary conditions for the existence of a $\operatorname{BIBD}(v, 13,1)$ are $v \equiv 1$ or $13 \bmod 156$. Until now, only a few $\operatorname{BIBD}(v, 13,1)$ with $v$ reasonably small were known to exist. The following BIBDs of order $q, q \equiv 1 \bmod 156$ a prime power, are constructed by difference families using an approach described in [15].

Lemma 4.22 $\operatorname{BIBD}(q, 13,1)$ exist for $q \in A=\{6241,8737,9829,14197$, 15601, 16069, 16381, 16693, 18097, 19813, 20593, 20749, 21061, 21529, 21841, 21997, 22153, 22621, 22777, 23557, 23869, 24181, 24337, 25117, 25741, 26053, 26209, 26833, 27457, 28081, 28549, 29017, 29173, 29641, 30109, 30577, 31357, $31513,31981\}$.

Proof. We construct base blocks in $G F(q)$. Let $w$ be a cube root of unity and let $\left\{m, c, c^{\prime}, c^{\prime \prime}\right\} \subset G F(q)$. We define

$$
S=\left\{0,1, w, w^{2}, c, c w, c w^{2}, c^{\prime}, c^{\prime} w, c^{\prime} w^{2}, c^{\prime \prime}, c^{\prime \prime} w, c^{\prime \prime} w^{2}\right\}
$$

and $S_{i}=m^{i} S$ with $i=0,1, \ldots,(q-1) / 156$. With the values for $m, w, c, c^{\prime}, c^{\prime \prime}$ provided in the appendix Table 9 it is easy to check that every distance is covered exactly once by the blocks $S_{i}$. Note that if $q=p^{n}$ is a proper prime power, then an element $x \in G F(q), x=\sum_{i=0}^{n-1} a_{i} \omega^{i}$ is represented as $x=\sum_{i=0}^{n-1} a_{i} p^{i}$ where $\omega$ is a root of the primitive polynomial for $q$ from Table 7 .

We can make the above difference families block disjoint by using Abel's adder.

Lemma 4.23 (Abel [1]) Suppose $q=k(k-1) t+1$ is a prime power and $a(q, k, 1)$ difference family over $G F(q)$ is given by $B_{i}=m^{i} B_{0}$ for $i=0,1, \ldots, t-1$. Then there is a block disjoint $(q, k, 1)$ difference family over $G F(q)$.

Proof. Define a new difference family by $C_{i}=B_{i}+\mathrm{cm}^{i}$. We will now show there is a choice of $c$ such that $C_{i}$ for $i=0,1, \ldots, t-1$ is the block disjoint difference family. Let $B_{0}=\left(b_{1}, b_{2}, \ldots, b_{k}\right)$. Now $C_{i}=m^{i}\left(B_{0}+c\right)$. Clearly, we cannot have $0 \in\left(B_{0}+c\right)$, so this prohibits $k$ values of $c$. If $C_{i}$ and $C_{j}$ have a common element and $i>j$, then $m^{i} b_{y}+c m^{i}=m^{i} b_{z}+c m^{j}$ for some $y \neq z$, i.e., $c=\left(b_{z}-m^{i-j} b_{y}\right)\left(m^{i-j}-1\right)^{-1}$. This eliminates at most a further 
$k(k-1)$ values of $c$ for each value of $i-j$. So we have prohibited at most $q-(k-1)^{2}$ values of $c$, and there are at least $(k-1)^{2}$ values that work.

So we may again apply the Ray-Chaudhuri-Wilson construction to get RBIBDs.

Lemma 4.24 ([24]) Let the set $A$ be defined as in Lemma 4.22. If $q \in A$, then there is an $\operatorname{RBIBD}(13 q, 13,1)$.

The following family of 13 -GDDs is particularly important as it gives the smallest PBDs in each fibre 19,25,31 or $37 \bmod 42$.

Lemma 4.25 If $t=1,10,13,14,19,23,30,33,44,50,51,55,59,61,63$ or $69 \leq t \leq 2730$, and $q=12 t+1$ is a prime power, then a 13-GDD $13^{q}$ exists and hence a $P B D(156 t+13,\{13\})$.

Proof. For $t=1$ just take a $\operatorname{TD}(13,13)$. For $t=14$ replace the groups of a $\mathrm{TD}(13,169)$ by the $13-\mathrm{GDD} 13^{13}$ just obtained. For $t \neq 1,14$ we construct $t$ base blocks in $G F(13) \times G F(q)$. Let $\omega$ be a generator of the multiplicative group $G F(q)^{*}$. We define a block of size 13

$S=\{(0,0)\} \cup\left\{\left(1, \pm \omega^{0}\right),\left(3, \pm \omega^{\gamma_{1}}\right),\left(4, \pm \omega^{\gamma_{2}}\right),\left(9, \pm \omega^{\gamma_{3}}\right),\left(10, \pm \omega^{\gamma_{4}}\right),\left(12, \pm \omega^{\gamma_{5}}\right)\right\}$.

The base blocks are $S_{a}=\left(1, \omega^{6 a}\right) \cdot S$ with $a=0,1, \ldots, t-1$. With $\gamma_{1}, \ldots, \gamma_{5}$ from the appendix Table 10 we get a 13-GDD $13^{q}$ where the groups are $\{(i, x): i=0,1, \ldots, 12\}$ for $x \in G F(q)$. If we consider the groups to be blocks of size 13 we obtain the desired PBD.

Moreover, we constructed block disjoint difference families of order $q$, $q \equiv 1 \bmod 132$ a prime power, with block size 12 which provide resolvable BIBDs of size 12 when we apply Ray-Chaudhuri-Wilson construction.

Lemma 4.26 $\operatorname{BIBD}(q, 12,1)$ exist for $q \in B=\{5413,6073,6337,6469$, 6733, 6997, 7129, 7393, 7789, 7921, 8053, 8317, 8581, 8713, 9109, 9241, 9769, 9901, 10429, 10957, 11353, 11617, 11881, 12277, 12409, 12541, 13597, 13729, 14389, 14653, 15313, 15973, 16369, 16633, 17029, 17161, 17293, 18217, 18481, 19009, 19141, 19273, 19801, 20593, 20857, 21121, 21517, 21649, 22441, 22573, 23497, 23629, 23761, 23893, 24421, 25609, 25741, 25873, 27061, 27457, 28513, 28909, 29173, 29437, 29569, 29833, 30097, $30493,30757,31153,32077,32341\}$. If $q \in B$, then there is a $\operatorname{BIBD}(q, 12,1)$, an $\operatorname{RBIBD}(12 q, 12,1)$ and an $\operatorname{IPBD}(12 q+(12 q-1) / 11,(12 q-1) / 11,\{13\})$.

Proof. We construct base blocks in $G F(q)$. Let $w$ be a cube root of unity and let $\left\{m, c, c^{\prime}, c^{\prime \prime}\right\} \subset G F(q)$. We define

$$
S=\left\{1, w, w^{2}, c, c w, c w^{2}, c^{\prime}, c^{\prime} w, c^{\prime} w^{2}, c^{\prime \prime}, c^{\prime \prime} w, c^{\prime \prime} w^{2}\right\}
$$


and $S_{i}=m^{i} S$ with $i=0,1, \ldots,(q-1) / 132$. With the values for $m, w, c, c^{\prime}, c^{\prime \prime}$ provided in the appendix Tables 11 and 12 it is easy to check that every distance is covered exactly once by the blocks $S_{i}$.

Lemma 4.27 If $t \notin\{30,33\}, 29 \leq t \leq 2730$, and $q=12 t+1$ is a prime power, then an $\operatorname{RBIBD}(132 t+12,12,1)$ exists and hence an IPBD $(144 t+$ $13,12 t+1,\{13\})$.

Proof. Here, we construct $t$ base blocks in $G F(11) \times G F(q)$. Let $\omega$ be a generator of the multiplicative group $G F(q)^{*}$. We define a block of size 12

$$
S=\left\{\left(0, \pm \omega^{0}\right),\left(1, \pm \omega^{\gamma_{1}}\right),\left(3, \pm \omega^{\gamma_{2}}\right),\left(4, \pm \omega^{\gamma_{3}}\right),\left(5, \pm \omega^{\gamma_{4}}\right),\left(9, \pm \omega^{\gamma_{5}}\right)\right\} .
$$

The base blocks are $S_{a}=\left(1, \omega^{6 a}\right) \cdot S$ with $a=0,1, \ldots, t-1$. With $\gamma_{1}, \ldots, \gamma_{5}$ from the appendix Table 13 and the additional base block $\{\infty,(i, 0): i=$ $0,1, \ldots, 10\}$ an $\operatorname{RBIBD}(132 t+12,12,1)$ is obtained.

We actually tried all prime powers $<32768$ in the appropriate residue class for our direct constructions. In Lemma 4.22, we had failures for $q=$ $156 t+1$ with $q \in\{157,313,625,937,1093,1249,1873,2029,2341,2809$, 3121, 3433, 4057, 4993, 6397, 6553, 6709, 7177, 7333, 7489, 8269, 8581, 8893, 9049, 10141, 10453, 10609, 11701, 12637, 13417, 13729, 14821, 15289, 15913, $17161,17317,18253,19501,24649,28393,32761\}$. Thus we failed in 41 of the 80 possible cases. Greig [15] also looked at cosets of size 13, with only success for $q=21061$ (which adds nothing new here). We will get a quick failure if $\log \left(x^{52 t}-1\right) \equiv 0 \bmod 26$, and we had 5 quick failures. In Lemma 4.26, we had failures for $q=132 t+1$ with $q \in\{397,529,661,1321,1453,1849,2113$, 2377, 3037, 3169, 3301, 3433, 3697, 4093, 4357, 4489, 4621, 5281\}. Thus we failed in 18 of the 90 possible cases. In Lemma 4.27, we had failures for $q=12 t+1$ with $t \in\{1-6,8-10,13-16,19,20,23,24,26,28,30,33\}$. In Lemma 4.25, we had failures for $q=12 t+1$ with $t \in\{2-6,8,9,15,16,20$, $24,26,28,31,34-36,38,45,48,52,56,64\}$.

\section{$5 \quad$ PBD-Closure of $K=\{7,13\}$}

In this section, we show that all positive integers $v \equiv 1 \bmod 6$ are in $B(\{7,13\})$ with the possible exceptions in $Q_{\{7,13\}}$. $Q_{\{7,13\}}$ contains 3960 elements which are listed in the appendix (Table 14). To reduce the problem to a finite one we first construct a representative $\operatorname{PBD}(v,\{7,13\})$ in each possible residue class $r$ modulo $(14 \cdot 84), r \not \equiv 1,7 \bmod 42$.

Lemma 5.1 Let $R=\left\{r_{i, j}: i=0,1, \ldots, 13, j=13,19, \ldots, 79\right\}$ be given by the entries in Table 3. If $r \in R$, then $r \in B(\{7,13\})$. 


\begin{tabular}{rrrrrrrrrr}
\hline 13 & 19 & 25 & 31 & 37 & 55 & 61 & 67 & 73 & 79 \\
\hline 13 & 12955 & 12961 & 21199 & 21205 & 3583 & 14173 & 24763 & 3601 & 3607 \\
2449 & 13039 & 27157 & 21283 & 9529 & 4843 & 18961 & 18967 & 7213 & 18979 \\
3709 & 15475 & 10777 & 21367 & 19021 & 3751 & 16693 & 17875 & 14353 & 13183 \\
2617 & 16735 & 16741 & 19099 & 4993 & 307 & 14425 & 16783 & 15613 & 19147 \\
3877 & 14467 & 6241 & 21535 & 19189 & 8623 & 1573 & 18043 & 14521 & 12175 \\
8665 & 13375 & 21613 & 19267 & 5161 & 8707 & 15769 & 19303 & 13429 & 19315 \\
517 & 14635 & 18169 & 19351 & 17005 & 559 & 14677 & 17035 & 19393 & 19399 \\
601 & 15895 & 19429 & 19435 & 2977 & 4171 & 12409 & 15943 & 19477 & 19483 \\
5389 & 12451 & 19513 & 19519 & 17173 & 8959 & 12493 & 17203 & 7801 & 3103 \\
4297 & 12535 & 16069 & 29011 & 13729 & 4339 & 11401 & 24343 & 21997 & 23179 \\
2029 & 13795 & 2041 & 29095 & 14989 & 2071 & 17365 & 16195 & 7969 & 12679 \\
2113 & 20935 & 16237 & 28003 & 13897 & 5683 & 9217 & 20983 & 6877 & 20995 \\
2197 & 13963 & 15145 & 24559 & 21037 & 6943 & 8125 & 16363 & 21073 & 17551 \\
1105 & 15223 & 16405 & 24643 & 9361 & 1147 & 21145 & 16447 & 4693 & 21163 \\
\hline
\end{tabular}

Table 3: Representative $\operatorname{PBD}\left(r_{i, j},\{7,13\}\right)$ with $r_{i, j} \equiv 84 i+j \bmod 1176$

Proof. We give for each entry from the table a construction and the parameters used. All requisite (I)PBDs are easily obtained from Lemmas 3.1, 3.3, 4.2 or are constructed within this proof. Orders which do not occur in the table but are required as components are marked with an asterisk.

An IPBD of order $3895^{*}$ with a hole of size 649 exists by Lemma 3.2 with $t=18$. The entries 3103, 3607, 12175, 12679, 13183 are from Lemma 3.3 with $t=86,100,338,352,366$. Orders 4993, 7801, 9361, 13729, 14353, 17005, 17551, 19189, 19267 are constructed by Lemma 4.2 with $k=13, n=384$, $600,720,1056,1104,1308,1350,1476,1482$. A PBD of order $3703^{*}$ with a hole of size 517 exists by Corollary 4.5 with $m=43, t=15$. Moreover, 2401*, 2449 are obtained from Lemma 4.10 with $n=7, m=50,51$ and 8707 comes from Lemma 4.11 with $m=97, t=91, f=13$. 4339, 8665 are from Lemma 4.12 with $m=49, f=7$ and $m=97, f=13$. 16447, 28003 are from Lemma 4.15 with $m=49, x=0, y=2, z=42, f=1$ and $m=83$, $x=0, y=3, z=74, f=13$. From Lemma 4.17.a we get 24559, 24643 with $m=83, r=12, t=12,26, f=13$ and 29011, 29095 with $m=97$, $r=12,26, t=68, f=13$. Furthermore, 19303, 19315, 19351, 19393, 19399, 19429, 19435, 19477, 19483, 19513, 19519, 21535, 21613 are from Lemma 4.18 with $q=64, f=1,47 \geq x \geq 11$ or $q=71, f=1, x=32,19$. The entries 6241, 16069, 16693, 21997 are taken from Lemma 4.22. Moreover, 1573, 2041, 2197, 2977, 3601, 4693, 5161, 6877, 7969, 9217, 9529, 10777, 11401, 12493, 12961, 13429, 13897, 14521, 14677, 14989, 15613, 15769, 16237, 17173, 21073, 
27157 are from Lemma 4.25 with $t=10,13,14,19,23,30,33,44,51,59$, $61,69,73,80,83,86,89,93,94,96,100,101,104,110,135,174.7213$ is from Lemma 4.27 with $t=50$. Finally, all remaining orders $r$ can be constructed using Lemma 4.19 (Singular Indirect Product) or Lemma 4.16. The parameters of these constructions are presented in Table 4 and Table 5 . The needed ITDs can all be constructed by Lemmas 2.5-2.9.

Table 4: Applications of the Singular Indirect Product

\begin{tabular}{rrrrrrrrrr}
\hline$r$ & $u$ & $v$ & $w$ & $a$ & $r$ & $u$ & $v$ & $w$ & $a$ \\
\hline 307 & 7 & 49 & 7 & 6 & 517 & 7 & 85 & 13 & 13 \\
559 & 7 & 85 & 7 & 6 & 601 & 7 & 91 & 7 & 6 \\
1105 & 7 & 169 & 13 & 13 & 1147 & 7 & 169 & 7 & 6 \\
$1387^{*}$ & 7 & 223 & 37 & 29 & $1471^{*}$ & 7 & 223 & 37 & 15 \\
$1483^{*}$ & 7 & 217 & 7 & 6 & 2029 & 7 & 295 & 7 & 6 \\
2071 & 7 & 301 & 7 & 6 & 2113 & 7 & 307 & 7 & 6 \\
$2437^{*}$ & 7 & 367 & 61 & 22 & 2617 & 7 & 379 & 7 & 6 \\
3583 & 7 & 517 & 7 & 6 & 3709 & 7 & 583 & 97 & 62 \\
3751 & 13 & 295 & 7 & 7 & 3877 & 7 & 559 & 7 & 6 \\
4171 & 7 & 601 & 7 & 6 & 4297 & 7 & 631 & 91 & 20 \\
4843 & 7 & 727 & 121 & 41 & 5389 & 7 & 871 & 145 & 118 \\
5683 & 7 & 871 & 145 & 69 & 6943 & 7 & 1087 & 181 & 111 \\
8125 & 13 & 637 & 13 & 13 & 8623 & 7 & 1375 & 229 & 167 \\
8959 & 7 & 1447 & 241 & 195 & 12409 & 7 & 1807 & 259 & 40 \\
12451 & 7 & 1813 & 259 & 40 & 12535 & 7 & 1879 & 313 & 103 \\
12955 & 7 & 1951 & 325 & 117 & 13039 & 13 & 1015 & 13 & 13 \\
13375 & 7 & 2023 & 337 & 131 & 13795 & 7 & 2095 & 349 & 145 \\
13963 & 7 & 2065 & 295 & 82 & 14173 & 7 & 2101 & 301 & 89 \\
14425 & 7 & 2143 & 307 & 96 & 14467 & 7 & 2149 & 307 & 96 \\
14635 & 7 & 2239 & 373 & 173 & 15145 & 13 & 1177 & 13 & 13 \\
15223 & 13 & 1183 & 13 & 13 & 15475 & 7 & 2383 & 397 & 201 \\
15895 & 7 & 2455 & 409 & 215 & 15943 & 7 & 2383 & 397 & 123 \\
16195 & 7 & 2359 & 337 & 53 & 16363 & 7 & 2455 & 409 & 137 \\
16405 & 7 & 2395 & 343 & 60 & 16735 & 7 & 2599 & 433 & 243 \\
16741 & 7 & 2401 & 301 & 11 & 16783 & 7 & 2527 & 421 & 151 \\
17035 & 7 & 2437 & 295 & 4 & 17203 & 7 & 2599 & 433 & 165 \\
17365 & 13 & 1513 & 217 & 192 & 17875 & 13 & 1387 & 13 & 13 \\
18043 & 7 & 2743 & 457 & 193 & 18169 & 7 & 2689 & 385 & 109 \\
18967 & 13 & 1471 & 13 & 13 & 20983 & 7 & 3247 & 541 & 291 \\
23179 & 7 & 3463 & 577 & 177 & 24343 & 7 & 3703 & 517 & 263 \\
24763 & 7 & 3895 & 649 & 417 & & & & & \\
\hline & & & & & & & & &
\end{tabular}


Table 5: Applications of Lemma 4.16

\begin{tabular}{ccrrrrcrrrrr}
\hline$r$ & Case & $n$ & $m$ & $t$ & $r$ & $r$ & Case & $n$ & $m$ & $t$ & $r$ \\
\hline 18961 & f) & 65 & 64 & 2 & 8 & 18979 & i) & 65 & 64 & 56 & 8 \\
19021 & c) & 65 & 64 & 56 & 28 & 19099 & i) & 65 & 64 & 56 & 28 \\
19147 & g) & 65 & 64 & 14 & 28 & 20935 & a) & 51 & 71 & 2 & 8 \\
20995 & c) & 72 & 71 & 56 & 14 & 21037 & c) & 72 & 71 & 63 & 14 \\
21145 & a) & 51 & 71 & 2 & 43 & 21163 & i) & 72 & 71 & 63 & 15 \\
21199 & i) & 72 & 71 & 56 & 28 & 21205 & c) & 72 & 71 & 56 & 49 \\
21283 & i) & 72 & 71 & 70 & 28 & 21367 & i) & 72 & 71 & 63 & 49 \\
\hline
\end{tabular}

Lemma 5.2 Let $m \equiv 1 \bmod 14,3501 \leq m \leq 4901$. If $v=12 m+1$, then $v \in B(\{7,13\})$.

Proof. This follows by application of Lemma 4.14 with parameters $m=139$, $x=50, y=20,24, \ldots, 136, f=7 ; m=139, x=99, y=133,137$, $f=7 ; m=167, x=50, y=0,4, \ldots, 164, f=7$ and $m=181, x=$ $50, y=2,6, \ldots, 126, f=7$ which gives $v$ in the intervals $42013-46885$, 47053-47221, 49405-56293 and 53605-58813. The existence of all requisite PBDs is ensured by Lemma 3.1. The remaining values from the interval $47389-49237$ are obtained from Corollary 4.5 with parameters $m=539$, $t=352,408,436,492 ; m=540, t=534 ; m=560, t=86,338,366 ; m=574$, $t=86$; Corollary 4.7 with $u=85, m=547, t=317,401$ and Lemma 4.25 with $t=308$. Here, the requisite PBDs exist by Lemma 3.1, Lemma 5.1, Lemma $4.2(k=7, n=463)$, or Lemma $4.19(u=7, v=421, w=7, a=0$; $u=7, v=463, w=7, a=6 ; u=7, v=931, w=7, a=6)$.

Lemma 5.3 If $v \equiv 1 \bmod 6, v \geq 319825$ then $v \in B(\{7,13\})$.

Proof. Note $v \equiv 1,7 \bmod 42$ is covered by Lemma 3.1. For $v \equiv$ $13,19,25,31,37 \bmod 42$, there is exactly one $r_{i, j}$ from Table 3 such that $v \equiv 7 \cdot 84+r_{i, j} \bmod 1176$. If $v \geq 299628+r_{i, j}$, then there is a unique representation $v=84 m+r_{i, j}$ such that $m \equiv 7 \bmod 14, m \geq 3567$. So a $\operatorname{TD}(14, m)$ exists by Lemma 2.3 and both a $\operatorname{BIBD}(6 m+1,7,1)$ and $\operatorname{BIBD}(12 m+1,7,1)$ exist by Lemma 3.1. Thus, by applying Corollary 4.5 with $r_{i, j}=6 t+1$ we can construct every value $v$ exceeding 319819 except those with $v \equiv r \bmod 1176$ where $r \in E=\{199,235,241,247,283,367,451,535,655,697,955$, $1033\}$. The exceptions occur if $t>m$ that is $r_{i, j}>21403$. In order to apply Corollary 4.5 we need in these cases that $v \geq 15\left(r_{i, j}-1\right)+1$. Noting that $\max \left\{r_{i, j}\right\}=29095$ we can construct in the exceptional cases every value exceeding 435235. 
So it remains to consider the interval $319819<v \leq 435235$ where $v \equiv$ $r \bmod 1176$, with $r \in E$. Write $v=84 m+r_{i, j}$ such that $m \equiv 1 \bmod 14$, $m \geq 3567$. Again a $\operatorname{TD}(14, m)$ exists by Lemma 2.3 , a $\operatorname{BIBD}(6 m+1,7,1)$ exists by Lemma 3.1 and a $\operatorname{PBD}(12 m+1,\{7,13\})$ exists by Lemma 5.2. Hence, if $r_{i, j} \leq 21403$ we can apply Corollary 4.5 to get a $\operatorname{PBD}(v,\{7,13\})$. This works except for $v \equiv 451,955 \bmod 1176$.

Now, let $v \equiv 451,955 \bmod 1176$. There is a representation $v=84 m+$ $r_{4,31}=84 m+21535$ such that $m \equiv 1,7 \bmod 14$. If $v \geq 323011$, then we can construct a $\operatorname{PBD}(v,\{7,13\})$ using Corollary 4.5. We are done if we can give a construction for $v=320323,320827,321499,322003,322675$ : apply Corollary 4.5 with $(m, t)=(3581,3253),(3588,3239),(3574,3547)$, $(3581,3533),(3588,3547)$. The requisite PBDs of order $6 m+1$ are obtained from Lemma 4.18 with $q=71, x=33,40,47, f=1$, those of order $12 m+1$ are obtained from Corollary 4.5 with $m=483, t=386,400,414$ and the one of order $6 t+1$ are constructed in Lemma 5.1.

The next result is obtained with a computer run in which we used all constructions and previously known designs mentioned above to eliminate possible exceptions $v \leq 319819$. There is not enough space to write down all 49369 constructions here, but we provide a web-page

ftp://ftp.math.uni-rostock.de/pub/members/mgruttm/pbdclosure7_13/index.html where for each $v$ at least one construction is given. The computer search left over a set $Q_{\{7,13\}}$ of 3960 possible exceptions listed in Table 14. The largest possible exception is 98683 .

Lemma 5.4 If $v \equiv 1 \bmod 6, v \leq 319819, v \notin Q_{\{7,13\}}$, then $v \in B(\{7,13\})$.

Now, our main result follows from Lemma 5.3 and Lemma 5.4.

Theorem 5.5 If $v \equiv 1 \bmod 6, v \notin Q_{\{7,13\}}$, then $v \in B(\{7,13\})$.

\section{$6 \quad$ PBD-Closure of sets $K$ where $\{7,13\} \subset K \subseteq$ $\{7,13,19,25,31,37,43\}$}

Using the same methods as in the previous section we determined the PBD-closure of all sets $K$ where $\{7,13\} \subset K \subseteq\{7,13,19,25,31,37,43\}$ leaving in each case a number of possible exceptions. The largest exceptions for each $K$ and each fibre $6 t+1$ modulo 42 are represented in Table 6 . Again, there is not enough space for the constructions and the sets of exceptions. Hence, we give details at

ftp://ftp.math.uni-rostock.de/pub/members/mgruttm/pbdclosure7_13/index.html. 
Table 6: Largest possible exception in each residue class $6 t+1$ modulo 42 for all sets $K$ with $\{7,13\} \subseteq K \subseteq$ $\{7,13,19,25,31,37,43\}$

\begin{tabular}{|c|c|c|c|c|c|c|c|}
\hline $\begin{array}{l}6 t+1 \text { modulo } 42 \\
K\end{array}$ & 1 & 7 & 13 & 19 & 25 & 31 & 37 \\
\hline$\{7,13\}$ & 2605 & 1645 & 14293 & 82549 & 98683 & 91507 & 88447 \\
\hline$\{7,13,19\}$ & 2605 & 805 & 13915 & 13081 & 26191 & 90751 & 18811 \\
\hline$\{7,13,25\}$ & 2605 & 1645 & 12277 & 77635 & 12247 & 22417 & 33133 \\
\hline$\{7,13,19,25\}$ & 2605 & 805 & 12193 & 11065 & 12247 & 18763 & 18685 \\
\hline$\{7,13,31\}$ & 2605 & 1645 & 12277 & 18541 & 24721 & 11833 & 48631 \\
\hline$\{7,13,19,31\}$ & 2605 & 805 & 12193 & 11065 & 18463 & 11077 & 18055 \\
\hline$\{7,13,25,31\}$ & 2605 & 1645 & 12277 & 18541 & 11071 & 11833 & 23305 \\
\hline$\{7,13,19,25,31\}$ & 2605 & 805 & 12193 & 11065 & 11071 & 9859 & 17635 \\
\hline$\{7,13,37\}$ & 1975 & 1435 & 13075 & 48739 & 61807 & 64627 & 13099 \\
\hline$\{7,13,19,37\}$ & 1975 & 805 & 13075 & 10099 & 18757 & 36991 & 13099 \\
\hline$\{7,13,25,37\}$ & 1975 & 1435 & 9799 & 23917 & 9811 & 18217 & 10999 \\
\hline$\{7,13,19,25,37\}$ & 1975 & 805 & 8413 & 9511 & 9811 & 17923 & 6631 \\
\hline$\{7,13,31,37\}$ & 1975 & 1435 & 9799 & 18541 & 24385 & 10111 & 10117 \\
\hline$\{7,13,19,3$ & 1975 & 805 & 9799 & 10099 & 18379 & 10111 & 10117 \\
\hline$\{7,13,25,31,37\}$ & 1975 & 1435 & 9799 & 17995 & 7417 & 9817 & 7765 \\
\hline$\{7,13,19,25,31,37\}$ & 1975 & 805 & 5935 & 6613 & 6619 & 5995 & 6631 \\
\hline$\{7,13,43\}$ & 2605 & 1645 & 14293 & 82549 & 95785 & 89575 & 82567 \\
\hline$\{7,13,19,43\}$ & 2605 & 805 & 13915 & 13081 & 26191 & 79159 & 18685 \\
\hline$\{7,13,25,43\}$ & 2605 & 1645 & 12277 & 77635 & 12247 & 22417 & 25447 \\
\hline$\{7,13,19,25,43\}$ & 2605 & 805 & 12193 & 11065 & 12247 & 18763 & 18685 \\
\hline$\{7,13,31,43\}$ & 2605 & 1645 & 12277 & 18541 & 24721 & 11833 & 48631 \\
\hline$\{7,13,19,31,43\}$ & 2605 & 805 & 12193 & 11065 & 18463 & 11077 & 17803 \\
\hline$\{7,13,25,31,4$ & 2605 & 1645 & 12277 & 17281 & 11071 & 11833 & 23305 \\
\hline$\{7,13,19,25,31,43\}$ & 2605 & 805 & 12193 & 11065 & 11071 & 9859 & 17635 \\
\hline$\{7,13,37,43\}$ & 799 & 1435 & 13075 & 48739 & 60379 & 64627 & 13099 \\
\hline$\{7,13,19,37,43\}$ & 799 & 805 & 13075 & 10099 & 18757 & 36991 & 13099 \\
\hline$\{7,13,25,37,43\}$ & 799 & 1435 & 9799 & 23161 & 9811 & 18217 & 10999 \\
\hline$\{7,13,19,25,37,43\}$ & 799 & 805 & 8413 & 9511 & 9811 & 17923 & 6631 \\
\hline$\{7,13,31,37,43\}$ & 799 & 1435 & 9799 & 18541 & 24385 & 10111 & 10117 \\
\hline$\{7,13,19,31,37,43\}$ & 799 & 805 & 9799 & 10099 & 18379 & 9523 & 10117 \\
\hline$\{7,13,25,31,37,43\}$ & 799 & 1435 & 9799 & 17281 & 7417 & 9817 & 7765 \\
\hline$\{7,13,19,25,31,37,43\}$ & 799 & 805 & 5935 & 6613 & 6619 & 5995 & 6631 \\
\hline
\end{tabular}




\section{Acknowledgements}

We wish to thank Charlie Colbourn for providing us the MOLS table in a computer readable format and Julian Abel for many useful comments.

\section{References}

[1] R.J.R. Abel. On the Existence of Balanced Incomplete Block Designs and Transversal Designs. PhD thesis, The University of New South Wales, 1995.

[2] R.J.R. Abel. On the existence of BIBDs with $\lambda=1$ and $6 \leq k \leq 10$. J. Combin. Des., 4:27-50, 1996.

[3] R.J.R. Abel. Some new BIBDs with block size 7. J. Combin. Des., 8:146-150, 2000.

[4] R.J.R. Abel, A.E. Brouwer, C.J. Colbourn, and J.H. Dinitz. Mutually orthogonal Latin squares (MOLS). In C.J. Colbourn and J.H. Dinitz, editors, The CRC Handbook of Combinatorial Designs, pp. 111-142. CRC Press, Boca Raton, 1996. Updates at: http://www.emba.uvm.edu/ dinitz/newresults.html.

[5] R.J.R. Abel, C.J. Colbourn, and J.H. Dinitz. Incomplete MOLS. In C.J. Colbourn and J.H. Dinitz, editors, The CRC Handbook of Combinatorial Designs, pp. 142-172. CRC Press, Boca Raton, 1996. Updates at: http://www.emba.uvm.edu/ dinitz/newresults.html.

[6] R.J.R. Abel, C.J. Colbourn, and M. Wojtas. Concerning seven and eight mutually orthogonal Latin squares. J. Combin. Des., 12:123-131, 2004.

[7] R.J.R. Abel and M. Greig. Balanced incomplete block designs with block size 7. Des. Codes Cryptogr., 13:5-30, 1998.

[8] R.D. Baker. An elliptic semiplane. J. Combin. Theory Ser. A, 25:193195, 1978.

[9] Th. Beth, D. Jungnickel, and H. Lenz. Design Theory. B.I. Wissenschaftsverlag, Bibliographisches Institut, 1985.

[10] A.E. Brouwer and G.H.J. van Rees. More mutually orthogonal Latin squares. Discrete Math., 39:263-281, 1982.

[11] K. Chen and L. Zhu. Existence of $(q, 6,1)$ difference families with $q$ a prime power. Des. Codes Cryptogr., 15:167-173, 1998. 
[12] C.J. Colbourn and J.H. Dinitz, editors. The CRC Handbook of Combinatorial Designs. CRC Press, Boca Raton, 1996.

[13] C.J. Colbourn and J.H. Dinitz. Making the MOLS table. In W.D. Wallis, editor, Computational and Constructive Design Theory, pp. 67134. Kluwer Academic Press, 1996.

[14] B. Du. On finite bases for some PBD-closed sets. J. Combin. Math. Combin. Comput., 20:217-224, 1996.

[15] M. Greig. Some balanced incomplete block design constructions. Congr. Numer., 77:121-134, 1990.

[16] M. Greig. Some group divisible design constructions. J. Combin. Math. Combin. Comput., 27:33-52, 1998.

[17] M. Greig. Designs from projective planes and PBD bases. J. Combin. Des., 7:341-374, 1999.

[18] M. Greig. Recursive constructions of balanced incomplete block designs with block size of 7,8 or 9. Ars Combin., 60:3-54, 2001.

[19] M. Grüttmüller and S. Hartmann. Pan-orientable block designs. In preparation.

[20] H.F. MacNeish. Euler squares. Ann. Math., 23:221-227, 1922.

[21] R.C. Mullin. Finite bases for some pbd-closed sets. Discrete Math., $77: 217-236,1989$

[22] R.C. Mullin and H.-D.O.F. Gronau. PBDs and GDDs. In C.J. Colbourn and J.H. Dinitz, editors, The CRC Handbook of Combinatorial Designs, pp. 185-226. CRC Press, Boca Raton, 1996.

[23] R.C. Mullin and D.R. Stinson. Pairwise balanced designs with blocks sizes $6 t+1$. Graphs Combin., 3:365-377, 1987.

[24] D.K. Ray-Chaudhuri and R.M. Wilson. Existence of resolvable block designs. In J.N. Srivastava et al., editor, Survey of Combinatorial Theory, pp. 361-375. North Holland, Amsterdam, 1973.

[25] R.M. Wilson. An existence theory for pairwise balanced designs I: Composition theorems and morphisms. J. Combin. Theory Ser. A, 13:220$245,1972$.

[26] R.M. Wilson. An existence theory for pairwise balanced designs II: The structure of PBD-closed sets and the existence conjectures. J. Combin. Theory Ser. A, 13:246-273, 1972. 
[27] R.M. Wilson. Construction and uses of pairwise balanced designs. In Combinatorics, volume 55, pp. 18-41. Mathematical Centre Tracts, 1974.

[28] R.M. Wilson. An existence theory for pairwise balanced designs III: Proof of the existence conjectures. J. Combin. Theory Ser. A, 18:7179,1975 .

\section{A Appendix}

The first table gives the primitive polynomials for all proper prime powers 1 mod 12 which are $\leq 2^{15}$. The next six tables give the parameters required in the proof of Lemmas 4.21, 4.22, 4.25. 4.26 (two tables) and 4.27. The entry $*$ indicates that the generating element $\omega$ is a root of the primitive polynomial in the first table. Finally in the last table, we list all integers $v$ for which the existence of a $\operatorname{PBD}(v,\{7,13\})$ is not known.

\begin{tabular}{|c|c|c|c|c|c|c|c|c|c|c|c|}
\hline$p^{n}$ & $p$ & $n$ & $b_{0}, \ldots, b_{n-1}$ & $p^{n}$ & $p$ & $n$ & $b_{0}, \ldots, b_{n-1}$ & $p^{n}$ & $p$ & $n$ & $b_{0}, \ldots, b_{n-1}$ \\
\hline $\begin{array}{r}25 \\
49 \\
14641 \\
28561 \\
529 \\
1369 \\
2209 \\
3721 \\
5329 \\
7921 \\
10609 \\
12769 \\
18769 \\
22801 \\
27889 \\
32761 \\
\end{array}$ & $\begin{array}{r}5 \\
7 \\
11 \\
13 \\
23 \\
37 \\
47 \\
61 \\
73 \\
89 \\
103 \\
113 \\
137 \\
151 \\
167 \\
181\end{array}$ & $\begin{array}{l}2 \\
2 \\
4 \\
4 \\
2 \\
2 \\
2 \\
2 \\
2 \\
2 \\
2 \\
2 \\
2 \\
2 \\
2 \\
2\end{array}$ & $\begin{array}{r}2,1 \\
3,1 \\
8,0,0,1 \\
6,0,1,1 \\
7,1 \\
5,1 \\
13,1 \\
2,1 \\
11,1 \\
6,1 \\
5,1 \\
10,1 \\
6,1 \\
12,1 \\
5,1 \\
18,1\end{array}$ & $\begin{array}{r}625 \\
2401 \\
169 \\
289 \\
841 \\
1681 \\
2809 \\
4489 \\
6241 \\
9409 \\
11449 \\
16129 \\
19321 \\
24649 \\
29929\end{array}$ & $\begin{array}{r}5 \\
7 \\
13 \\
17 \\
29 \\
41 \\
53 \\
67 \\
79 \\
97 \\
107 \\
127 \\
139 \\
157 \\
173\end{array}$ & $\begin{array}{l}4 \\
4 \\
2 \\
2 \\
2 \\
2 \\
2 \\
2 \\
2 \\
2 \\
2 \\
2 \\
2 \\
2 \\
2\end{array}$ & $\begin{array}{r}3,1,0,1 \\
3,0,1,1 \\
2,1 \\
3,1 \\
3,1 \\
12,1 \\
5,1 \\
12,1 \\
3,1 \\
5,1 \\
5,1 \\
3,1 \\
2,1 \\
6,1 \\
5,1\end{array}$ & $\begin{array}{r}15625 \\
121 \\
2197 \\
361 \\
961 \\
1849 \\
3481 \\
5041 \\
6889 \\
10201 \\
11881 \\
17161 \\
22201 \\
26569 \\
32041\end{array}$ & $\begin{array}{r}5 \\
11 \\
13 \\
19 \\
31 \\
43 \\
59 \\
71 \\
83 \\
101 \\
109 \\
131 \\
149 \\
163 \\
179\end{array}$ & $\begin{array}{l}6 \\
2 \\
3 \\
2 \\
2 \\
2 \\
2 \\
2 \\
2 \\
2 \\
2 \\
2 \\
2 \\
2 \\
2\end{array}$ & $\begin{array}{r}2,0,0,0,0,1 \\
7,1 \\
2,0,1 \\
2,1 \\
12,1 \\
3,1 \\
2,1 \\
11,1 \\
2,1 \\
3,1 \\
6,1 \\
14,1 \\
3,1 \\
11,1 \\
7,1\end{array}$ \\
\hline
\end{tabular}

\begin{tabular}{|c|c|c|c|c|c|c|c|c|c|c|c|}
\hline$q$ & $\omega$ & $\gamma_{1}, \gamma_{2}$ & q & $\omega$ & $\gamma_{1}, \gamma_{2}$ & $q$ & $\omega$ & $\gamma_{1}, \gamma_{2}$ & q & $\omega$ & $\gamma_{1}, \gamma_{2}$ \\
\hline 5041 & & 139,2672 & & & & & & & & & \\
\hline $\begin{array}{l}5077 \\
5209\end{array}$ & $\frac{2}{17}$ & $\begin{array}{r}2,4951 \\
202\end{array}$ & $\begin{array}{l}5101 \\
5233\end{array}$ & $\begin{array}{r}6 \\
10\end{array}$ & 4912,4634 & 5113 & 19 & $\begin{array}{l}3542,4393 \\
4898,3922\end{array}$ & 5197 & 7 & 1330,395 \\
\hline $\begin{array}{l}5209 \\
5413\end{array}$ & 17 & $\begin{array}{l}2102,2857 \\
3052,2318\end{array}$ & $\begin{array}{l}5233 \\
5437\end{array}$ & $\begin{array}{r}10 \\
5\end{array}$ & $\begin{array}{r}2552,1021 \\
116,349\end{array}$ & $\begin{array}{l}5281 \\
5449\end{array}$ & $\begin{array}{l}7 \\
7\end{array}$ & $\begin{array}{r}4898,3922 \\
760,4034\end{array}$ & $\begin{array}{l}5329 \\
5521\end{array}$ & 11 & $\begin{array}{l}1184,1846 \\
3692,5446\end{array}$ \\
\hline 5557 & 2 & 1,4127 & 5569 & 13 & 4372,5024 & 5581 & 6 & 586,4997 & 5641 & 14 & 3910,2897 \\
\hline $\begin{array}{l}5653 \\
5749\end{array}$ & $\overline{5}$ & 2926,2486 & $\begin{array}{l}5689 \\
5821\end{array}$ & 11 & $\begin{array}{r}136,4397 \\
3136,1442\end{array}$ & $\begin{array}{l}5701 \\
5857\end{array}$ & 2 & $\begin{array}{r}1,5456 \\
4546,1936\end{array}$ & $\begin{array}{l}5737 \\
5869\end{array}$ & 5 & 1777,1817 \\
\hline 5881 & 31 & 1108,128 & 5953 & $\frac{0}{7}$ & $\begin{array}{l}31304,1842 \\
1364,1807\end{array}$ & $\begin{array}{l}6857 \\
6037\end{array}$ & 5 & $\begin{array}{r}4546,1934 \\
266,3574\end{array}$ & $\begin{array}{l}5869 \\
6073\end{array}$ & 10 & 3622,4907 \\
\hline 6121 & 7 & 124,5162 & 6133 & 5 & 3518,2134 & 6217 & 5 & 1,3365 & $\begin{array}{l}6229 \\
629\end{array}$ & 2 & 1,2813 \\
\hline 41 & $*$ & 320,6106 & 6277 & 2 & 1,662 & 6301 & 10 & 5722,5495 & 6337 & 10 & 418,4532 \\
\hline 361 & 19 & 4796,3673 & 6373 & 2 & 1,3776 & 6397 & 2 & $\begin{array}{r}1,5075 \\
5312\end{array}$ & 6421 & 6 & 760,4985 \\
\hline $\begin{array}{l}6469 \\
6577\end{array}$ & 5 & 974,1021 & $\begin{array}{l}6481 \\
6637 \\
\end{array}$ & $\begin{array}{l}7 \\
2 \\
0\end{array}$ & $\begin{array}{r}2272,1670 \\
2,6337\end{array}$ & $\begin{array}{l}6529 \\
6661\end{array}$ & $\begin{array}{l}7 \\
6\end{array}$ & $\begin{array}{l}5312,4423 \\
5164,6098\end{array}$ & $\begin{array}{l}6553 \\
6673\end{array}$ & $\begin{array}{r}10 \\
5\end{array}$ & $\begin{array}{r}392,6151 \\
1,4238\end{array}$ \\
\hline 6709 & 2 & 2,5383 & 6733 & & 1,32 & 6781 & 2 & 1, 6284 & 6793 & 10 & 2428,782 \\
\hline $\begin{array}{l}6829 \\
6961\end{array}$ & $\begin{array}{r}2 \\
13\end{array}$ & $\begin{array}{r}1,4112 \\
6320,2368\end{array}$ & $\begin{array}{l}6841 \\
6997\end{array}$ & $\begin{array}{r}22 \\
5\end{array}$ & $\begin{array}{l}5764,1853 \\
2500,2939\end{array}$ & $\begin{array}{l}6889 \\
7057\end{array}$ & 5 & $\begin{array}{l}1,3449 \\
1,6080\end{array}$ & $\begin{array}{l}6949 \\
7069\end{array}$ & $\frac{2}{2}$ & $\begin{array}{r}1,6668 \\
6493,6512\end{array}$ \\
\hline $\begin{array}{l}0901 \\
7129\end{array}$ & $\begin{array}{l}10 \\
7\end{array}$ & 4108,533 & 7177 & 10 & 1964,4900 & 7213 & 5 & $\begin{array}{ll}1, & 6080 \\
1 & 1289\end{array}$ & $\begin{array}{r}7069 \\
7237\end{array}$ & 2 & $\begin{array}{r}0493, \\
1,7118\end{array}$ \\
\hline $\begin{array}{l}7297 \\
7369\end{array}$ & $\begin{array}{l}5 \\
7\end{array}$ & $\begin{array}{r}2054,3430 \\
1316,535\end{array}$ & $\begin{array}{l}7309 \\
7393\end{array}$ & $\begin{array}{l}6 \\
5\end{array}$ & $\begin{array}{l}4907,5908 \\
5768,2344\end{array}$ & $\begin{array}{l}7321 \\
7417\end{array}$ & $\begin{array}{l}7 \\
5\end{array}$ & $\begin{array}{l}5764,6206 \\
1490,2236\end{array}$ & $\begin{array}{l}7333 \\
7477\end{array}$ & $\begin{array}{l}6 \\
2\end{array}$ & $\begin{array}{r}3196,7325 \\
1,3056\end{array}$ \\
\hline 7489 & 7 & 1520,2197 & 7537 & 7 & 1706,1486 & $\begin{array}{l}7549 \\
7669\end{array}$ & $\begin{array}{l}0 \\
2 \\
2\end{array}$ & 1,6839 & 7561 & 13 & 6556,4190 \\
\hline $\begin{array}{l}7573 \\
7717\end{array}$ & 2 & $\begin{array}{l}2,6448 \\
2,4930\end{array}$ & $\begin{array}{l}7621 \\
7741\end{array}$ & $\frac{2}{7}$ & $\begin{array}{r}1,5738 \\
1712,3109\end{array}$ & $\begin{array}{l}7669 \\
7753\end{array}$ & $\begin{array}{r}2 \\
10\end{array}$ & $\begin{array}{r}2,1549 \\
1921,6581\end{array}$ & $\begin{array}{l}7681 \\
7789\end{array}$ & $\begin{array}{r}17 \\
2\end{array}$ & $\begin{array}{r}3934,89 \\
1,1610\end{array}$ \\
\hline 7873 & 5 & 1,4520 & 7921 & 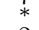 & 1,4049 & 7933 & 2 & 1,6203 & 7993 & 5 & 6080,7828 \\
\hline $\begin{array}{l}8017 \\
8161\end{array}$ & 5 & $\begin{array}{l}1762,5627 \\
6454,1721\end{array}$ & $\begin{array}{l}8053 \\
8209\end{array}$ & $\begin{array}{l}2 \\
7\end{array}$ & $\begin{array}{r}2,7291 \\
4732,7640\end{array}$ & $\begin{array}{l}8089 \\
8221\end{array}$ & $\begin{array}{r}17 \\
2\end{array}$ & $\begin{array}{r}604,2750 \\
2,7999\end{array}$ & $\begin{array}{l}8101 \\
8233\end{array}$ & $\begin{array}{r}6 \\
10\end{array}$ & $\begin{array}{r}3970,32 \\
6032,2938\end{array}$ \\
\hline $\begin{array}{l}8101 \\
8269\end{array}$ & 1 & 0434,4148 & 8293 & 2 & $\begin{array}{r}1,2564 \\
1,2564\end{array}$ & 8317 & 6 & 406,2831 & 8329 & 7 & 4246,5768 \\
\hline 21 & $\begin{array}{r}5 \\
13\end{array}$ & 5282,4522 & $\begin{array}{l}8377 \\
8581\end{array}$ & $\begin{array}{l}5 \\
6 \\
\end{array}$ & $\begin{array}{r}199, \\
7358,7220\end{array}$ & $\begin{array}{l}8389 \\
8629\end{array}$ & 6 & $\begin{array}{l}3154,2429 \\
5146,2231\end{array}$ & 8461 & 6 & $\begin{array}{l}1490,7615 \\
6502,5798\end{array}$ \\
\hline 8677 & 2 & 2,4462 & $\begin{array}{l}801 \\
8689\end{array}$ & 13 & 8086,3332 & 8713 & 5 & $\begin{array}{r}0140,4457 \\
1,457\end{array}$ & 8737 & 5 & 4924,7364 \\
\hline $\begin{array}{l}8761 \\
8941\end{array}$ & $\begin{array}{r}23 \\
6\end{array}$ & $\begin{array}{r}862,8291 \\
934,3845\end{array}$ & $\begin{array}{l}8821 \\
9001\end{array}$ & $\frac{2}{7}$ & $\begin{array}{r}1,7016 \\
550,5153\end{array}$ & $\begin{array}{l}8893 \\
9013\end{array}$ & $\begin{array}{l}5 \\
5\end{array}$ & $\begin{array}{r}1,296 \\
5744,7588\end{array}$ & $\begin{array}{l}8929 \\
9049\end{array}$ & $\begin{array}{r}11 \\
7\end{array}$ & $\begin{array}{l}4030,6302 \\
4762,5168\end{array}$ \\
\hline 9109 & 10 & 1294,5339 & 9133 & 6 & 7312,5465 & 9157 & 6 & 3038,7204 & 9181 & 2 & 2,1078 \\
\hline 9241 & $\begin{array}{r}13 \\
2\end{array}$ & 1724,6877 & & 5 & $\begin{array}{r}1,2627 \\
33324939\end{array}$ & & 2 & 3788,664 & & $\frac{2}{5}$ & $\begin{array}{r}1,2396 \\
86,9103\end{array}$ \\
\hline $\begin{array}{l}9397 \\
9601\end{array}$ & 13 & 4052,8371 & $\begin{array}{l}9409 \\
9613\end{array}$ & 2 & $\begin{array}{r}3332,4939 \\
2,8749\end{array}$ & $\begin{array}{l}9421 \\
9649\end{array}$ & $\frac{2}{7}$ & 5240,7084 & $\begin{array}{l}9433 \\
9661\end{array}$ & $\begin{array}{l}5 \\
2\end{array}$ & $\begin{array}{r}386,9103 \\
1,8888\end{array}$ \\
\hline $\begin{array}{l}9697 \\
9781\end{array}$ & 10 & $\begin{array}{l}2908,911 \\
1306,470\end{array}$ & $\begin{array}{l}9721 \\
9817\end{array}$ & $\begin{array}{l}7 \\
5\end{array}$ & $\begin{array}{r}7846,872 \\
9091 \\
3092\end{array}$ & $\begin{array}{l}9733 \\
9829\end{array}$ & $\begin{array}{r}2 \\
10\end{array}$ & $\begin{array}{r}1,5981 \\
8407,8687\end{array}$ & $\begin{array}{l}9769 \\
9901\end{array}$ & 13 & $\begin{array}{l}1600,6401 \\
2,7246\end{array}$ \\
\hline $\begin{array}{l}9919 \\
9949\end{array}$ & 2 & $\begin{array}{r}1000,3502 \\
2,350\end{array}$ & $\begin{array}{l}3011 \\
9973 \\
\end{array}$ & 11 & 9905,6202 & $\begin{array}{r}10009 \\
\end{array}$ & 11 & $\begin{array}{l}740, \\
7310003\end{array}$ & $\begin{array}{r}5069 \\
10069\end{array}$ & 2 & 1,7385 \\
\hline 10093 & 2 & 1,4121 & 10141 & 2 & 1, 5819 & 10177 & 11 & 7840,7553 & 10201 & & 1,5201 \\
\hline $\begin{array}{l}10273 \\
10369\end{array}$ & $\begin{array}{l}10 \\
13\end{array}$ & $\begin{array}{l}7220,6955 \\
1288,4556\end{array}$ & $\begin{array}{l}10321 \\
10429\end{array}$ & 7 & $\begin{array}{l}6868,9698 \\
5230,4388\end{array}$ & $\begin{array}{l}10335 \\
10453\end{array}$ & 5 & $\begin{array}{r}8,5699 \\
325,2\end{array}$ & 10477 & 2 & $\begin{array}{l}2,8767 \\
2,9613\end{array}$ \\
\hline
\end{tabular}


Table 8: Table of $\gamma_{1}, \gamma_{2}$ for $\operatorname{RBIBD}(5 q+1,6,1)$ construction (cont.)

\begin{tabular}{|c|c|c|c|c|c|c|c|c|c|c|c|}
\hline & & & & & 7 & 597 & & & & $*$ & 3670 \\
\hline $\begin{array}{l}10657 \\
10837\end{array}$ & $\begin{array}{l}7 \\
2\end{array}$ & $\begin{array}{r}832,3224 \\
1,3503\end{array}$ & $\begin{array}{l}10729 \\
10861\end{array}$ & $\begin{array}{l}7 \\
2\end{array}$ & $\begin{array}{r}10004,7402 \\
1,3776\end{array}$ & $\begin{array}{l}10753 \\
10909\end{array}$ & ${ }_{2}^{11}$ & $\begin{array}{r}1526,4051 \\
1,8576\end{array}$ & $\begin{array}{l}10789 \\
10957\end{array}$ & $\frac{2}{5}$ & 1,10187 \\
\hline 10993 & 7 & 8420,7621 & 11113 & 13 & 10982,10129 & 11149 & 10 & 3316,5636 & 11161 & 7 & 550,1244 \\
\hline $\begin{array}{l}11173 \\
11329\end{array}$ & $\begin{array}{l}5 \\
7 \\
\end{array}$ & $\begin{array}{r}1,4388 \\
6988,5372\end{array}$ & $\begin{array}{l}11197 \\
11353\end{array}$ & $\begin{array}{l}2 \\
7\end{array}$ & $\begin{array}{r}2,8641 \\
10726,9839\end{array}$ & $\begin{array}{l}11257 \\
11437\end{array}$ & $\begin{array}{r}10 \\
2\end{array}$ & $\begin{array}{r}3272,3517 \\
1,6539\end{array}$ & $\begin{array}{l}11317 \\
11449\end{array}$ & 2 & $\begin{array}{r}1,10364 \\
1,6374\end{array}$ \\
\hline $\begin{array}{l}11497 \\
11689\end{array}$ & 7 & $\begin{array}{r}830,4138 \\
9446,9679\end{array}$ & $\begin{array}{l}11593 \\
11701\end{array}$ & $\begin{array}{l}5 \\
6\end{array}$ & $\begin{array}{l}6526,1427 \\
2165,7399\end{array}$ & $\begin{array}{l}11617 \\
11821\end{array}$ & $\begin{array}{r}10 \\
2\end{array}$ & $\begin{array}{l}6992,9850 \\
2023,2054\end{array}$ & $\begin{array}{l}11677 \\
11833\end{array}$ & $\begin{array}{l}2 \\
5\end{array}$ & $\begin{array}{r}2,3949 \\
8684,5908\end{array}$ \\
\hline 11881 & 12 & 8360,8950 & 11941 & 10 & 5575,10760 & 11953 & 5 & 1,8366 & 12037 & 5 & 3043,659 \\
\hline $\begin{array}{l}12049 \\
12157\end{array}$ & $\begin{array}{r}13 \\
2\end{array}$ & $\begin{array}{r}10048,4532 \\
1,4187\end{array}$ & $\begin{array}{l}12073 \\
12241\end{array}$ & $\begin{array}{l}7 \\
7\end{array}$ & $\begin{array}{l}1480,3446 \\
1994,9940\end{array}$ & $\begin{array}{l}12097 \\
12253\end{array}$ & $\begin{array}{l}5 \\
2\end{array}$ & $\begin{array}{r}4756,11165 \\
1,4187\end{array}$ & $\begin{array}{l}12109 \\
12277\end{array}$ & $\begin{array}{l}6 \\
2\end{array}$ & $\begin{array}{r}1156,9107 \\
1,10226\end{array}$ \\
\hline $\begin{array}{l}12289 \\
12421\end{array}$ & $\begin{aligned} 11 \\
7\end{aligned}$ & $\begin{array}{r}4172,2470 \\
3604,10133\end{array}$ & $\begin{array}{l}12301 \\
12433\end{array}$ & $\begin{array}{r}2 \\
13\end{array}$ & $\begin{array}{l}1,10916 \\
6362,8014\end{array}$ & $\begin{array}{l}12373 \\
12457\end{array}$ & $\begin{array}{r}2 \\
10\end{array}$ & $\begin{array}{r}1,1193 \\
3400,7106\end{array}$ & $\begin{array}{l}12409 \\
12517\end{array}$ & $\begin{array}{l}7 \\
6\end{array}$ & $\begin{array}{r}7448,10492 \\
2404,5555\end{array}$ \\
\hline $\begin{array}{l}12541 \\
12601\end{array}$ & $\begin{array}{l}14 \\
11\end{array}$ & $\begin{array}{r}4735,8225 \\
10718,2314\end{array}$ & $\begin{array}{l}12553 \\
12613\end{array}$ & $\begin{array}{r}5 \\
2\end{array}$ & $\begin{array}{r}7852,7853 \\
1,12092\end{array}$ & $\begin{array}{l}12577 \\
12637\end{array}$ & $\begin{array}{r}10 \\
2\end{array}$ & $\begin{array}{r}4880,12298 \\
1,1064\end{array}$ & $\begin{array}{l}12589 \\
12697\end{array}$ & $\begin{array}{l}2 \\
7\end{array}$ & $\begin{array}{r}1,10634 \\
2468,2143\end{array}$ \\
\hline 12721 & 13 & 1570,7628 & 12757 & 21 & 2,7567 & 12769 & & 1,8783 & 12781 & 2 & 1,1100 \\
\hline $\begin{array}{l}12829 \\
12973\end{array}$ & $\begin{array}{r}2 \\
14\end{array}$ & $\begin{array}{r}1,662 \\
1,2519\end{array}$ & $\begin{array}{l}12841 \\
13009\end{array}$ & 21 & 0,418 & $\begin{array}{l}12853 \\
13033 \\
\end{array}$ & $\begin{array}{r}5 \\
5 \\
5\end{array}$ & $\begin{array}{r}1,5402 \\
5006,9103\end{array}$ & $\begin{array}{l}12889 \\
13093\end{array}$ & $\begin{array}{r}13 \\
6\end{array}$ & $\begin{array}{l}982,8822 \\
162,5744\end{array}$ \\
\hline $\begin{array}{l}13177 \\
13381\end{array}$ & 10 & $\begin{array}{r}1,3584 \\
7855,4640\end{array}$ & $\begin{array}{l}13249 \\
13417\end{array}$ & $\begin{array}{l}7 \\
5\end{array}$ & $\begin{array}{r}11582,11584 \\
10640,7327\end{array}$ & $\begin{array}{l}13297 \\
1344\end{array}$ & 11 & $\begin{array}{r}12055,10058 \\
8728,4601\end{array}$ & $\begin{array}{l}13309 \\
13477\end{array}$ & $\begin{array}{l}6 \\
2\end{array}$ & $\begin{array}{r}670,4796 \\
2,9088\end{array}$ \\
\hline $\begin{array}{l}13513 \\
13669\end{array}$ & $\begin{array}{l}5 \\
6\end{array}$ & $\begin{array}{r}6772,3707 \\
1,335\end{array}$ & $\begin{array}{l}13537 \\
13681\end{array}$ & $\begin{array}{r}7 \\
22\end{array}$ & $\begin{array}{r}9542,7150 \\
12352,11822\end{array}$ & $\begin{array}{l}13597 \\
13693\end{array}$ & $\begin{array}{l}5 \\
6\end{array}$ & $\begin{array}{l}2756,2170 \\
6652,1856\end{array}$ & $\begin{array}{l}13633 \\
13729\end{array}$ & $\begin{array}{r}5 \\
23\end{array}$ & $\begin{array}{r}12112,12539 \\
13240\end{array}$ \\
\hline $\begin{array}{l}13789 \\
14029\end{array}$ & $\begin{array}{l}7 \\
6\end{array}$ & $\begin{array}{l}00,1728 \\
766,6005\end{array}$ & $\begin{array}{l}13873 \\
14149\end{array}$ & $\begin{array}{r}5 \\
5 \\
6\end{array}$ & $\begin{array}{r}3838,11099 \\
11944,10304\end{array}$ & $\begin{array}{l}13921 \\
14173\end{array}$ & $\begin{array}{l}7 \\
2\end{array}$ & $\begin{array}{r}316,1814 \\
2,3319\end{array}$ & $\begin{array}{l}13933 \\
14197\end{array}$ & 11 & $\begin{array}{r}1,5 \\
3098\end{array}$ \\
\hline $\begin{array}{l}14029 \\
14389\end{array}$ & $\begin{array}{l}0 \\
2 \\
2\end{array}$ & 7951,8267 & $\begin{array}{l}14149 \\
14281 \\
14401\end{array}$ & $\begin{array}{l}19 \\
11\end{array}$ & 12674,4423 & $\begin{array}{l}14173 \\
14293\end{array}$ & 6 & 1468,10805 & $\begin{array}{l}14191 \\
14341\end{array}$ & $\begin{array}{r}1 \\
2 \\
22\end{array}$ & $\begin{array}{l}4,3050 \\
2,3193 \\
3956\end{array}$ \\
\hline $\begin{array}{l}14589 \\
14461\end{array}$ & 2 & 1,14270 & $\begin{array}{l}14401 \\
14533\end{array}$ & ${ }_{2}^{1}$ & 2,1957 & $\begin{array}{l}1443 \\
14557\end{array}$ & $\begin{array}{l}5 \\
2\end{array}$ & $\begin{array}{r}28,1018 \\
2,8212\end{array}$ & $\begin{array}{l}14449 \\
14593\end{array}$ & & 1,10751 \\
\hline $\begin{array}{l}14629 \\
14737\end{array}$ & $\begin{array}{r}2 \\
10\end{array}$ & $\begin{array}{l}13699,1328 \\
1588,13580\end{array}$ & $\begin{array}{l}14641 \\
14797\end{array}$ & * 2 & $\begin{array}{l}1,5909 \\
1,263\end{array}$ & $\begin{array}{l}14653 \\
14821\end{array}$ & $\stackrel{2}{2}$ & $\begin{array}{l}1,10595 \\
2,7120\end{array}$ & $\begin{array}{l}14713 \\
14869\end{array}$ & $\begin{array}{l}5 \\
2 \\
2\end{array}$ & $\begin{array}{r}13996,11606 \\
1,8693\end{array}$ \\
\hline $\begin{array}{l}14929 \\
15121\end{array}$ & ${ }_{11}^{7}$ & $\begin{array}{r}11116,6950 \\
952,953\end{array}$ & $\begin{array}{r}15013 \\
15193 \\
15089\end{array}$ & $\begin{array}{r}2 \\
5\end{array}$ & $\begin{array}{l}72 \\
89\end{array}$ & $\begin{array}{l}15061 \\
15217\end{array}$ & $\begin{array}{r}2 \\
10\end{array}$ & $\begin{array}{r}1,5276 \\
12508,13388\end{array}$ & $\begin{array}{l}15073 \\
15241\end{array}$ & $\begin{array}{r}5 \\
11\end{array}$ & $\begin{array}{r}1,1718 \\
11120,11860\end{array}$ \\
\hline $\begin{array}{l}15277 \\
15361\end{array}$ & $\begin{array}{l}6 \\
7\end{array}$ & $\begin{array}{l}14104,2312 \\
2696,10456\end{array}$ & $\begin{array}{l}15289 \\
15373\end{array}$ & $\begin{array}{r}11 \\
2\end{array}$ & $\begin{array}{l}2423 \\
3191\end{array}$ & $\begin{array}{l}15313 \\
15493\end{array}$ & $\begin{array}{r}5 \\
5\end{array}$ & $\begin{array}{r}13384,3734 \\
682,11783\end{array}$ & $\begin{array}{l}15349 \\
15541\end{array}$ & $\begin{array}{l}2 \\
6\end{array}$ & $\begin{array}{r}1,9620 \\
11810,11059\end{array}$ \\
\hline 15601 & 23 & 14680,1067 & $\begin{array}{l}15625 \\
15817\end{array}$ & $\bar{*}$ & 2966 & $\begin{array}{l}15649 \\
15877\end{array}$ & 11 & 2720,13648 & 15661 & 2 & 1,6683 \\
\hline 15901 & 10 & 7934,15874 & $\begin{array}{l}15813 \\
15913\end{array}$ & 5 & 10399,15884 & $\begin{array}{l}1587 \\
15937\end{array}$ & 7 & 6034, 14057 & $\begin{array}{l}15889 \\
15973\end{array}$ & $2 \frac{1}{7}$ & $\begin{array}{l}1046,11086 \\
6202,15356\end{array}$ \\
\hline $\begin{array}{l}16033 \\
16141\end{array}$ & $\begin{array}{l}5 \\
6\end{array}$ & $\begin{array}{r}11842,12152 \\
8495,2323\end{array}$ & $\begin{array}{l}16057 \\
16189\end{array}$ & $\begin{array}{l}7 \\
2\end{array}$ & 14072,13192 & $\begin{array}{l}16069 \\
16249\end{array}$ & $\begin{array}{r}2 \\
17\end{array}$ & $\begin{array}{r}1,11744 \\
1552,617\end{array}$ & $\begin{array}{l}16129 \\
16273\end{array}$ & 7 & $\begin{array}{l}9344,14698 \\
4628,14185\end{array}$ \\
\hline $\begin{array}{l}16333 \\
16453\end{array}$ & $\begin{array}{l}0 \\
2\end{array}$ & 1,2051 & $\begin{array}{l}16369 \\
16477\end{array}$ & $\begin{array}{l}7 \\
2\end{array}$ & 2384,7294 & $\begin{array}{l}10249 \\
16381 \\
16561\end{array}$ & $\begin{array}{r}17 \\
2 \\
7\end{array}$ & $\begin{array}{l}102,2777 \\
1,267\end{array}$ & $\begin{array}{l}1027 \\
16417 \\
16573\end{array}$ & 10 & 12104,10915 \\
\hline 16633 & 15 & 80,8360 & 16657 & 5 & 3,5075 & $\begin{array}{l}10061 \\
16693\end{array}$ & 2 & 2,11515 & $\begin{array}{l}10573 \\
16729\end{array}$ & 13 & 4898,10468 \\
\hline $\begin{array}{l}16741 \\
17029\end{array}$ & $\begin{array}{r}6 \\
10\end{array}$ & $\begin{array}{r}8080,5459 \\
11056,8372\end{array}$ & $\begin{array}{l}16921 \\
17041\end{array}$ & $\begin{array}{r}17 \\
7\end{array}$ & $\begin{array}{l}12920,14473 \\
11060,14719\end{array}$ & $\begin{array}{l}16981 \\
17053\end{array}$ & $\begin{array}{l}\overline{2} \\
2\end{array}$ & 300 & $\begin{array}{l}16993 \\
17077\end{array}$ & 10 & 1474,11753 \\
\hline 17137 & 5 & 16760,12640 & 17161 & $*$ & 11225 & 17209 & 14 & 12448,4949 & 17257 & 5 & 7484,7450 \\
\hline $\begin{array}{l}17293 \\
17389\end{array}$ & $\begin{array}{l}7 \\
2\end{array}$ & & $\begin{array}{l}17317 \\
17401\end{array}$ & 12 & & $\begin{array}{l}17341 \\
17449\end{array}$ & $\begin{array}{r}6 \\
14\end{array}$ & $\begin{array}{r}11932 \\
15778\end{array}$ & $\begin{array}{l}17377 \\
17497\end{array}$ & $\begin{array}{l}7 \\
5\end{array}$ & $\begin{array}{l}226 \\
302\end{array}$ \\
\hline $\begin{array}{l}17509 \\
17737\end{array}$ & $\begin{array}{l}2 \\
7\end{array}$ & $\begin{array}{l}018 \\
837\end{array}$ & $\begin{array}{l}17569 \\
17749\end{array}$ & 11 & 15652 & $\begin{array}{l}17581 \\
17761\end{array}$ & $\begin{array}{l}10 \\
19\end{array}$ & $\begin{array}{l}3955,10442 \\
17426,2638\end{array}$ & $\begin{array}{l}17713 \\
17881\end{array}$ & $\begin{array}{l}7 \\
7\end{array}$ & $\begin{array}{r}15436,12746 \\
2056,3233\end{array}$ \\
\hline $\begin{array}{l}17929 \\
18049\end{array}$ & $\begin{array}{l}11 \\
13\end{array}$ & $\begin{array}{r}16508,2962 \\
10544,12631\end{array}$ & $\begin{array}{l}17977 \\
18061\end{array}$ & $\begin{array}{l}5 \\
6 \\
6\end{array}$ & $\begin{array}{l}2812,4379 \\
2146,5492\end{array}$ & $\begin{array}{l}17989 \\
18097\end{array}$ & $\begin{array}{r}1 \\
2 \\
5\end{array}$ & $\begin{array}{r}1,998 \\
14114,2890\end{array}$ & $\begin{array}{l}18013 \\
18121\end{array}$ & 23 & 4336,13016 \\
\hline $\begin{array}{l}18043 \\
18229\end{array}$ & $\begin{array}{r}10 \\
5 \\
2\end{array}$ & 1,12989 & $\begin{array}{l}180169 \\
18253\end{array}$ & $1 \frac{0}{5}$ & $\begin{array}{r}3140,6952 \\
3140,6986\end{array}$ & $\begin{array}{l}18181 \\
18289\end{array}$ & $\begin{array}{r}5 \\
2 \\
13\end{array}$ & 1,1661 & $\begin{array}{l}18121 \\
18217 \\
18301\end{array}$ & $\begin{array}{r}23 \\
7\end{array}$ & 14410,15248 \\
\hline $\begin{array}{l}18229 \\
18313\end{array}$ & 10 & $\begin{array}{l}2,4819 \\
84,7108\end{array}$ & $\begin{array}{l}18253 \\
18397\end{array}$ & $\begin{array}{l}5 \\
6\end{array}$ & $\begin{array}{l}12986 \\
, 3569\end{array}$ & $\begin{array}{l}18289 \\
18433\end{array}$ & $\begin{array}{r}13 \\
5\end{array}$ & $\begin{array}{r}14810,6064 \\
6296,826\end{array}$ & $\begin{array}{l}18301 \\
18457\end{array}$ & $\begin{array}{l}6 \\
5\end{array}$ & $\begin{array}{l}1462,8693 \\
9572,6664\end{array}$ \\
\hline 18481 & 13 & $\begin{array}{r}3824,1474 \\
15734,17341\end{array}$ & 18493 & $\frac{2}{2}$ & $\begin{array}{l}10612 \\
10984\end{array}$ & 18517 & 6 & 16918,18227 & 18541 & 6 & 10774,10541 \\
\hline 18769 & 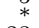 & 1,6425 & 18793 & 5 & 12797 & 18913 & 10 & 9290,10456 & 18973 & 2 & 1,18872 \\
\hline 19009 & 23 & 00,17702 & $\begin{array}{l}19069 \\
19237\end{array}$ & 2 & 13226 & $\begin{array}{l}19081 \\
19249\end{array}$ & 17 & 10144,13082 & 19141 & $\frac{2}{5}$ & $181 \frac{1}{2} 15572$ \\
\hline 19309 & 6 & 3886,2162 & 19321 & & 17155 & 19333 & 2 & 1, 13478 & 19381 & 7 & 5315,5989 \\
\hline $\begin{array}{l}19417 \\
19489\end{array}$ & $\begin{array}{r}5 \\
19\end{array}$ & 676,1226 & $\begin{array}{l}19429 \\
19501\end{array}$ & $\begin{array}{l}6 \\
2\end{array}$ & $\begin{array}{l}0025 \\
8218\end{array}$ & $\begin{array}{l}19441 \\
19597\end{array}$ & 13 & $\therefore 1$ & $\begin{array}{l}19477 \\
19609\end{array}$ & $\begin{array}{r}6 \\
13\end{array}$ & $\begin{array}{r}12616,13475 \\
3680,16945\end{array}$ \\
\hline $\begin{array}{l}19681 \\
19801\end{array}$ & 11 & $\begin{array}{l}7420,1004 \\
844,15794\end{array}$ & $\begin{array}{l}19717 \\
19813\end{array}$ & $\frac{2}{2}$ & 11135 & $\begin{array}{l}19753 \\
19861\end{array}$ & 5 & 1,11984 & $\begin{array}{l}19777 \\
19993\end{array}$ & 11 & $\begin{array}{l}1,6197 \\
902,6298\end{array}$ \\
\hline 20029 & 2 & 1,2834 & 20089 & 7 & 10291 & 20101 & 6 & 3382,13340 & 20113 & 10 & 11512,5825 \\
\hline $\begin{array}{l}20149 \\
20269\end{array}$ & $\stackrel{2}{2}_{2}$ & $\begin{array}{l}09,4421 \\
2,14377\end{array}$ & $\begin{array}{l}20161 \\
20341\end{array}$ & $\begin{array}{r}13 \\
2\end{array}$ &, $\begin{array}{r}4951 \\
11054\end{array}$ & $\begin{array}{l}20173 \\
20353\end{array}$ & $\begin{array}{l}2 \\
5\end{array}$ & $\begin{array}{r}2,13003 \\
15032,11791\end{array}$ & $\begin{array}{l}20233 \\
20389\end{array}$ & $\begin{array}{l}5 \\
6\end{array}$ & $\begin{array}{l}9229 \\
9485\end{array}$ \\
\hline $\begin{array}{l}20509 \\
20641\end{array}$ & $\begin{array}{l}\overline{2} \\
7\end{array}$ & $\begin{array}{r}1 \\
11768,13403 \\
\end{array}$ & $\begin{array}{l}20521 \\
20749\end{array}$ & $\begin{aligned} 11 \\
2\end{aligned}$ & $\begin{array}{r}9176,2035 \\
2,13165\end{array}$ & $\begin{array}{l}20533 \\
20773\end{array}$ & $\begin{array}{l}2 \\
2\end{array}$ & $\begin{array}{r}2,49 \\
2,5089\end{array}$ & $\begin{array}{l}20593 \\
20809\end{array}$ & $\begin{array}{l}5 \\
7\end{array}$ & $\begin{array}{r}13255,11429 \\
9512,12859\end{array}$ \\
\hline $\begin{array}{l}20857 \\
21061\end{array}$ & 10 & $\begin{array}{r}19276,2 \\
15310,13976\end{array}$ & $\begin{array}{l}20929 \\
21121\end{array}$ & $\begin{array}{r}7 \\
19\end{array}$ & $\begin{array}{r}12710,8773 \\
1706,4267\end{array}$ & $\begin{array}{l}21001 \\
21157\end{array}$ & $1 \frac{1}{2}$ & 8192,2038 & 21013 & 2 & 189017255 \\
\hline $\begin{array}{l}21001 \\
21193\end{array}$ & $1 \frac{1}{5}$ & $\begin{array}{l}10,15902 \\
38,15902\end{array}$ & $\begin{array}{l}21127 \\
21487\end{array}$ & 6 & $\begin{array}{l}14201 \\
14924\end{array}$ & $\begin{array}{l}21157 \\
21313 \\
21493\end{array}$ & $\begin{array}{l}2 \\
5 \\
2\end{array}$ & 10700,17416 & $\begin{array}{l}21169 \\
21397\end{array}$ & $\begin{array}{r}13 \\
2 \\
5\end{array}$ & $\begin{array}{r}8290,9988 \\
2,4144\end{array}$ \\
\hline $\begin{array}{l}21433 \\
21529\end{array}$ & 11 & $\begin{array}{l}0272,1958 \\
0156,3029\end{array}$ & $\begin{array}{l}21481 \\
21577\end{array}$ & 13 & 18757,15350 & 214 & 2 & 2, & $\begin{array}{l}21501 \\
21601\end{array}$ & 7 & 4060,20948 \\
\hline $\begin{array}{l}21613 \\
21757\end{array}$ & $\begin{array}{l}2 \\
5\end{array}$ & $\begin{array}{r}1,2357 \\
14581,12776\end{array}$ & $\begin{array}{l}21649 \\
21817\end{array}$ & $\begin{array}{r}14 \\
7\end{array}$ & $\begin{array}{r}19786,5327 \\
12268,10223\end{array}$ & $\begin{array}{l}21661 \\
21841\end{array}$ & $1 \stackrel{2}{1}$ & $\begin{array}{r}1,461 \\
20860,6203\end{array}$ & $\begin{array}{l}21673 \\
21937\end{array}$ & $\begin{array}{r}10 \\
7\end{array}$ & $\begin{array}{l}14456,13774 \\
18436,18515\end{array}$ \\
\hline 21961 & 17 & 1196 & 21997 & 7 & 17150,3709 & 22093 & 6 & $1 \overline{3} 552,17411$ & $\begin{array}{l}22129 \\
22129\end{array}$ & 19 & 4874,18760 \\
\hline $\begin{array}{l}22153 \\
22369\end{array}$ & $\begin{array}{r}5 \\
11\end{array}$ & $\begin{array}{r}3850,1205 \\
1366,10478\end{array}$ & $\begin{array}{l}22189 \\
22381\end{array}$ & $10^{2}$ & 16025,13972 & 22441 & 14 & 1718 & $\begin{array}{l}222753 \\
22453\end{array}$ & $\begin{array}{l}5 \\
5\end{array}$ & 5140,9476 \\
\hline $\begin{array}{l}22501 \\
22669\end{array}$ & $\stackrel{2}{2}$ & $\begin{array}{l}20270 \\
15937\end{array}$ & $\begin{array}{l}22549 \\
22717\end{array}$ & $\stackrel{2}{2}$ & 14 & $\begin{array}{l}22573 \\
22741\end{array}$ & 6 & $\begin{array}{l}11153,15028 \\
16178,16282\end{array}$ & $\begin{array}{l}22621 \\
22777\end{array}$ & $\begin{array}{l}2 \\
7\end{array}$ & $\begin{array}{r}1,7058 \\
15584,12658\end{array}$ \\
\hline 22801 & * & 050 & 22861 & 2 & 148 & 22921 & 7 & 17522,17704 & 22993 & 5 & 18682,12599 \\
\hline 23173 & $\begin{array}{l}5 \\
5 \\
5\end{array}$ & $\begin{array}{l}11090 \\
15995\end{array}$ & 23197 & 2 & $10 /$ & $\begin{array}{l}23041 \\
23209\end{array}$ & 31 & $\begin{array}{l}19196,14560 \\
21868,16094\end{array}$ & $\begin{array}{l}23269 \\
23269\end{array}$ & 6 & 1018,2183 \\
\hline $\begin{array}{l}23293 \\
23557\end{array}$ & 5 & 2196,9883 & $\begin{array}{l}23473 \\
23581\end{array}$ & $\overline{5}$ & $\begin{array}{r}106 \\
228\end{array}$ & $\begin{array}{l}23497 \\
23593\end{array}$ & 5 & $\begin{array}{l}1162,15242 \\
3658,12836\end{array}$ & $\begin{array}{l}23509 \\
23629\end{array}$ & 2 & 24 \\
\hline 23677 & 5 & $\begin{array}{r}9142,4175 \\
9393\end{array}$ & $\begin{array}{l}23689 \\
23857\end{array}$ & $1 \frac{0}{5}$ & $\begin{array}{r}22840,21410 \\
5786,2950\end{array}$ & $\begin{array}{l}23593 \\
23761 \\
23869\end{array}$ & $\begin{array}{l}5 \\
7 \\
2\end{array}$ & $\begin{array}{r}3658,12836 \\
10520,11980 \\
2707\end{array}$ & $\begin{array}{l}23629 \\
23773 \\
23893\end{array}$ & $\begin{array}{l}2 \\
5 \\
5\end{array}$ & $\begin{array}{l}1,1244 \\
15002\end{array}$ \\
\hline $\begin{array}{l}230317 \\
23919\end{array}$ & 2 & $\begin{array}{r}10393,16862 \\
5878\end{array}$ & 23857 & $\begin{array}{r}5 \\
7\end{array}$ & $\begin{array}{r}1072,19442 \\
12862,22823\end{array}$ & $\begin{array}{l}23869 \\
23977 \\
\end{array}$ & $\frac{2}{5}$ & 2701,13469 & 24001 & 14 & $\begin{array}{l}0061,7046 \\
7892,8425\end{array}$ \\
\hline $\begin{array}{l}24049 \\
24121\end{array}$ & 13 & $\begin{array}{l}878,20 \\
304,17\end{array}$ & $\begin{array}{l}24061 \\
24133\end{array}$ & $\begin{array}{r}10 \\
6\end{array}$ & 234 & $\begin{array}{l}244097 \\
24169\end{array}$ & 11 & $\begin{array}{l}3206,20662 \\
2710,2675\end{array}$ & $\begin{array}{l}24109 \\
24181\end{array}$ & 17 & $\begin{array}{r}2,23602 \\
17026,17621\end{array}$ \\
\hline 24 & & 20260 & 21327 & 5 & $\overline{2} 1$ & 2437 & 7 & 6071 & 24421 & 7 & $561-8242$ \\
\hline $\begin{array}{l}24469 \\
24697\end{array}$ & $\begin{array}{r}14 \\
5\end{array}$ & $\begin{array}{r}22360,15536 \\
15332,6724\end{array}$ & $\begin{array}{l}24481 \\
24709\end{array}$ & $1 \frac{1}{2}$ & $\begin{array}{r}15412,13511 \\
1,8516\end{array}$ & $\begin{array}{l}24517 \\
24733\end{array}$ & $\begin{array}{l}5 \\
2\end{array}$ & 21448,10313 & $\begin{array}{l}24649 \\
24781\end{array}$ & 2 & $\begin{array}{r}1106,6313 \\
1,11570\end{array}$ \\
\hline 24793 & 5 & 2044,16271 & 24841 & 14 & 20692,11495 & 24 & $\frac{2}{5}$ & $\begin{array}{r}1 \\
289\end{array}$ & & 11 & 21154,1313 \\
\hline $\begin{array}{l}25033 \\
25189\end{array}$ & $\begin{array}{r}5 \\
2\end{array}$ & $\begin{array}{r}11612,3961 \\
1,3131\end{array}$ & & $\begin{array}{l}5 \\
2\end{array}$ & $\begin{array}{l}56,2558 \\
2,23773\end{array}$ & $\begin{array}{l}25117 \\
25261\end{array}$ & 7 & $\begin{array}{l}2899,5405 \\
9092,12772\end{array}$ & $\begin{array}{l}25153 \\
25309\end{array}$ & 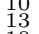 & $\begin{array}{l}24922,6530 \\
1369,19229\end{array}$ \\
\hline $\begin{array}{l}25321 \\
25561\end{array}$ & $\begin{array}{l}19 \\
11\end{array}$ & $\begin{array}{r}19334,17353 \\
9550,15542\end{array}$ & $\begin{array}{l}25357 \\
25609\end{array}$ & $\frac{2}{7}$ & $\begin{array}{r}1,2330 \\
6556,23363\end{array}$ & $\begin{array}{l}25453 \\
25621\end{array}$ & $\begin{array}{r}2 \\
10\end{array}$ & $\begin{array}{r}1,17276 \\
6478,19172\end{array}$ & $\begin{array}{l}25537 \\
25633\end{array}$ & $\begin{array}{r}10 \\
5\end{array}$ & $\begin{array}{r}18320,4567 \\
15532,21185\end{array}$ \\
\hline 25657 & 5 & & & 2 & 07 & 25 & 2 & & 25741 & 6 & 17596,3 \\
\hline $\begin{array}{l}25801 \\
25969\end{array}$ & 7 & 518 & & 11 & & & 10 & $\begin{array}{l}50 \\
72\end{array}$ & $\begin{array}{l}25933 \\
26029\end{array}$ & $\stackrel{2}{6}$ & 20000 \\
\hline $\begin{array}{l}26041 \\
26209\end{array}$ & 13 & 6356,13774 & $\begin{array}{l}26053 \\
26293\end{array}$ & $\begin{array}{l}2 \\
6\end{array}$ & $\begin{array}{r}1,13421 \\
1546,7430\end{array}$ & 26113 & 7 & $\begin{array}{r}404,9238 \\
22060\end{array}$ & 26161 & 13 & 20210,9586 \\
\hline 264 & 7 & 926,4547 & 26497 & $\begin{array}{l}0 \\
5\end{array}$ & 1, & & 2 & 1,1226 & & & 21484,26120 \\
\hline 26641 & 7 & 8024,23239 & 26701 & 22 & 15712,13418 & 26713 & 10 & 18358,9077 & 26737 & 10 & 22966,19802 \\
\hline $\begin{array}{l}26821 \\
26953\end{array}$ & $\frac{2}{7}$ & $\begin{array}{r}2,6376 \\
808,1400\end{array}$ & $\begin{array}{l}26833 \\
27061\end{array}$ & $\begin{array}{l}5 \\
2\end{array}$ & $\begin{array}{r}25156,4691 \\
1,4259\end{array}$ & $\begin{array}{l}26881 \\
27073\end{array}$ & $1 \frac{1}{5}$ & $\begin{array}{r}26152,17867 \\
8692,253446\end{array}$ & & 7 & $982,1,290$ \\
\hline 27241 & 17 & & 27253 & $\tilde{2}$ & 2,8710 & 272 & 6 & 2284,21 & 27337 & 5 & 127 \\
\hline $\begin{array}{l}27361 \\
27481\end{array}$ & $\begin{array}{l}7 \\
7\end{array}$ & $\begin{array}{r}21146 \\
17672\end{array}$ & & 7 & 8462,26809 & & $\begin{array}{l}13 \\
19\end{array}$ & $\begin{array}{l}22234, \\
7064,26838\end{array}$ & $\overline{2} 7$ & 11 & $\begin{array}{r}8776 \\
26026,\end{array}$ \\
\hline & 5 & & & 2 & 1,9989 & & 5 & 1602,140 & 27817 & 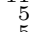 & 1,16328 \\
\hline & & 1,90 & & & & 27961 & 13 & 168,14954 & 27997 & 5 & 16556,13741 \\
\hline
\end{tabular}


Table 8: Table of $\gamma_{1}, \gamma_{2}$ for $\operatorname{RBIBD}(5 q+1,6,1)$ construction (cont.)

\begin{tabular}{|c|c|c|c|c|c|c|c|c|c|c|c|}
\hline & $\omega$ & $\gamma_{1}, \gamma_{2}$ & q & $\omega$ & $\gamma_{1}, \gamma_{2}$ & q & $\omega$ & $\gamma_{1}, \gamma_{2}$ & & $\omega$ & $\gamma_{1}, \gamma_{2}$ \\
\hline $\begin{array}{l}28057 \\
28297 \\
28477 \\
28561 \\
28657 \\
28789 \\
28921 \\
29101 \\
29221 \\
29437 \\
29629 \\
29881 \\
30013 \\
30169 \\
30313 \\
30529 \\
30649 \\
30781 \\
30853 \\
31069 \\
31189 \\
31333 \\
31489 \\
31729 \\
31957 \\
32077 \\
32257 \\
32401 \\
32569 \\
32761\end{array}$ & $\begin{array}{r}5 \\
5 \\
2 \\
* \\
5 \\
7 \\
11 \\
2 \\
2 \\
2 \\
7 \\
7 \\
2 \\
7 \\
5 \\
13 \\
7 \\
2 \\
2 \\
2 \\
13 \\
5 \\
7 \\
7 \\
2 \\
2 \\
15 \\
7 \\
7 \\
*\end{array}$ & $\begin{array}{r}1,19916 \\
1,4175 \\
2,24520 \\
11900,17422 \\
5584,27263 \\
23095,18800 \\
4856,1852 \\
2,16819 \\
1,22049 \\
1,11222 \\
23501,6397 \\
4586,24682 \\
1,18311 \\
17450,10954 \\
21214,12914 \\
5612,28000 \\
11572,6134 \\
1,7949 \\
1,13725 \\
1,15086 \\
3484,15732 \\
30956,14797 \\
8638,13253 \\
21548,16459 \\
1,4562 \\
2,3400 \\
15754,9191 \\
10090,6638 \\
19490,10921 \\
24934,30617\end{array}$ & $\begin{array}{l}28069 \\
28309 \\
28513 \\
28573 \\
28669 \\
28813 \\
28933 \\
29137 \\
29269 \\
29473 \\
29641 \\
29917 \\
30097 \\
30181 \\
30469 \\
30553 \\
30661 \\
30817 \\
30937 \\
31081 \\
31237 \\
31357 \\
31513 \\
31741 \\
31981 \\
32089 \\
32341 \\
32413 \\
32653\end{array}$ & $\begin{array}{r}7 \\
2 \\
5 \\
2 \\
6 \\
2 \\
2 \\
5 \\
6 \\
5 \\
7 \\
2 \\
10 \\
2 \\
2 \\
5 \\
2 \\
5 \\
15 \\
13 \\
6 \\
2 \\
7 \\
6 \\
6 \\
13 \\
2 \\
5 \\
2\end{array}$ & $\begin{array}{r}7490,10606 \\
1,22127 \\
22136,16180 \\
2,25849 \\
22144,28178 \\
2,3892 \\
1,9656 \\
2180,7261 \\
108770,2 \\
15812,22645 \\
1718,20311 \\
1,14357 \\
12164,29641 \\
5329,29351 \\
1,16979 \\
13934,7789 \\
1,20540 \\
14228,15202 \\
20056,1730 \\
22708,28232 \\
16054,1132 \\
1,5069 \\
31180,16577 \\
30268,16931 \\
8270,15340 \\
344,7162 \\
2,27484 \\
15232,10499 \\
1,26084\end{array}$ & $\begin{array}{l}28081 \\
28393 \\
28537 \\
28597 \\
28729 \\
28837 \\
29017 \\
29173 \\
29389 \\
29569 \\
29761 \\
29929 \\
30109 \\
30241 \\
30493 \\
30577 \\
30697 \\
30829 \\
30949 \\
31153 \\
31249 \\
31393 \\
31573 \\
31849 \\
32029 \\
32173 \\
32353 \\
32497 \\
32713\end{array}$ & $\begin{array}{r}19 \\
15 \\
5 \\
2 \\
22 \\
2 \\
5 \\
2 \\
2 \\
17 \\
17 \\
* \\
2 \\
11 \\
6 \\
5 \\
10 \\
2 \\
10 \\
10 \\
23 \\
5 \\
5 \\
14 \\
2 \\
5 \\
15 \\
7 \\
5\end{array}$ & $\begin{array}{r}2258,5971 \\
1540,20747 \\
27301,2339 \\
2,14152 \\
5704,17360 \\
1,25505 \\
1,11276 \\
2,19000 \\
1,22643 \\
27170,17158 \\
12880,18377 \\
1,22928 \\
1,28775 \\
10472,25018 \\
27863,29215 \\
1,2690 \\
18512,5626 \\
21,21455 \\
27802,9560 \\
17116,4904 \\
20,27442 \\
15572,13420 \\
29054,2242 \\
30238,26933 \\
13717,9266 \\
11794,18578 \\
31364,8821 \\
17752,14936 \\
18577,8342\end{array}$ & $\begin{array}{l}28201 \\
28429 \\
28549 \\
28621 \\
28753 \\
28909 \\
29077 \\
29209 \\
29401 \\
29581 \\
29833 \\
29989 \\
30133 \\
30253 \\
30517 \\
30637 \\
30757 \\
30841 \\
31033 \\
31177 \\
31321 \\
31477 \\
31657 \\
31873 \\
32041 \\
32233 \\
32377 \\
32533 \\
32749\end{array}$ & $\begin{aligned} 11 \\
2 \\
2 \\
13 \\
10 \\
2 \\
2 \\
7 \\
13 \\
10 \\
5 \\
2 \\
5 \\
2 \\
2 \\
2 \\
5 \\
7 \\
10 \\
7 \\
7 \\
6 \\
5 \\
11 \\
* \\
5 \\
5 \\
2 \\
2\end{aligned}$ & $\begin{array}{r}11596,27434 \\
1,3530 \\
1,26345 \\
13390,2225 \\
21868,10718 \\
26773,19544 \\
1,5912 \\
15704,17776 \\
25006,26942 \\
18955,4652 \\
14360,19414 \\
2,26377 \\
27092,23647 \\
1,10823 \\
1,15788 \\
1,14846 \\
25480,6938 \\
3944,12718 \\
1496,27679 \\
10100,26794 \\
2692,11633 \\
6286,18374 \\
1,18815 \\
5260,15536 \\
1,29984 \\
21944,4837 \\
14768,32086 \\
1,26279\end{array}$ \\
\hline
\end{tabular}

Table 9: Table of $m, w, c, c^{\prime}, c^{\prime \prime}$ for $\operatorname{BIBD}(q, 13,1)$ construction

\begin{tabular}{|c|c|c|c|c|c|c|c|c|c|c|c|}
\hline$q$ & $m$ & $w$ & $\mathrm{c}$ & $\mathrm{c}^{\prime}$ & $c^{\prime \prime}$ & $q$ & $m$ & $w$ & C & $\mathrm{C}^{\prime}$ & $C^{\prime \prime}$ \\
\hline $\begin{array}{r}6241 \\
9829 \\
15601 \\
16381 \\
18097 \\
20593 \\
21061 \\
21841 \\
22153 \\
22777 \\
23869 \\
24337 \\
25741 \\
26209 \\
27457 \\
28549 \\
29173 \\
30109 \\
31357 \\
31981\end{array}$ & $\begin{array}{r}1430 \\
6740 \\
4157 \\
12288 \\
2895 \\
1341 \\
20993 \\
9084 \\
8119 \\
14977 \\
13105 \\
19291 \\
7120 \\
16112 \\
4369 \\
18714 \\
10964 \\
26012 \\
4884 \\
5015\end{array}$ & $\begin{array}{r}23 \\
748 \\
3358 \\
3393 \\
12109 \\
143 \\
3375 \\
8529 \\
10588 \\
1740 \\
14848 \\
12508 \\
24210 \\
24180 \\
23868 \\
7759 \\
9972 \\
20130 \\
10608 \\
12636\end{array}$ & $\begin{array}{r}5276 \\
3942 \\
14688 \\
4508 \\
10205 \\
1651 \\
12210 \\
20542 \\
15483 \\
8251 \\
20525 \\
9143 \\
24046 \\
21052 \\
296 \\
6616 \\
19507 \\
21915 \\
10496 \\
12148\end{array}$ & $\begin{array}{r}5389 \\
3381 \\
10476 \\
6606 \\
1950 \\
10214 \\
152 \\
16054 \\
6187 \\
14704 \\
16395 \\
22389 \\
25039 \\
22252 \\
4005 \\
18676 \\
20561 \\
29240 \\
13281 \\
21725\end{array}$ & $\begin{array}{r}3429 \\
5212 \\
4395 \\
9926 \\
3646 \\
6166 \\
5448 \\
657 \\
16321 \\
20285 \\
21641 \\
23346 \\
24616 \\
7177 \\
3179 \\
1579 \\
9983 \\
2354 \\
17897 \\
25623\end{array}$ & $\begin{array}{r}8737 \\
14197 \\
16069 \\
16693 \\
19813 \\
20749 \\
21529 \\
21997 \\
22621 \\
23557 \\
24181 \\
25117 \\
26053 \\
26833 \\
28081 \\
29017 \\
29641 \\
30577 \\
31513\end{array}$ & $\begin{array}{r}1976 \\
6084 \\
4720 \\
3004 \\
2233 \\
6599 \\
14249 \\
3809 \\
14978 \\
9945 \\
5594 \\
21357 \\
22389 \\
6817 \\
4962 \\
7565 \\
8581 \\
5687 \\
13169\end{array}$ & $\begin{array}{r}2268 \\
5123 \\
14117 \\
5153 \\
7510 \\
3119 \\
3972 \\
11605 \\
13088 \\
22598 \\
155 \\
274 \\
12522 \\
18598 \\
1765 \\
1964 \\
19912 \\
28211 \\
10563\end{array}$ & $\begin{array}{r}2107 \\
12586 \\
7813 \\
9988 \\
9356 \\
3006 \\
2863 \\
16088 \\
20440 \\
21005 \\
12600 \\
16277 \\
24670 \\
18591 \\
9644 \\
18357 \\
17144 \\
13065 \\
4205\end{array}$ & $\begin{array}{r}4412 \\
6980 \\
1673 \\
14869 \\
1569 \\
4800 \\
19892 \\
20775 \\
17145 \\
2174 \\
13087 \\
19854 \\
3452 \\
7119 \\
21150 \\
4086 \\
29313 \\
20020 \\
25316\end{array}$ & $\begin{array}{r}8312 \\
6438 \\
2810 \\
13051 \\
13237 \\
9239 \\
16577 \\
20879 \\
22198 \\
19905 \\
11990 \\
9074 \\
9299 \\
21864 \\
21612 \\
19246 \\
18919 \\
15930 \\
16700\end{array}$ \\
\hline
\end{tabular}

Table 10: Table of $\gamma_{1}, \ldots, \gamma_{5}$ for $13-G D D 13^{q}$ construction

\begin{tabular}{|c|c|c|c|c|c|}
\hline$\underline{q}$ & $\omega$ & $71, \ldots, \gamma_{5}$ & 4 & $\omega$ & $\gamma_{1}, \ldots, \gamma_{5}$ \\
\hline & & $2,1,4,5,9$ & 121 & 6 & $4,73,98,119,15$ \\
\hline $\begin{array}{l}157 \\
277\end{array}$ & $\begin{array}{l}5 \\
5\end{array}$ & $\begin{array}{r}20,1,40,59,117 \\
218,247,40,149,207\end{array}$ & 229 & 6 & $\begin{array}{r}190,209,152,133,171 \\
305,4,16,331,57\end{array}$ \\
\hline 397 & 5 & $\begin{array}{r}218,247,40,149,207 \\
61,23,320,358,297\end{array}$ & $\begin{array}{l}361 \\
529\end{array}$ & * & $\begin{array}{r}305,4,116,331,57 \\
212,189,376,377,517\end{array}$ \\
\hline 601 & $\begin{array}{l}7 \\
2\end{array}$ & $223,155,52,291,2$ & 613 & 2 & $92,337,184,245,459$ \\
\hline $\begin{array}{l}661 \\
733\end{array}$ & $\frac{2}{6}$ & $\begin{array}{l}634,197,32,469,495 \\
602,667,484,53,549\end{array}$ & $\begin{array}{l}709 \\
757\end{array}$ & $2_{2}^{2}$ & $\begin{array}{l}236,649,118,59,177 \\
236,307,496,527,87\end{array}$ \\
\hline 829 & 2 & $496,152,261,557,583$ & 841 & * & $741,305,452,838,553$ \\
\hline & $\frac{2}{5}$ & $155,200,193,334,393$ & 877 & 2 & $765,734,167,718,607$ \\
\hline $\begin{array}{l}937 \\
997\end{array}$ & $\begin{array}{l}5 \\
7\end{array}$ & $\begin{array}{r}497,904,307,771,836 \\
586,55,9,923,944\end{array}$ & $\begin{array}{r}961 \\
1009\end{array}$ & 11 & $\begin{array}{l}787,376,543,767,704 \\
898,269,512,561,235\end{array}$ \\
\hline 1021 & 10 & $302,952,1,497,525$ & 1033 & $\begin{array}{r}1 \\
5 \\
5\end{array}$ & $803,393,446,904,565$ \\
\hline 1117 & $\frac{0}{2}$ & $885,299,340,1070,1093$ & $\begin{array}{l}1093 \\
1129\end{array}$ & 11 & $\begin{array}{r}527,358,800,783,1009 \\
856,992,573,437,709\end{array}$ \\
\hline $\begin{array}{l}1153 \\
1213\end{array}$ & $\begin{array}{l}5 \\
2 \\
2\end{array}$ & $\begin{array}{r}403,953,358,3,554 \\
911,760,1100,1179,931\end{array}$ & $\begin{array}{l}1201 \\
1237\end{array}$ & 11 & $\begin{array}{r}215,673,416,286,939 \\
1069,725,274,1166,669\end{array}$ \\
\hline $\begin{array}{l}1249 \\
1321\end{array}$ & 13 & $\begin{array}{r}520,375,1013,1040,1 \\
857,796,657,20,841\end{array}$ & $\begin{array}{l}1297 \\
1369\end{array}$ & 10 & $\begin{array}{r}971,91,8,262,783 \\
1306,853,734,443,1035\end{array}$ \\
\hline $\begin{array}{l}1381 \\
1453\end{array}$ & 2 & $\begin{array}{r}340,1196,751,53,585 \\
947,543,1366,973,464\end{array}$ & $\begin{array}{l}1429 \\
1489\end{array}$ & $\begin{array}{r}6 \\
14\end{array}$ & $164,227,1246,1185,163$ \\
\hline $\begin{array}{l}1453 \\
1549\end{array}$ & 2 & $821,361,1444,927,50$ & $\begin{array}{l}1489 \\
1597\end{array}$ & 11 & $28,1310,283,1565,1593$ \\
\hline $\begin{array}{l}1609 \\
1657\end{array}$ & 17 & $\begin{array}{r}1510,1093,1157,513,1358 \\
460,1370,509,1423,1533\end{array}$ & $\begin{array}{l}1621 \\
1669\end{array}$ & $\stackrel{2}{2}$ & $\begin{array}{r}1167,893,1582,691,2 \\
952,641,1447,609,1586\end{array}$ \\
\hline 1681 & * & $1244,502,561,1193,235$ & 1693 & 2 & $61,1409,1305,778,104$ \\
\hline $\begin{array}{l}1741 \\
1777\end{array}$ & $\frac{2}{5}$ & $719,1510,867,1154,577$ & $\begin{array}{l}1753 \\
1789\end{array}$ & 6 & $\begin{array}{r}52,781,893,1370,327 \\
1448,1199,814,469,651\end{array}$ \\
\hline 1801 & 11 & $1037,61,382,279,1142$ & 1849 & & $1625,525,52,1375,440$ \\
\hline $\begin{array}{l}1861 \\
1933\end{array}$ & 2 & $\begin{array}{l}1175,1531,1840,1454,3 \\
676,1339,1700,11,1329\end{array}$ & $\begin{array}{l}1873 \\
1993\end{array}$ & $\begin{array}{r}10 \\
5\end{array}$ & $\begin{array}{r}4,465,1667,1,1844 \\
1819,1858,1385,146,1203\end{array}$ \\
\hline $\begin{array}{l}2017 \\
2053\end{array}$ & 2 & $\begin{array}{r}875,481,297,964,44 \\
1660,819,623,1369,2\end{array}$ & $\begin{array}{l}2029 \\
2089\end{array}$ & $\stackrel{2}{7}$ & $\begin{array}{r}1959,527,1597,1498,2 \\
275,279,1303,190,1442\end{array}$ \\
\hline 2113 & 23 & $\begin{array}{r}725,1450,806,1485,391 \\
1651,837,701,760,326\end{array}$ & $\begin{array}{l}2137 \\
2197\end{array}$ & 10 & $634,1441,1329,395,1520$ \\
\hline 2209 & . & $1976,1540,179,1903,945$ & 2221 & 2 & $784,1484,1031,1881,529$ \\
\hline $\begin{array}{l}2269 \\
2293\end{array}$ & $2_{2}^{2}$ & $\begin{array}{l}1396,763,723,731,2 \\
640,759,899,1658,1\end{array}$ & $\begin{array}{l}2281 \\
2341\end{array}$ & 7 & $\begin{array}{r}1209,815,1186,979,38 \\
1966,1199,1465,2126,1215\end{array}$ \\
\hline 2377 & $\overline{5}$ & $1714,2084,421,719,1719$ & 2389 & 2 & $1948,1621,605,1209,2378$ \\
\hline 2473 & & $736,745,1942,53,656$ & 2521 & 17 & $\begin{array}{l}1180,1519,831,1709,2 \\
2275,998,389,2284,855\end{array}$ \\
\hline $\begin{array}{l}2557 \\
2617\end{array}$ & 5 & $\begin{array}{r}1525,2045,2163,232,2 \\
1883,255,157,532,1844\end{array}$ & $\begin{array}{l}2593 \\
2677\end{array}$ & $\begin{array}{l}7 \\
2 \\
\end{array}$ & $657,2494,2587,1391,2048$ \\
\hline & 19 & $1699,610,305,951,1244$ & 2713 & 5 & $1135,1990,1535,471,1916$ \\
\hline 309 & ${ }_{*}^{6}$ & $\begin{array}{l}399,1414,1075,495 \\
1157,8,249,2806,1\end{array}$ & 2797 & 2 & $\begin{array}{l}9,1125,1190,1570,1 \\
65,2721,1570,206,1\end{array}$ \\
\hline 2857 & 11 & $345,647, ' 883,1234,1598$ & 2917 & 5 & $1300,955,881,2282,825$ \\
\hline
\end{tabular}


Table 10: Table of $\gamma_{1}, \ldots, \gamma_{5}$ for 13 -GDD $13^{q}$ construction (cont.)

\begin{tabular}{|c|c|c|c|c|c|}
\hline 2953 & 13 & $\begin{array}{r}\gamma_{1}, \ldots, \gamma 5 \\
73,1382,2920,431,2511\end{array}$ & 3001 & 14 & $\begin{array}{r}\gamma 1, \ldots, \gamma 5 \\
, 1712,2765,2995,2967\end{array}$ \\
\hline 3037 & 2 & $41,2626,411,1472,1$ & 3049 & 11 & $1709,1561,2344,2133,2048$ \\
\hline $\begin{array}{l}3061 \\
3121\end{array}$ & $\overline{6}$ & $\begin{array}{l}1870,2744,113,2299,1815 \\
505,2067,1804,1139,1142\end{array}$ & 3109 & 6 & $\begin{array}{r}2575,1072,2777,1167,1694 \\
425,2439,1555,1624,548\end{array}$ \\
\hline $\begin{array}{l}3121 \\
3181 \\
3200\end{array}$ & 7 & $505,2063,928,517,71,3062$ & $\begin{array}{l}1169 \\
3217\end{array}$ & 5 & $425,2439,1555,1624,248$ \\
\hline $\begin{array}{l}3229 \\
3301\end{array}$ & $\begin{array}{l}6 \\
6\end{array}$ & $\begin{array}{r}793,1642,2258,1955,93 \\
3027,142,2147,1495,866\end{array}$ & $\begin{array}{l}3253 \\
3313\end{array}$ & $\begin{array}{r}2 \\
10\end{array}$ & $\begin{array}{r}1999,225,2260,2531,2 \\
3153,2113,1582,2525,1688\end{array}$ \\
\hline $\begin{array}{l}3361 \\
3433\end{array}$ & $\begin{array}{r}22 \\
5\end{array}$ & $\begin{array}{l}1888,2565,1651,503,1100 \\
765,1516,1265,1333,2126\end{array}$ & $\begin{array}{l}3373 \\
3457\end{array}$ & $\begin{array}{r}5 \\
7\end{array}$ & $\begin{array}{r}1684,2331,689,914,1 \\
2902,95,2839,2660,813\end{array}$ \\
\hline 3469 & 2 & $3371,116,724,939,1$ & 3481 & * & $2159,260,976,759,1$ \\
\hline $\begin{array}{l}3517 \\
3541\end{array}$ & $\stackrel{2}{7}$ & $\begin{array}{r}3233,3091,1737,1906,2 \\
3367,430,1190,1223,1035\end{array}$ & $\begin{array}{l}3529 \\
3613\end{array}$ & $\begin{array}{r}17 \\
2\end{array}$ & $41,2353,1191,2146,1682$ \\
\hline $\begin{array}{l}3647 \\
3697 \\
3697\end{array}$ & $\begin{array}{l}2 \\
5\end{array}$ & $\begin{array}{l}3371,3388,1634,579,1 \\
3262,3439,417,1853,1838\end{array}$ & $\begin{array}{l}3010 \\
3673 \\
3709\end{array}$ & 5 & $1101,1409,820,619,2024$ \\
\hline 3721 & $*$ & $2133,1451,289,1918,1364$ & 3733 & 2 & $\begin{array}{r}3446,1180,593,1923,1 \\
121,2981,2122,1923,2\end{array}$ \\
\hline $\begin{array}{l}3769 \\
3853\end{array}$ & $\begin{array}{l}7 \\
2\end{array}$ & $\begin{array}{r}2303,2901,2455,3238,878 \\
1545,16,3559,401,2\end{array}$ & $\begin{array}{l}3793 \\
3877\end{array}$ & 5 & $\begin{array}{r}2045,897,409,160,1238 \\
3755,2404,3098,423,1\end{array}$ \\
\hline 3889 & $1 \frac{1}{5}$ & $3653,2392,1735,1311,1046$ & 4021 & 2 & $1318,819,2096,3605,1$ \\
\hline $\begin{array}{l}4059 \\
4129\end{array}$ & 13 & $\begin{array}{r}419,3224,1744,717,1945 \\
2236,665,3578,601,2223\end{array}$ & 4153 & 5 & $2615,1557,520,3866,1$ \\
\hline $\begin{array}{l}4177 \\
4261\end{array}$ & $\begin{array}{l}5 \\
2\end{array}$ & $\begin{array}{r}3525,2288,2183,2545 \\
2672,4234,1385,669,1\end{array}$ & 4201 & $1 \frac{1}{5}$ & $\begin{array}{l}3723,4168,907,227,776 \\
538,3625,2157,2705,680\end{array}$ \\
\hline 4297 & 25 & $\begin{array}{l}1945,777,796,773,992 \\
2311,2061,2740,3356\end{array}$ & 4357 & ${ }_{*}^{2}$ & $512,1563,2909,1438,1$ \\
\hline 4513 & 7 & $1438,3674,217,1601,2019$ & 4549 & 6 & $\begin{array}{l}901,4126,841,4065,476 \\
306,2041,520,3083,267\end{array}$ \\
\hline $\begin{array}{l}4561 \\
4621\end{array}$ & ${ }_{2}^{11}$ & $\begin{array}{l}217,3994,819,173,608 \\
2943,1234,1286,341,1\end{array}$ & $\begin{array}{l}4597 \\
4657\end{array}$ & $\begin{array}{r}5 \\
15\end{array}$ & $\begin{array}{l}3,4147,3932,1876,1245 \\
24,3884,911,2479,2715\end{array}$ \\
\hline 4729 & 17 & $\begin{array}{r}4438,4141,4295,2288,4359 \\
4012,4489,335,3681,4796\end{array}$ & 4789 & 2 & $035,2026,1862,4349,1$ \\
\hline 4861 & $1 \frac{1}{2}$ & $\begin{array}{l}1864,4124,1907,2383,657 \\
3883,1522,3401,1101,2\end{array}$ & 4909 & 6 & $3331,802,335,4802,3657$ \\
\hline $\begin{array}{l}4933 \\
4969\end{array}$ & 11 & $2686,4131,4429,131,4172$ & 4957 & $\frac{2}{5}$ & $\begin{array}{r}981,1330,37,3563,2 \\
2823,2770,3953,1736,1\end{array}$ \\
\hline $\begin{array}{l}5041 \\
5101\end{array}$ & 6 & $4822,3795,1181,4334,139$ & 5077 & $\stackrel{2}{19}$ & $4583,2935,27,2650,2$ \\
\hline 5197 & 7 & $4589,2065,2800,3752,5175$ & 5209 & 17 & $5165,646,5089,1053,2102$ \\
\hline $\begin{array}{l}5233 \\
5329\end{array}$ & 10 & $\begin{array}{r}3033,2777,4228,4945,2552 \\
971,3166,1621,4587,1184\end{array}$ & $\begin{array}{l}5281 \\
5413\end{array}$ & 7 & $\begin{array}{r}891,2875,4702,4889,4898 \\
1346,1198,1577,4471,4485\end{array}$ \\
\hline $\begin{array}{l}5329 \\
5437 \\
5521\end{array}$ & 10 & $148,3341,1129,386,327$ & 5449 & 7 & $\begin{array}{r}1346,1198,15777,4471,4485 \\
\quad 879,187,3676,5387,4034\end{array}$ \\
\hline $\begin{array}{l}5521 \\
5569\end{array}$ & $\begin{array}{l}11 \\
13\end{array}$ & $\begin{array}{r}523,4919,4174,1215,3692 \\
2648,59,3562,3675,1\end{array}$ & 5557 & $\begin{array}{l}2 \\
6\end{array}$ & $5327,1912,1550,481,4995$ \\
\hline $\begin{array}{l}5641 \\
5689\end{array}$ & $\begin{array}{l}14 \\
11\end{array}$ & $\begin{array}{r}391,4865,1708,5163,2180 \\
724,3799,5151,5633,482\end{array}$ & $\begin{array}{l}5653 \\
5701\end{array}$ & $\begin{array}{l}5 \\
2\end{array}$ & $\begin{array}{r}350,4090,1535,2479,3117 \\
4726,3740,4553,183,1\end{array}$ \\
\hline 5737 & $\begin{array}{r}5 \\
6\end{array}$ & $\begin{array}{r}4876,3344,627,5489,1777 \\
620,1181,718,2071,2685\end{array}$ & 5749 & 2 & $\begin{array}{r}1318,5660,2753,3363,1 \\
2485,4163,2806,1923,4034\end{array}$ \\
\hline 5869 & 2 & $2995,320,923,789,1228,1$ & 5881 & 31 & $1213,808,5013,4667,5534$ \\
\hline 6073 & 10 & $3363,4559,916,1861,5816$ & 6121 & $\begin{array}{r}5 \\
7 \\
\end{array}$ & $\begin{array}{r}2428,5357,188,277,1485 \\
5083,4234,5627,171,4790\end{array}$ \\
\hline $\begin{array}{l}6133 \\
6229\end{array}$ & $\begin{array}{l}5 \\
2\end{array}$ & $\begin{array}{r}1271,3889,4778,1558,891 \\
921,3562,3188,1205,1\end{array}$ & $\begin{array}{l}6217 \\
6241\end{array}$ & 5 & $\begin{array}{r}2948,4204,5277,4079,1 \\
2890,735,3167,1021,320\end{array}$ \\
\hline 6277 & 2 & $548,4223,4017,556,1$ & 6301 & 10 & $1607,3464,4195,2824,1737$ \\
\hline 6373 & 2 & $944,4,2643,2777,1$ & $\begin{array}{l}0301 \\
6397 \\
6160\end{array}$ & $\begin{array}{r}19 \\
2\end{array}$ & $42,3291,5,6081,1936,1$ \\
\hline 6481 & 7 & $\begin{array}{l}4600,1166,4223,691,5661 \\
759,3172,3881,2035,1406\end{array}$ & $\begin{array}{l}6469 \\
6529 \\
629\end{array}$ & 7 & \\
\hline $\begin{array}{l}6553 \\
6637\end{array}$ & $\begin{array}{r}10 \\
2\end{array}$ & $\begin{array}{r}4705,2453,5547,1828,392 \\
1961,6580,4933,5709,2\end{array}$ & $\begin{array}{l}6577 \\
6661\end{array}$ & $\begin{array}{l}5 \\
6\end{array}$ & $\begin{array}{r}6317,5991,2995,2950,974 \\
5944,3668,2323,3521,1497\end{array}$ \\
\hline $\begin{array}{l}6673 \\
6733\end{array}$ & $\begin{array}{r}5 \\
2\end{array}$ & $\begin{array}{r}2757,508,1217,536,1 \\
878,3573,3077,6010,1\end{array}$ & $\begin{array}{l}6709 \\
6781\end{array}$ & 2 & $6551,3832,277,2403,2$ \\
\hline 6793 & 10 & $2566,1,3389,2613,4856$ & $\begin{array}{l}6829 \\
6829\end{array}$ & 2 & $2517,650,4949,5848,1$ \\
\hline 6841 & 22 & $3197,139,5128,4803,5756$ & $\begin{array}{l}6889 \\
6961\end{array}$ & 13 & $2476,2459,5625,1268,1$ \\
\hline 6997 & $\begin{array}{l}2 \\
5 \\
2\end{array}$ & $5111,1324,986,1111,855$ & $\begin{array}{l}6901 \\
7057 \\
7100\end{array}$ & $\begin{array}{r}13 \\
5 \\
7\end{array}$ & $2266,2351,5234,3627,1$ \\
\hline $\begin{array}{l}7069 \\
7177\end{array}$ & 10 & $\begin{array}{r}1216,4238,6743,6327,6493 \\
4397,610,5163,6841, \\
1964\end{array}$ & $\begin{array}{l}7129 \\
7213\end{array}$ & $\begin{array}{l}7 \\
5\end{array}$ & $\begin{array}{r}5267,2176,3903,1729,878 \\
5528,6597,5531,7006,1\end{array}$ \\
\hline $\begin{array}{l}7237 \\
7309\end{array}$ & $\begin{array}{l}2 \\
6\end{array}$ & $\begin{array}{r}1070,4276,1463,5817,1 \\
443,2506,1295,253,2402\end{array}$ & $\begin{array}{l}7297 \\
7321\end{array}$ & $\begin{array}{l}5 \\
7\end{array}$ & $\begin{array}{r}1675,4833,893,5980,2054 \\
99,5555,157,3574,3158\end{array}$ \\
\hline 7333 & 6 & $\begin{array}{r}6277,4138,4376,521,4137 \\
4197,2141,150,7111\end{array}$ & 7369 & 7 & $6353,2767,5865,142,1316$ \\
\hline $\begin{array}{l}7393 \\
7477 \\
\end{array}$ & $\begin{array}{l}5 \\
2\end{array}$ & $\begin{array}{r}4197,2141,1150,7111,5768 \\
2950,2906,3345,4985,1\end{array}$ & $\begin{array}{l}7417 \\
7489\end{array}$ & $\begin{array}{l}5 \\
7\end{array}$ & $\begin{array}{l}5439,1678,7039,209,1490 \\
2217,568,5351,5449,1520\end{array}$ \\
\hline $\begin{array}{l}7537 \\
7561\end{array}$ & $\begin{array}{r}7 \\
13\end{array}$ & $\begin{array}{r}1574,697,2843,1768,6267 \\
4133,5050,3423,4837,458\end{array}$ & $\begin{array}{l}7549 \\
7573\end{array}$ & 2 & $\begin{array}{r}1155,1786,1208,1097,1 \\
1163,3585,1687\end{array}$ \\
\hline 7621 & 2 & $1949,5398,7496,4797,1$ & 7669 & 2 & $201,4595,1468,5497,2$ \\
\hline 7681 & 17 & $\begin{array}{l}3145,2602,483,35,7448 \\
640,56,2335,1931,3243\end{array}$ & 7717 & 12 & $\begin{array}{r}952,2283,2371,2249,2 \\
1498,669\end{array}$ \\
\hline 7789 & 2 & $7508,4725,5147,5314,1$ & 7873 & 5 & $4190,1522,3947,7137,1$ \\
\hline $\begin{array}{l}7921 \\
7993\end{array}$ & 5 & $\begin{array}{r}2818,5487,2900,6245,1 \\
1037,4609,4413,3514,6080\end{array}$ & $\begin{array}{l}7933 \\
8017\end{array}$ & $\frac{2}{5}$ & $\begin{array}{r}7157,4688,1527,1594,1 \\
3929,6883,6268,5259,7496\end{array}$ \\
\hline 8053 & 2 & $\begin{array}{r}4083,3923,3094,4699,2 \\
5864,3970,2113,989,4131\end{array}$ & 8089 & 17 & $\begin{array}{r}6832,641,6805,2451,4958 \\
6940,1238,7331,6435,1\end{array}$ \\
\hline 8209 & 7 & $7601,1,411,7948,4124$ & $\begin{array}{l}8101 \\
8221\end{array}$ & 2 & $844,1365,4883,4567,2$ \\
\hline 8233 & $\begin{array}{r}10 \\
2\end{array}$ & $\begin{array}{r}2086,7133,6763,3567,6032 \\
8051,3,6770,1426,1\end{array}$ & $\begin{array}{l}8269 \\
8317\end{array}$ & $\begin{array}{l}2 \\
6\end{array}$ & $\begin{array}{l}1013,3830,2098,7623,1 \\
5308,1,4376,3227,7911\end{array}$ \\
\hline 8329 & 7 & $7973,1522,273,6289,2894$ & 8353 & 5 & $4653,4078,5024,4307,1$ \\
\hline 8461 & 6 & $7957,7955,2049,4990,1490$ & 8521 & $\begin{array}{r}6 \\
13\end{array}$ & $\begin{array}{r}4739,1,5434,2612,5235 \\
5831,5323,3201,3166,5282\end{array}$ \\
\hline 85811 & 17 & $\begin{array}{r}3208,1,7751,7569,7358 \\
2323,6071,7204,6219,2588\end{array}$ & $\begin{array}{l}8629 \\
8677\end{array}$ & $\begin{array}{l}6 \\
2\end{array}$ & $\begin{array}{r}1493,3248,1510,2329,3483 \\
4865,598,5421,4273,2\end{array}$ \\
\hline 8689 & 13 & $2547,1129,394,7991,7484$ & 8713 & 5 & $7883,5132,6970,2163,1$ \\
\hline 8821 & ${ }_{2}^{5}$ & $\begin{array}{l}8620,1760,4097,3693,1 \\
2853,2558,1552,7193,1\end{array}$ & $\begin{array}{l}8761 \\
8893\end{array}$ & $\begin{array}{r}23 \\
5\end{array}$ & $\begin{array}{r}4215,3484,3919,8735,7568 \\
3374,5637,2284,2627,1\end{array}$ \\
\hline $\begin{array}{l}8929 \\
9001\end{array}$ & $1 \frac{11}{7}$ & $\begin{array}{l}1137,4030,5549,7136,1 \\
4805,6578,4696,2397,\end{array}$ & $\begin{array}{l}8941 \\
9013\end{array}$ & 6 & $\begin{array}{l}3616,1733,5888,1633,8007 \\
1997,5744,6931,7024,2007\end{array}$ \\
\hline 9049 & 7 & $2632,8579,2875,6219,476$ & 9109 & 10 & $2881,1294,8126,7865,6165$ \\
\hline $\begin{array}{l}9133 \\
9181 \\
-130\end{array}$ & $\begin{array}{l}6 \\
2\end{array}$ & $3463,7312,3665,4268,1821$ & $\begin{array}{l}9157 \\
9241\end{array}$ & 13 & $\begin{array}{l}7774,3039,2551,1367,3038 \\
3199,3448,6035,3801,1724\end{array}$ \\
\hline $\begin{array}{l}9277 \\
9349\end{array}$ & $\begin{array}{l}5 \\
2\end{array}$ & $\begin{array}{r}2927,5668,5318,213,1 \\
3465,2,4108,6617,1\end{array}$ & 9337 & $\begin{array}{l}5 \\
2\end{array}$ & $4231,8061,2638,1379,3788$ \\
\hline 409 & . & $5535,3701,2044,2275,3332$ & 9421 & 2 & $2479,2542,5015,783,2$ \\
\hline 9433 & 5 & $4185,1930,2329,1865,9386$ & 9601 & 13 & $4179,8104,8735,7339,4052$ \\
\hline $\begin{array}{l}9613 \\
9661\end{array}$ & 2 & $\begin{array}{r}262,765,6659,9601,2 \\
8187,3110,8069,9430,1\end{array}$ & 9697 & 10 & $\begin{array}{l}4675,7546,909,5915,5240 \\
3139,6771,8663,3316,5816\end{array}$ \\
\hline $\begin{array}{l}9721 \\
9769\end{array}$ & $\begin{array}{r}7 \\
13\end{array}$ & $\begin{array}{r}3465,563,6880,6110,1 \\
2777,5194,3571,9483,3200\end{array}$ & $\begin{array}{l}9733 \\
9781\end{array}$ & $\begin{array}{l}2 \\
6\end{array}$ & $\begin{array}{l}6674,5,8038,2763,1 \\
1718,5926,4673,2059,8475\end{array}$ \\
\hline 9817 & $\begin{array}{r}5 \\
2\end{array}$ & $\begin{array}{r}9469,2,9148,4577,4443 \\
1834,9133,1059,9683\end{array}$ & $\begin{array}{l}9829 \\
9949\end{array}$ & 10 & $4370,4546,6687,5969,8407$ \\
\hline 9973 & 11 & $6520,4903,9071,3662,3093$ & 10009 & 11 & $6817,6207,8824,2273,7316$ \\
\hline $\begin{array}{l}10069 \\
10141\end{array}$ & $2_{2}^{2}$ & $\begin{array}{l}2056,2067,6362,6713,1 \\
3969,5740,1232,7697,1\end{array}$ & $\begin{array}{l}10093 \\
10177\end{array}$ & $\frac{2}{7}$ & $\begin{array}{r}6190,6584,7863,2381,1 \\
9400,7124,4405,2315,3993\end{array}$ \\
\hline 10201 & * & $9405,3899,1160,9808,1$ & 10273 & 10 & $8907,346,2515,5711,7220$ \\
\hline $\begin{array}{l}10321 \\
10357\end{array}$ & 2 & $1279,4923,2231,7870,2$ & & 13 & $5961,2716,995,6163,6818$ \\
\hline 1042 & & $2225,3010,6566,1411,7095$ & 10453 & & $0052,9964,7733,303,325$ \\
\hline
\end{tabular}


Table 10: Table of $\gamma_{1}, \ldots, \gamma_{5}$ for 13 -GDD $13^{q}$ construction (cont.)

\begin{tabular}{|c|c|c|c|c|c|}
\hline 10 & 2 & $\begin{array}{r}\gamma_{1}, \ldots, \gamma_{5} \\
2347,2703,6329,2\end{array}$ & 10501 & 2 & $\begin{array}{r}\gamma 1, \ldots, \gamma 5 \\
5092,7214,1889,3045,1\end{array}$ \\
\hline 10513 & 7 & $667,10300,8949,17,6284$ & 10007 & & $\begin{array}{l}5092,7214,1889, \\
7725,2794,5741,4232,1\end{array}$ \\
\hline 10609 & & $7241,208,3735,9541,9152$ & 10657 & 7 & $3892,1,716,9857,9501$ \\
\hline $\begin{array}{l}10729 \\
10789\end{array}$ & $\begin{array}{l}7 \\
2\end{array}$ & $\begin{array}{c}2597,5218,1735,8523,10004 \\
3621,9370,3764,5813,1\end{array}$ & $\begin{array}{l}10753 \\
10837\end{array}$ & $1 \frac{1}{2}$ & $\begin{array}{r}4370,7025,8671,9082,5499 \\
10499,10256,8416,9819,1\end{array}$ \\
\hline $\begin{array}{l}10861 \\
10957\end{array}$ & $\overline{5}$ & $\begin{array}{l}7874,3802,6413,2685,1 \\
5657,4732,8073,4682,1\end{array}$ & $\begin{array}{l}10909 \\
10993\end{array}$ & $\begin{array}{l}\overline{2} \\
7\end{array}$ & $5767, \begin{array}{l}6975,6086,953,4708,1 \\
10335,9046,5633,8420\end{array}$ \\
\hline 11113 & 13 & $1673,10982,6982,3793,393$ & 11149 & 10 & $9781,3316,10973,8450,6813$ \\
\hline 11197 & $\frac{1}{2}$ & $3469,10721,7149,5374,2$ & $\begin{array}{l}1113 \\
11257\end{array}$ & 10 & $9147,2776,4067,7147,3272$ \\
\hline $\begin{array}{l}11317 \\
11353\end{array}$ & $\frac{2}{7}$ & $8629,10726,10593,7595,2834$ & $\begin{array}{l}11329 \\
11437\end{array}$ & $\begin{array}{l}7 \\
2 \\
2\end{array}$ & $\begin{array}{r}213,6988,7721,10508,1 \\
320,2109,3965,6706,1\end{array}$ \\
\hline $\begin{array}{l}11449 \\
11593\end{array}$ & $\begin{array}{l}* \\
5\end{array}$ & $\begin{array}{r}6412,5726,6639,3527,1 \\
11291,11320\end{array}$ & $\begin{array}{l}11497 \\
11617\end{array}$ & $\begin{array}{r}7 \\
10\end{array}$ & $\begin{array}{r}7287,11123,8938,49,830 \\
5275,5416,10059,9725,6992\end{array}$ \\
\hline 11701 & 6 & $\begin{array}{r}7840,4501,3135,10331,2 \\
5817,4330,9055,6545,9536\end{array}$ & $\begin{array}{l}11689 \\
11821\end{array}$ & $\begin{array}{l}7 \\
2\end{array}$ & $\begin{array}{r}4859,6154,6565,1671,9446 \\
4139,3,1226,8260,2023\end{array}$ \\
\hline $\begin{array}{l}11833 \\
11941\end{array}$ & $\begin{array}{r}5 \\
10\end{array}$ & $\begin{array}{l}078,10259,11361,9961,8684 \\
782,2273,10995,11906,5575\end{array}$ & $\begin{array}{l}11881 \\
11953\end{array}$ & 5 & $10097,1210,4101,9145,8360$ \\
\hline 12037 & 5 & $10313,5084,5901,4726,3043$ & 12049 & 13 & $10717,10048,2733,2099,9290$ \\
\hline 12073 & 6 & $\begin{array}{r}7642,8768,3403,875,3219 \\
11224,1,6845,10160,10953\end{array}$ & $\begin{array}{l}12097 \\
12157\end{array}$ & $\begin{array}{r}5 \\
2\end{array}$ & $\begin{array}{r}4317,50,3581,3406,1 \\
10697,11924,6730,2199,1\end{array}$ \\
\hline $\begin{array}{l}12241 \\
12277 \\
12301\end{array}$ & $\begin{array}{l}7 \\
2 \\
2 \\
\end{array}$ & $\begin{array}{r}8695,4774,131,2259,1994 \\
2477,7462,2420,807,1\end{array}$ & $\begin{array}{l}12253 \\
12289 \\
12373\end{array}$ & $1 \underset{2}{2}$ & $\begin{array}{r}10239,4888,545,4952,1 \\
4579,3472,32311529,4172 \\
8433962\end{array}$ \\
\hline 12409 & 7 & $10269,2488,383,7537,7448$ & 12421 & 7 & $9223,3604,7697,1388,10581$ \\
\hline 2433 & $\begin{array}{r}13 \\
6\end{array}$ & $\begin{array}{r}10913,1,8362,2697,6362 \\
11585,2404,4442,10651,10113\end{array}$ & $\begin{array}{l}12457 \\
12541\end{array}$ & $\begin{array}{l}10 \\
14\end{array}$ & $\begin{array}{r}8753,3400,6709,9,6800 \\
4712,6400,11253,863,4735\end{array}$ \\
\hline 12553 & $\begin{array}{l}5 \\
2 \\
2\end{array}$ & $\begin{array}{r}4555,7852,8763,8177,9350 \\
9069,38,11554,12437,1\end{array}$ & $\begin{array}{l}12577 \\
12601\end{array}$ & $\begin{array}{l}10 \\
11\end{array}$ & $9855,8836,11513,4081,10718$ \\
\hline $\begin{array}{l}12613 \\
12697\end{array}$ & $\frac{2}{7}$ & $4513,8254,12689,8445,2468$ & $\begin{array}{l}12637 \\
12721\end{array}$ & $\begin{array}{r}2 \\
13\end{array}$ & $\begin{array}{r}11750,9256,5535,1871,1 \\
11773,6280,7433,9129,1934\end{array}$ \\
\hline $\begin{array}{l}12757 \\
12781\end{array}$ & 2 & $1991,4261,10096,8457,2$ & $\begin{array}{l}12769 \\
12829\end{array}$ & 2 & $\begin{array}{r}5753,2631,1798,4526,1 \\
6239,6944,8733,922,1\end{array}$ \\
\hline 12841 & $\begin{array}{l}21 \\
13\end{array}$ & $\begin{array}{r}10096,1,11957,7737,9590 \\
11357,9982,7963,10251,5864\end{array}$ & $\begin{array}{l}12853 \\
12973\end{array}$ & $\begin{array}{r}5 \\
14\end{array}$ & $\begin{array}{r}9506,11871,9844,6965,1 \\
10349,10996,3031,12866,237\end{array}$ \\
\hline 13009 & $\begin{array}{l}7 \\
6\end{array}$ & $\begin{array}{r}9322,12525,617,9343,2582 \\
7240,1,3209,11480,5931\end{array}$ & $\begin{array}{l}13033 \\
13177\end{array}$ & 5 & $5, \begin{array}{r}10012,1927,10983,5006 \\
8962,8720,603,6737,1\end{array}$ \\
\hline 13249 & 7 & $4685,11025,7900,7243,11582$ & 13297 & 5 & $2360,11794,6155,4023,12055$ \\
\hline $\begin{array}{l}13309 \\
13417\end{array}$ & 6 & $\begin{array}{r}10667,640,10381,5288,639 \\
3769,12574,3533,13101,10640\end{array}$ & $\begin{array}{l}13381 \\
13441\end{array}$ & $\begin{array}{l}10 \\
11\end{array}$ & $\begin{array}{r}658,2330,7595,3849,7855 \\
6493,8032,8651,13383,13328\end{array}$ \\
\hline $\begin{array}{l}13477 \\
13537\end{array}$ & 7 & $\begin{array}{r}6733,2578,5673,8891,2 \\
6519,5530,7385,13435,9542\end{array}$ & 13513 & $\begin{array}{r}1 \\
5\end{array}$ & $863,1240,2612,13161,1$ \\
\hline 13633 & 5 & $3893,4660,10101,12098,1$ & $\begin{array}{l}1359 \\
13669\end{array}$ & 6 & $862,2756,7693,8909,723$ \\
\hline $\begin{array}{l}13681 \\
13729\end{array}$ & 22 & $\begin{array}{r}12161,12352,3097,8378,2769 \\
11331,11812,3691,125,4220\end{array}$ & $\begin{array}{l}13693 \\
13789\end{array}$ & 6 & $\begin{array}{r}215,6652,829,1238,7041 \\
6820,489,10753,12431,10700\end{array}$ \\
\hline $\begin{array}{l}13873 \\
13933\end{array}$ & $\begin{array}{r}5 \\
2\end{array}$ & 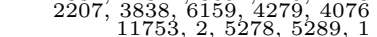 & 13921 & 7 & \\
\hline 14149 & 6 & $4133,13268,7540,3019,2205$ & 14173 & 20 & $11009,6339,2068,2587,2$ \\
\hline 14281 & 19 & $381,7468,9777,725,12674$ & 14293 & 6 & $9688,2936,3287,13261,12825$ \\
\hline $\begin{array}{l}14341 \\
14401\end{array}$ & $\stackrel{2}{1}$ & $\begin{array}{r}12058,2411,5929,9285,2 \\
99,10264,12207,4373,2966\end{array}$ & $\begin{array}{l}14389 \\
14437\end{array}$ & 2 & $\begin{array}{r}5565,3196,11522,2987,1 \\
198,8989,13529,3268,1689\end{array}$ \\
\hline $\begin{array}{l}14449 \\
14533\end{array}$ & $\begin{array}{r}22 \\
2\end{array}$ & $3793,7912,11661,2945,3956$ & $\begin{array}{l}14461 \\
14557\end{array}$ & $\begin{array}{l}2 \\
2\end{array}$ & $\begin{array}{r}12538,12488,3605,5193,1 \\
8693,8662,949,2541,2\end{array}$ \\
\hline 14593 & $\overline{5}$ & $8919,4184,832,3431,1$ & 14629 & $\overline{2}$ & $12635,9776,11890,13401,13699$ \\
\hline $\begin{array}{l}14641 \\
14713\end{array}$ & 5 & $\begin{array}{r}13628,8781,12760,2183,1 \\
14411,13996,759,2923,13280\end{array}$ & $\begin{array}{l}14653 \\
14737\end{array}$ & $\begin{array}{r}2 \\
10\end{array}$ & $\begin{array}{r}7517,3092,5182,10527,1 \\
14483,1588,9177,1291,3176\end{array}$ \\
\hline $\begin{array}{l}14797 \\
14869\end{array}$ & $\begin{array}{l}2 \\
2\end{array}$ & $\begin{array}{r}3988,11867,12038,12813,1 \\
983,1838,4479,10984,1\end{array}$ & $\begin{array}{l}14821 \\
14929\end{array}$ & $\begin{array}{r}2 \\
7\end{array}$ & $\begin{array}{r}9781,7677,9778,8819,2 \\
3860,12568,2105,6633,1\end{array}$ \\
\hline 15013 & 2 & $\begin{array}{r}6383,4,11762,13167,1 \\
1031\end{array}$ & 31 & 2 & $5417,12561,14528,11380,1$ \\
\hline 15193 & 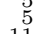 & $3275,3362,5055,13234,1$ & $\begin{array}{l}15121 \\
15217\end{array}$ & 10 & $14725,12508,15083,13305,3668$ \\
\hline $\begin{array}{l}15241 \\
15289\end{array}$ & ${ }_{11}^{11}$ & $\begin{array}{r}13799,7000,8307,3265,11120 \\
8769,2422,5189,12217,338\end{array}$ & $\begin{array}{l}15277 \\
15313\end{array}$ & 6 & $\begin{array}{r}2569,14104,8285,12674,1173 \\
7054,11456,12633,1769,1\end{array}$ \\
\hline 15349 & $\frac{2}{2}$ & $7941,12485,1054,1142,1$ & 15361 & 7 & $1158,9029,9025,11631,2696$ \\
\hline 15541 & 6 & $12461,8080,9865,13107,11810$ & 15601 & 23 & $1142,5374,4081,1109,5055$ \\
\hline 15625 & * & $3820,11714,6323,7011,1$ & 15649 & 11 & $8515,14746,14999,1335,2720$ \\
\hline $\begin{array}{l}15601 \\
15817\end{array}$ & $\frac{2}{5}$ & $\begin{array}{r}3713,6602,5355,1942,1 \\
125,1052,5752,6261,1\end{array}$ & 15877 & $\begin{array}{l}6 \\
5\end{array}$ & $\begin{array}{l}1526,4348,10855,7241,2955 \\
5047,10826,5920,14615,3975\end{array}$ \\
\hline $\begin{array}{l}15889 \\
15913\end{array}$ & $2 \frac{1}{5}$ & $\begin{array}{r}5039,3550,8229,12499,1046 \\
11459,10305,8302,6050,10399\end{array}$ & $\begin{array}{l}15901 \\
15937\end{array}$ & $\begin{array}{r}10 \\
7\end{array}$ & $\begin{array}{r}7481,7934,15880,11017,9111 \\
9250\end{array}$ \\
\hline 15973 & $\begin{array}{l}5 \\
7\end{array}$ & $4856,967,826,2945,5979$ & $\begin{array}{l}16033 \\
16069\end{array}$ & 5 & $7495,11842,8957,11703,7652$ \\
\hline 29 & 1 & $875,4864,16089,13435,9344$ & 16141 & 6 & $10300,8495,15501,6697,7646$ \\
\hline $\begin{array}{l}16189 \\
16273\end{array}$ & $\frac{2}{7}$ & $\begin{array}{r}8405,6140,465,15772,1 \\
15827,295,3592,933,4628\end{array}$ & $\begin{array}{l}16249 \\
16333\end{array}$ & 172 & $14037,1552,16165,10781,3452$ \\
\hline 16369 & 7 & $1409,1,1534,1053,2384$ & 16381 & 2 & $1078,10208,15975,6767,1$ \\
\hline $\begin{array}{l}16417 \\
16477\end{array}$ & $\begin{array}{r}10 \\
2\end{array}$ & $\begin{array}{l}11281,16191,6928,12104 \\
11056,7153,2219,10755,2\end{array}$ & $\begin{array}{l}16453 \\
16561\end{array}$ & $\frac{2}{7}$ & $\begin{array}{r}1856,8164,6993,14711,1 \\
, 14656,14819,5479,16112\end{array}$ \\
\hline $\begin{array}{l}16573 \\
16657\end{array}$ & $\frac{2}{5}$ & $\begin{array}{r}8201,4693,2703,1870,2 \\
938,3088,213,12755,9163\end{array}$ & $\begin{array}{l}16633 \\
16693\end{array}$ & 15 & $\begin{array}{r}5080,1337,3529,1640 \\
903,4,4823,12667,2\end{array}$ \\
\hline & 13 & $15853,7671,13667,5098,4898$ & 16741 & 2 & $8080,2537,5755,8661$ \\
\hline $\begin{array}{l}16921 \\
16993\end{array}$ & $\begin{array}{l}17 \\
10\end{array}$ & $\begin{array}{r}903,94,3907,983,12920 \\
3063,1474,6105,11467,2948\end{array}$ & $\begin{array}{l}16981 \\
17029\end{array}$ & $10^{2}$ & $\begin{array}{l}0,7629,10691,2914,1 \\
056,4064,2845,14847\end{array}$ \\
\hline 17041 & $\begin{array}{l}7 \\
2\end{array}$ & $\begin{array}{r}4750,1,16395,13481,11060 \\
5452,6038,12981,14411,1\end{array}$ & 17053 & 2 & $3249,7177,11771,4270,2$ \\
\hline 17 & & $4906,14552,12299,9219,1$ & & 14 & $12434,12448,15383,6031,11241$ \\
\hline 17257 & 5 & $\begin{array}{r}4299,6658,5209,953,7484 \\
3069,12844,14375,277,2\end{array}$ & 17293 & 7 & $7525,13666,12992,15287,9489$ \\
\hline & $1 i$ & $9255,8428,14375,9469,16856$ & & 2 & $1773,2,11843,10816,1$ \\
\hline & $\begin{array}{r}11 \\
5\end{array}$ & $\begin{array}{l}1067,1,11745, \\
77,7811,4252,8\end{array}$ & & & 11475 \\
\hline & 11 & $547,4708,2259,13199,17564$ & 17581 & 10 & $4835,7910,2661,15742,3955$ \\
\hline 49 & 2 & $\begin{array}{r}113530,4799,2727,8977,2 \\
10930,47967,2727\end{array}$ & 17761 & 19 & $15973,13648,2741,10383,17426$ \\
\hline 17871 & $\frac{1}{5}$ & $\begin{array}{r}775,2056,11807,10059,10520 \\
7107,5576,2476,6383,1\end{array}$ & $\begin{array}{l}1 \\
17989 \\
1799\end{array}$ & 2 & $2279,5380,17263,1335,16508$ \\
\hline & 6 & 6., $16703,15686,15915$ & & & $\begin{array}{r}8999,13900,7587,7273,10544 \\
3813,10132,13933,17651,14114\end{array}$ \\
\hline 69 & $\begin{array}{l}23 \\
11\end{array}$ & $\begin{array}{r}6781,4336,14120,1337,3633 \\
16143,6280,7169,14239,3140\end{array}$ & $\begin{array}{l}18133 \\
18181\end{array}$ & $\begin{array}{l}5 \\
2\end{array}$ & $\begin{array}{r}874,10671,8465,2948,1 \\
6186,14168,10931,6819,1\end{array}$ \\
\hline & 7 & $721,14410,7593,8369,10604$ & 18229 & $2_{1}^{2}$ & $533,5728,12675,2$ \\
\hline $\begin{array}{l}8253 \\
8301\end{array}$ & $\begin{array}{l}5 \\
6\end{array}$ & $\begin{array}{r}3476,14860,3359,5083,10185 \\
7141,1462,14591,10166,16839\end{array}$ & & 10 & $\begin{array}{r}2356,16841,15853,13479,14810 \\
4961,17488,17835,3763,15884\end{array}$ \\
\hline 8397 & 6 & $12139,3568,16838,3503,14829$ & 18433 & 5 & $13647,6844,9065,11245,6296$ \\
\hline 8493 & $\begin{array}{l}5 \\
2\end{array}$ & $\begin{array}{r}6388,4787,11953,3279,9572 \\
13217,3241,9441,17614,2\end{array}$ & 184817 & $\begin{array}{r}13 \\
6\end{array}$ & $\begin{array}{r}8711,8704,11371,10383,3824 \\
17864,16918,17189,1909,1599\end{array}$ \\
\hline 18541 & 6 & $\begin{array}{r}11845,10774,2249,5228,7767 \\
14335,9087,18418,17093,2\end{array}$ & 18553 & $\begin{array}{r}5 \\
10\end{array}$ & $14653,10852,10793,1425,15734$ \\
\hline & 2 & $2783,1792,10100,6633,1$ & 18 & ${ }^{1}$ & $2691,9521,18226,6482,1$ \\
\hline 18973 & $\begin{array}{l}5 \\
2\end{array}$ & $\begin{array}{r}6369,358,14327,92,1 \\
9700,380,14237,2937,1\end{array}$ & $\begin{array}{l}18913 \\
19009\end{array}$ & 23 & $\begin{array}{r}7823,9352,1963,14157,9290 \\
2763,11044,11191,5981,1190\end{array}$ \\
\hline
\end{tabular}


Table 10: Table of $\gamma_{1}, \ldots, \gamma_{5}$ for $13-$ GDD $13^{q}$ construction (cont.)

\begin{tabular}{|c|c|c|c|c|c|}
\hline & $\omega$ & $\gamma_{1}, \ldots, \gamma_{5}$ & $q$ & $\omega$ & $\gamma_{1}, \ldots, \gamma_{5}$ \\
\hline $\begin{array}{l}19069 \\
19141\end{array}$ & $\begin{array}{l}2 \\
2\end{array}$ & $\begin{array}{r}13649,13610,2746,17367,1 \\
10484,1913,4612,18117,1\end{array}$ & $\begin{array}{l}19081 \\
19213\end{array}$ & $\begin{array}{r}17 \\
5\end{array}$ & $17378, \begin{array}{r}10906,4331,18937,10737 \\
3860,1,12340,1841,10821\end{array}$ \\
\hline $\begin{array}{l}1914 \\
19237\end{array}$ & $\frac{2}{2}$ & $6009,17590,2858,2675,1$ & $\begin{array}{l}19213 \\
19249\end{array}$ & 7 & $12475,5728,17927,16701,5222$ \\
\hline 19321 & 5 & $8091,5740,6007,17573,140$ & $\begin{array}{l}19309 \\
19333\end{array}$ & $\begin{array}{l}6 \\
2\end{array}$ & $\begin{array}{r}13244,12454,3859,7475,15423 \\
15957,13490,12514,13547,1\end{array}$ \\
\hline $\begin{array}{l}19381 \\
19429\end{array}$ & $\begin{array}{l}7 \\
6\end{array}$ & $\begin{array}{l}19346,5315,19054,12451,5463 \\
18817,11350,3272,15335,8079\end{array}$ & $\begin{array}{l}19417 \\
19441\end{array}$ & $\begin{array}{r}5 \\
13\end{array}$ & $\begin{array}{r}16322,4281,10588,11411,1 \\
12489,1,784,15377,11894\end{array}$ \\
\hline $\begin{array}{l}19477 \\
19501\end{array}$ & $\begin{array}{l}6 \\
2\end{array}$ & $\begin{array}{r}14753,12616,3817,16850,6861 \\
9488,10853,3508,18699\end{array}$ & 19489 & 19 & $12171,7666,6565,14957,14276$ \\
\hline 19609 & 13 & $11593,13265,6591,8716,3680$ & 19681 & 11 & $10547,7420,2911,17169,17444$ \\
\hline $\begin{array}{l}19717 \\
19777\end{array}$ & 11 & $\begin{array}{r}1961,2,5871,2266,1 \\
15206,1,5944,17477,8325\end{array}$ & $\begin{array}{l}19753 \\
19801\end{array}$ & 13 & $\begin{array}{r}16875,11984,5483,12040,1 \\
9463,19775,15298,1509,15560\end{array}$ \\
\hline $\begin{array}{l}19813 \\
19993\end{array}$ & 10 & $\begin{array}{r}5219,67,15555,8620,2 \\
11195,9028,6367,13971,18056\end{array}$ & $\begin{array}{l}19861 \\
20029\end{array}$ & ${ }_{2}^{11}$ & $\begin{array}{l}5533,8045,3548,13768,5073 \\
19931,18508,12998,1059,1\end{array}$ \\
\hline 20089 & $\begin{array}{l}7 \\
10\end{array}$ & $\begin{array}{l}10737,, 17386,18475,9197,9188 \\
11595,11512,5231,14893,2912\end{array}$ & 20101 & $\underset{2}{6}$ & $\begin{array}{l}14444,3382,14987,4069,16719 \\
10792,17043,8918,19313,2209\end{array}$ \\
\hline $\begin{array}{l}20113 \\
20161 \\
20233\end{array}$ & $\begin{array}{l}10 \\
13 \\
5\end{array}$ & $16571,18412,8161,16713,19286$ & $\begin{array}{l}20149 \\
20173 \\
20269\end{array}$ & $\frac{2}{2}$ & $\begin{array}{r}10792,17043,8918,19313,2209 \\
6226,8593,5195,19797,2\end{array}$ \\
\hline $\begin{array}{l}20341 \\
20389\end{array}$ & $\begin{array}{l}0 \\
2 \\
6\end{array}$ & $\begin{array}{r}18440,13773,9533,19468,1 \\
10556,13589,7912,2197,14961\end{array}$ & $\begin{array}{l}20353 \\
20509\end{array}$ & $\begin{array}{l}5 \\
2\end{array}$ & $9981,18646,17479,14681,15032$ \\
\hline $\begin{array}{l}20521 \\
20593\end{array}$ & 11 & $\begin{array}{r}7971,1582,7721,9655,9176 \\
18965,8690,19372,19797,13255\end{array}$ & $\begin{array}{l}20533 \\
20641\end{array}$ & $\frac{2}{7}$ & $\begin{array}{r}8788,835,15423,3677,2 \\
1533,1,3977,10546,11768\end{array}$ \\
\hline $\begin{array}{l}20749 \\
20809\end{array}$ & $\begin{array}{l}2 \\
7\end{array}$ & $\begin{array}{r}15429,4,12659,14161,2 \\
895,8242,339,7949,9512\end{array}$ & $\begin{array}{l}20773 \\
20857\end{array}$ & $\begin{array}{r}2 \\
10\end{array}$ & $\begin{array}{r}5219,17143,4719,14920,2 \\
1,19276,10901,1561,17696\end{array}$ \\
\hline $\begin{array}{l}20929 \\
21013\end{array}$ & $\begin{array}{l}7 \\
2\end{array}$ & $\begin{array}{r}14727,17182,17905,683,12710 \\
19089,19526,20782,8021,1\end{array}$ & $\begin{array}{l}21001 \\
21061\end{array}$ & $\begin{array}{r}11 \\
7\end{array}$ & $\begin{array}{r}1945,15670,13751,5631,8192 \\
16316,15310,13991,9589 \\
18045\end{array}$ \\
\hline $\begin{array}{l}21121 \\
21169\end{array}$ & $\begin{array}{l}19 \\
13\end{array}$ & $\begin{array}{r}19571,3412,6211,11907,1706 \\
11236,1,6791,16365,18290\end{array}$ & $\begin{array}{l}21157 \\
21193\end{array}$ & $\begin{array}{r}2 \\
11\end{array}$ & $\begin{array}{r}15061,6135,11818,2837,2 \\
17785,20638,9107,392,15081\end{array}$ \\
\hline $\begin{array}{l}21277 \\
21397\end{array}$ & $\begin{array}{r}6 \\
2\end{array}$ & $\begin{array}{c}1490,7174,1577,8503,14103 \\
21159,985,1847,15532,2\end{array}$ & $\begin{array}{l}21313 \\
21433\end{array}$ & $\begin{array}{r}1 \\
5 \\
5\end{array}$ & $\begin{array}{r}13545,11299,19138,14795,10700 \\
21021,10544,11110,5759,1\end{array}$ \\
\hline $\begin{array}{l}21481 \\
21517\end{array}$ & 13 & $\begin{array}{r}9911,9742,7923,17846,1 \\
9233,5829,12112,10406,1\end{array}$ & $\begin{array}{l}21493 \\
21529\end{array}$ & $\begin{array}{r}2 \\
11\end{array}$ & $\begin{array}{r}15766,5720,10683,4355,1 \\
12809,10156,9633,10915,1886\end{array}$ \\
\hline $\begin{array}{l}21577 \\
21601\end{array}$ & $\begin{array}{l}5 \\
7\end{array}$ & $\begin{array}{r}298,19790,19593,8915,18757 \\
19205,4060,8383,8553,8120\end{array}$ & $\begin{array}{l}21589 \\
21613\end{array}$ & $\begin{array}{r}1 \\
2 \\
2\end{array}$ & $\begin{array}{r}829,21255,7756,15785,2 \\
8770,15104,10883,11235,1\end{array}$ \\
\hline 49 & 14 & $13345,19786,15147,2591,17924$ & 21661 & $\frac{2}{2}$ & $13289,2,13576,6999,1$ \\
\hline 17 & 7 & $\begin{array}{l}3987,19114,809,21313,14456 \\
557,20132,7105,17578,16629\end{array}$ & 21841 & 11 & $\begin{array}{l}12089,5368,20198,3057,14581 \\
18833,20860,8331,5809,19880\end{array}$ \\
\hline 37 & $\begin{array}{l}7 \\
7\end{array}$ & $\begin{array}{l}17663,14936,907,16342,8325 \\
13232,3982,3499,6551,11559\end{array}$ & $\begin{array}{l}21961 \\
22093\end{array}$ & $\begin{array}{r}17 \\
6\end{array}$ & $\begin{array}{r}6569,11968,10807,7827,18182 \\
7373,13552,6302,15181,8541\end{array}$ \\
\hline 22129 & 19 & $7777,18921,6004,2627,4874$ & 22153 & 5 & $4061,3850,12549,7093,15458$ \\
\hline $\begin{array}{l}22189 \\
22273\end{array}$ & $\frac{2}{5}$ & $\begin{array}{r}21467,7724,1672,8487,1 \\
7805,10099,4138,22029,596\end{array}$ & $\begin{array}{l}22201 \\
22369\end{array}$ & & $\begin{array}{l}14560,11249,20114,20571,1 \\
5089,1366,18023,1239,17672\end{array}$ \\
\hline $\begin{array}{l}22381 \\
22453\end{array}$ & $\begin{array}{l}5 \\
10 \\
5\end{array}$ & $\begin{array}{r}5569,11956,11063,873,6356 \\
18431,5140,21008,22225,4383\end{array}$ & $\begin{array}{l}22409 \\
22501\end{array}$ & $\begin{array}{l}11 \\
14\end{array}$ & $10687,18459,4907,7204,1718$ \\
\hline 22 & $\begin{array}{l}\breve{2} \\
2\end{array}$ & $4331,2,11266,14253,1$ & 22573 & 6 & $22438,11153,13563,6103,11420$ \\
\hline 21 & $\frac{2}{2}$ & $\begin{array}{r}22301,4522,5852,16743,1 \\
16601,1621,3549,5374,2\end{array}$ & 22741 & $\frac{2}{7}$ & $\begin{array}{r}20743,11458,11573,1875,2 \\
8266,16178,3989,943,22137\end{array}$ \\
\hline 22777 & 7 & $19168,1393,15017,12014,6021$ & 22801 & & $4099,11704,3467,5409,6080$ \\
\hline $\begin{array}{l}22861 \\
22993\end{array}$ & 2 & $\begin{array}{r}3814,21869,4903,747,2 \\
377,18682,8511,12539,17906\end{array}$ & $\begin{array}{l}22921 \\
23017\end{array}$ & 7 & $\begin{array}{r}673,18076,8381,7267,17522 \\
5391,21578,16444,6623,1\end{array}$ \\
\hline 29 & 2 & $\begin{array}{r}8474,243,19613,16468,1 \\
17314,15769,5189,7671,2\end{array}$ & 23041 & $1 \frac{1}{5}$ & $15395,9202,19899,703,19196$ \\
\hline 23 & 2 & $\begin{array}{l}17314,15769,5189,7671,2 \\
7847,489,21439,10162,2\end{array}$ & 23209 & 31 & $1400,21868,19367,7681,4629$ \\
\hline $\begin{array}{l}23 \\
23\end{array}$ & 6 & $\begin{array}{r}2451,1018,21259,7682,22251 \\
8302,8873,14384,10071,1069\end{array}$ & $\begin{array}{l}23293 \\
23497\end{array}$ & 5 & $\begin{array}{l}49,22196,19234,3035,5295 \\
53,1162,16109,11719,5216\end{array}$ \\
\hline $\begin{array}{l}23509 \\
23581\end{array}$ & $\overline{6}$ & $\begin{array}{r}5279,3712,18074,4857,1 \\
21805,22840,13424,1727,741\end{array}$ & 23557 & 5 & $4846,1220,19029,16667,7423$ \\
\hline $\begin{array}{l}23581 \\
23629 \\
23689\end{array}$ & 2 & $20212,19670,737,7089,1$ & 23677 & 5 & $4415,9142,12349,7310,4185$ \\
\hline $\begin{array}{l}23689 \\
23773\end{array}$ & $1 \frac{1}{5}$ & $\begin{array}{r}22135,11572,18041,18513,5786 \\
23465,21261,23738,17752,1\end{array}$ & $\begin{array}{l}23761 \\
23833\end{array}$ & $\begin{array}{l}7 \\
5\end{array}$ & $\begin{array}{r}917,17620,18847,9939,10520 \\
5848,2325,3155,5042,10393\end{array}$ \\
\hline $\begin{array}{l}23857 \\
23893\end{array}$ & $\begin{array}{l}5 \\
5\end{array}$ & & $\begin{array}{l}23869 \\
23917\end{array}$ & $\frac{2}{2}$ & $16528,7595,17834,15105,2707$ \\
\hline 23929 & 7 & $23177,12862,4137,9913,7520$ & $\begin{array}{l}23916 \\
23977\end{array}$ & 2 & $\begin{array}{l}0,2,17434 \\
4822,1007\end{array}$ \\
\hline$\frac{24}{24}$ & $\begin{array}{l}14 \\
10\end{array}$ & $\begin{array}{r}10693,7492,2913,12335,7892 \\
2239,7622,2944,12593,2283\end{array}$ & $\begin{array}{l}24049 \\
24097\end{array}$ & 19 & $\begin{array}{r}10033,5878,2757,5993,5984 \\
1489,23188,14493,11825,3206\end{array}$ \\
\hline & 2 & $\begin{array}{r}15250,19773,8923,19367,2 \\
9854\end{array}$ & 24121 & (5 & $9292,19287,4825,9347,6038$ \\
\hline 24181 & 17 & $\begin{array}{r}9854,2344,15437,3601,21789 \\
4159,17026,17135,18608,5319\end{array}$ & & $1 \frac{1}{2}$ & $\begin{array}{l}17881,2710,1088999218,19485 \\
154,977,19070,4599,1\end{array}$ \\
\hline & $\begin{array}{l}5 \\
7\end{array}$ & $\begin{array}{l}20690,21209,8493,16090,12877 \\
10826,20561,5932,14617,22041\end{array}$ & $\begin{array}{l}24373 \\
24469\end{array}$ & 14 & $\begin{array}{r}15205,6071,6506,23170,23643 \\
9991,22360,8366,155,4557\end{array}$ \\
\hline & 11 & $2843,9748,20497,10821,15842$ & 24517 & $\begin{array}{r}14 \\
5\end{array}$ & $7081,21448,10655,8882,22425$ \\
\hline $\begin{array}{l}24649 \\
24709\end{array}$ & 11 & $10957,2212,14399,10251,1106$ & $\begin{array}{l}24697 \\
24733\end{array}$ & $\begin{array}{l}5 \\
5 \\
2\end{array}$ & $2223,7667,727,21388,15332$ \\
\hline 247 & 2 & $22445,2,526,19565,1$ & 24793 & 5 & $\begin{array}{r}1594,12193,2085,12785,2 \\
16743,4088,155,4936,1\end{array}$ \\
\hline & 14 & 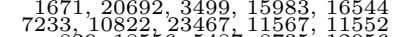 & 877 & 5 & $\begin{array}{l}347,9761,7790,1 \\
191,24659,11612\end{array}$ \\
\hline$\overline{2} 5153$ & $\begin{array}{r}5 \\
10\end{array}$ & $\begin{array}{r}829,18556,5487,8735,12056 \\
145,24922,23259,23987,15062\end{array}$ & 25189 & $\begin{array}{r}5 \\
2\end{array}$ & $\begin{array}{r}1145,21746,8686,3801,2899 \\
9609,23740,18320,10265,1\end{array}$ \\
\hline $\begin{array}{l}\overline{2} 5237 \\
25309\end{array}$ & $\stackrel{2}{13}$ & $\begin{array}{r}14853,2848,22229,147779,2 \\
21256,20054,12767,11235,1369\end{array}$ & & 19 & $\begin{array}{l}21826,9092,4553,14551 \\
1169,14548,15061,7245\end{array}$ \\
\hline & $\begin{array}{ll}10 \\
10\end{array}$ & $\begin{array}{r}23795,18664,20474,4911,1 \\
17443,11104,4061,10071,18320\end{array}$ & $\begin{array}{l}25453 \\
25561\end{array}$ & $\begin{array}{l}19 \\
11\end{array}$ & $\begin{array}{r}9491,24296,11914,2349 \\
7351,5992,6975,12671,299\end{array}$ \\
\hline & 7 & $13495,15892,8255,19041,20750$ & 2 & 10 & $14599,6478,5435,23924,4815$ \\
\hline & $\begin{array}{l}5 \\
2\end{array}$ & $\begin{array}{r}7259,15532,19773,5605,13544 \\
4173,23828,18058,7319,1\end{array}$ & & $\begin{array}{l}5 \\
2\end{array}$ & $\begin{array}{r}11699,21400,6524,5781,1 \\
6893,4154,9297,8446,1\end{array}$ \\
\hline & 7 & & & $\begin{array}{r}\overline{7} \\
10\end{array}$ & $\begin{array}{l}1,10264,2661,16388 \\
10,24911,18751,548\end{array}$ \\
\hline & 2 & $\begin{array}{r}12343,25660,3303,21233,19568 \\
20561,2,16036,12867,1\end{array}$ & 25969 & 7 & \\
\hline & 11 & $\begin{array}{r}5972,1,16408,11861,1203 \\
25939,15934,1832,13961,10095\end{array}$ & & $\begin{array}{r}5 \\
13 \\
\end{array}$ & $\begin{array}{r}15062,5499,20975,12244,1 \\
25093,21586,21029,23367,6356\end{array}$ \\
\hline & 13 & $\begin{array}{r}23097,4256,11903,13126,1 \\
8559,1,24298,3785,20210\end{array}$ & $\begin{array}{l}26113 \\
26209\end{array}$ & ${ }_{11}^{7}$ & $\begin{array}{r}6791,1,9016,18507,404 \\
25360,21437,20815,23402\end{array}$ \\
\hline & $\begin{array}{l}10 \\
6\end{array}$ & $13061,1546,5869,13670,24747$ & 26317 & & $22331,22060,5078,15565,4257$ \\
\hline & $x_{*}^{0}$ & $\begin{array}{r}1092 \\
899\end{array}$ & & 2 & $1293,7102,18476,16001$, \\
\hline & & $\begin{array}{l}4245,9184,12937,22949,17876 \\
3361,15712,16814,4733,14385\end{array}$ & 26 & 10 & 12861 \\
\hline 37 & 10 & $19453,22966,8573,3081,19196$ & & 10 & 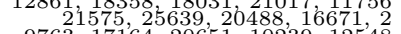 \\
\hline 33 & $\begin{array}{l}5 \\
5\end{array}$ & $\begin{array}{l}5338,5486,19187,9531,1 \\
25828,1881,671,23360,1\end{array}$ & & ${ }^{11}$ & $\begin{array}{r}9763,17164,20651,10239,12548 \\
14146,5420,2917,15251,6117\end{array}$ \\
\hline & 2 & $\begin{array}{r}2002829,2,16396,8465,1 \\
2829\end{array}$ & & 5 & $18143,8692,17277,16285,11402$ \\
\hline 27 & 2 & $\begin{array}{r}18932,982,21243,9589,8577 \\
17019,22813,665,6724,2\end{array}$ & 27 & $\begin{array}{r}7 \\
6 \\
\end{array}$ & $6623,2284,15829,19112,165800$ \\
\hline $\begin{array}{l}27 \\
27\end{array}$ & $\begin{array}{l}5 \\
2\end{array}$ & $22195,12754,23943,20915,25508$ & $\begin{array}{l}27361 \\
27409\end{array}$ & $\begin{array}{r}7 \\
13\end{array}$ & $\begin{array}{r}12112,1,6167,19299,21146 \\
5555,22234,9945,8101,15722\end{array}$ \\
\hline & 7 & $12082,668,7277,8671$ & 年 & 19 & $27445,13287,17672$ \\
\hline 29 & 11 & $\begin{array}{r}14577,17620,7237,5253,8462 \\
24494,26026,23657,727,9219\end{array}$ & $\begin{array}{l}27541 \\
27697\end{array}$ & & $\begin{array}{r}5741,10959,22603,11308,7064 \\
10670,9208,12615,10295,1\end{array}$ \\
\hline & 2 & $17259,18860,8110,425,1$ & & & $25881,2662,5483,15817,9224$ \\
\hline 27 & $5_{2}^{5}$ & $\begin{array}{r}19604,9784,24065,82 \\
17453,2,21130,115\end{array}$ & 27 & & $14234,27508,83,1209$ \\
\hline $901-2-2$ & 5 & $\begin{array}{r}17453,2,21130,11511,1 \\
27016,16556,14647,14225,4731\end{array}$ & 280 & $\begin{array}{r}13 \\
5\end{array}$ & $\begin{array}{l}828699,13736,17739,1 \\
6246,1373,17\end{array}$ \\
\hline & & $24575,7209,22471,19060,74$ & & 19 & $1,7097,2$ \\
\hline
\end{tabular}


Table 10: Table of $\gamma_{1}, \ldots, \gamma_{5}$ for $13-$ GDD $13^{q}$ construction (cont.)

\begin{tabular}{|c|c|c|c|c|c|}
\hline$q$ & $\omega$ & $\gamma_{1}, \ldots, \gamma_{5}$ & & $\omega$ & $\gamma_{1}, \ldots, \gamma_{5}$ \\
\hline 28201 & $\begin{array}{r}11 \\
2\end{array}$ & $\begin{array}{r}13367,11596,23349,4357,5342 \\
16095,20812,21086,623,1\end{array}$ & $\begin{array}{l}28297 \\
28393\end{array}$ & $\begin{array}{r}5 \\
15\end{array}$ & $\begin{array}{r}28211,140,2715,13030,1 \\
1262,1540,16699,22511\end{array}$ \\
\hline $\begin{array}{l}28429 \\
28513\end{array}$ & $\overline{2}$ & $9717,21700,5665,1889,22136$ & $\begin{array}{l}28477 \\
28537\end{array}$ & $\stackrel{2}{5}$ & $6430,27338,24225,21203,27301$ \\
\hline $\begin{array}{l}28549 \\
28573\end{array}$ & $\begin{array}{r}2 \\
2\end{array}$ & $\begin{array}{r}3335,2,892,21501,1 \\
11817,28297,13349,28258,2\end{array}$ & $\begin{array}{l}28561 \\
28597\end{array}$ & $\begin{array}{l}* \\
2\end{array}$ & $22209,8311,8512,13451,11900$ \\
\hline $\begin{array}{l}28621 \\
28669\end{array}$ & $\begin{array}{r}13 \\
6\end{array}$ & $\begin{array}{r}14416,17455,2303,22214,4065 \\
4087,22144,15620,845,6525\end{array}$ & $\begin{array}{l}28657 \\
28729\end{array}$ & $\begin{array}{r}5 \\
22\end{array}$ & $\begin{array}{r}8913,5584,13789,5741,27932 \\
9007,23044,485,7341,11522\end{array}$ \\
\hline $\begin{array}{l}28753 \\
28813\end{array}$ & $\begin{array}{r}10 \\
2\end{array}$ & $\begin{array}{r}12919,21868,12969,14477,24272 \\
12927,13825,24748,7733,2\end{array}$ & $\begin{array}{l}28789 \\
28837\end{array}$ & $\begin{array}{r}7 \\
2\end{array}$ & 4907, $23095,17014,19190,16149$ \\
\hline $\begin{array}{l}28909 \\
28933 \\
29077\end{array}$ & $\begin{array}{l}2 \\
2 \\
2\end{array}$ & $\begin{array}{r}2573,3,27338,11728,26773 \\
24923,26564,4918,12813,1 \\
19209,11452,6938,24941,1\end{array}$ & $\begin{array}{l}28921 \\
29017 \\
29101\end{array}$ & $\begin{array}{r}11 \\
5 \\
2\end{array}$ & $\begin{array}{r}19989,123266,20291,4856 \\
7617,6880,28430,22415,1 \\
6430,9671\end{array}$ \\
\hline $\begin{array}{l}29137 \\
29209\end{array}$ & $\begin{array}{l}5 \\
7\end{array}$ & $\begin{array}{r}21095,4360,15079,27525,2180 \\
17615,21484,12177,29047,15704\end{array}$ & $\begin{array}{l}29173 \\
29221\end{array}$ & $\frac{1}{2}$ & $21439,22409,26469,15046,2$ \\
\hline $\begin{array}{l}29269 \\
29401\end{array}$ & $\begin{array}{r}6 \\
13\end{array}$ & $\begin{array}{l}20483,9472,12409,20396,18399 \\
25797,25006,1729,29375,27890\end{array}$ & $\begin{array}{l}29389 \\
29437\end{array}$ & $\frac{2}{2}$ & $\begin{array}{r}2871,2,8668,28229,1 \\
12663,9188,15724,22751,1\end{array}$ \\
\hline 73 & 5 & $23759,16383,19246,3793,15812$ & $\begin{array}{l}29569 \\
29629\end{array}$ & 17 & $6777,1,3820,28271,27170$ \\
\hline 41 & $\begin{array}{r}10 \\
7 \\
5\end{array}$ & $7449,3436,9199,6869,1718$ & 29761 & 17 & $49,12880,8629,22449,22244$ \\
\hline $\begin{array}{l}3 . \\
17 \\
80\end{array}$ & 2 & $\begin{array}{l}821,18964,9687,11215,14360 \\
22791,25840,28208,29255,1\end{array}$ & 29881 & 7 & $\begin{array}{l}9751,5170,8475,12425,4586 \\
25288,15137,11079,17276,1\end{array}$ \\
\hline $\begin{array}{l}29989 \\
30097\end{array}$ & $10^{2}$ & $\begin{array}{r}13541,6793,10072,5289,2 \\
11347,10612,12525,965,12164\end{array}$ & $\begin{array}{l}30013 \\
30109\end{array}$ & 2 & $\begin{array}{rl}10547,3 & 15572,22498,1 \\
14104,7946,19805,24459,1\end{array}$ \\
\hline $\begin{array}{l}30133 \\
30181 \\
30253\end{array}$ & $\begin{array}{l}5 \\
2 \\
2\end{array}$ & $\begin{array}{l}6330,27092,21113,9553,15627 \\
21392,3646,22509,28229\end{array}$ & $\begin{array}{l}30169 \\
30241 \\
30313\end{array}$ & $1 \frac{7}{5}$ & $\begin{array}{l}4853,4732,6615,11209,17450 \\
1901,20944,8965,18201,10472\end{array}$ \\
\hline $\begin{array}{l}30469 \\
30517\end{array}$ & $\frac{2}{2}$ & $\begin{array}{l}23643,7562, \\
3689\end{array} 24430,20612,15633,1$ & $\begin{array}{l}30493 \\
30529\end{array}$ & $\begin{array}{r}6 \\
13\end{array}$ & $\begin{array}{r}1192,27863,17349,21541,2630 \\
6719,11224,9301,30501,5612\end{array}$ \\
\hline $\begin{array}{l}30553 \\
30637\end{array}$ & $\begin{array}{l}5 \\
2\end{array}$ & $\begin{array}{r}9611,27868,10927,4047,13934 \\
30603,11954,1186,21761,1\end{array}$ & $\begin{array}{l}30577 \\
30649\end{array}$ & $\begin{array}{r}5 \\
7\end{array}$ & $\begin{array}{r}20411,4558,17540,18195,1 \\
13197,11572,7817,29131,25778\end{array}$ \\
\hline $\begin{array}{l}30661 \\
30757\end{array}$ & $\overline{2}$ & $\begin{array}{r}4270,17066,9209,29499,1 \\
5993,25480,2480,27763,24123\end{array}$ & $\begin{array}{l}30697 \\
30781\end{array}$ & $\begin{array}{r}10 \\
2\end{array}$ & $\begin{array}{r}7021,19762,7797,3047,18512 \\
6045,15046,16910,10109,1\end{array}$ \\
\hline $\begin{array}{l}30817 \\
30841\end{array}$ & $\begin{array}{r}5 \\
7\end{array}$ & $\begin{array}{r}6963,28456,10261,21785,14228 \\
13439,14338,4687,7815,3944\end{array}$ & $\begin{array}{l}30829 \\
30853\end{array}$ & $\begin{array}{r}2 \\
2\end{array}$ & $\begin{array}{r}22779,4270,7466,8507,1 \\
30791,3490,5594,6843,1\end{array}$ \\
\hline $\begin{array}{l}937 \\
033\end{array}$ & $\begin{array}{l}15 \\
10\end{array}$ & $313,20056,4562,3353,29109$ & $\begin{array}{l}30949 \\
31069\end{array}$ & 10 & $\begin{array}{l}27802,18107,3361,9441 \\
6983,2,18261,14350,1\end{array}$ \\
\hline 81 & $\begin{array}{r}13 \\
7\end{array}$ & $\begin{array}{r}4443,22708,13103,21883,15518 \\
21988,10100,13697,13873,15603\end{array}$ & $\begin{array}{l}31153 \\
31189\end{array}$ & $\begin{array}{l}10 \\
13\end{array}$ & $\begin{aligned} 24185 & , 171 \\
22541 & \end{aligned}$ \\
\hline 31321 & $\begin{array}{l}6 \\
7\end{array}$ & $\begin{array}{r}28064,16054,17845,18425,15183 \\
3167,24628,28611,12949,12314\end{array}$ & $\begin{array}{l}31249 \\
31333\end{array}$ & 23 & $\begin{array}{r}26105,3933,28906,26863,20 \\
23639,30956,12220,19825,11637\end{array}$ \\
\hline $\begin{array}{l}357 \\
477\end{array}$ & 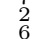 & $\begin{array}{r}15443,6032,16455,25198,1 \\
26993,6286,28573,26468,25191\end{array}$ & 313903 & ב & $3,1259,28263,15572$ \\
\hline & 5 & $\begin{array}{r}25885,31180,3497,8625,30848 \\
13191,8804,707,1978,1\end{array}$ & 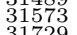 & 7 & $9538,29054,8201,6283,22119$ \\
\hline & 6 & $28021,30268,1763,29588,1473$ & 31849 & 14 & $\begin{array}{r}897,6796,3763,9119,21548 \\
89,30238,3727,1923,2618\end{array}$ \\
\hline 81 & $\begin{array}{r}11 \\
6\end{array}$ & $\begin{array}{r}28127,5260,23744,11419,18861 \\
28342,5469,22009,17267,8270\end{array}$ & $\begin{array}{l}31957 \\
32029\end{array}$ & & $\begin{array}{r}10511,30662,30993,3190,1 \\
20933,20672,28581,28660,13717\end{array}$ \\
\hline $\begin{array}{l}32041 \\
32089\end{array}$ & 13 & $\begin{array}{r}17327,23373,18572,9946,1 \\
23181,18292,9863,22795,344\end{array}$ & 32173 & $\frac{2}{5}$ & $\begin{array}{l}26458,31963,1937,3459,2 \\
11794,18128,23507,28653\end{array}$ \\
\hline & $\begin{array}{r}5 \\
2\end{array}$ & $3971,25413,21286,27373,21944$ & $\begin{array}{l}32257 \\
32353\end{array}$ & $\begin{array}{l}15 \\
15\end{array}$ & $\begin{array}{l}21259,15754,28755,31871,20084 \\
17032,31364,4663,29327,495\end{array}$ \\
\hline & 5 & $\begin{array}{r}987,23134,21325,20045,14768 \\
23918,15232,24649,28229,21225\end{array}$ & 101 & 7 & $28403,10090,20091,16699,20180$ \\
\hline & $\begin{array}{l}5 \\
2\end{array}$ & 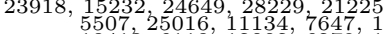 & $\begin{array}{l}32497 \\
32569\end{array}$ & & 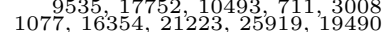 \\
\hline & 0 & $18413,6118,12002$, & & 5 & 18577 \\
\hline & & $19559,2,5896$, & & & 1345 \\
\hline
\end{tabular}

Table 11: Table of $m, w, c, c^{\prime}, c^{\prime \prime}$ for $\operatorname{BiBD}(q, 12,1)$ construction ( $q$ prime)

\begin{tabular}{|c|c|c|c|c|c|c|c|c|c|c|c|}
\hline$q$ & $m$ & $w$ & $\mathrm{C}$ & $\mathrm{c}^{\prime}$ & $\mathrm{C}^{\prime \prime}$ & $q$ & $m$ & $w$ & C & $\mathrm{c}^{\prime}$ & C" \\
\hline $\begin{array}{r}5413 \\
6337 \\
6733 \\
7129 \\
7789 \\
8317 \\
8713 \\
9241 \\
9901 \\
10957 \\
11617 \\
12409 \\
13597 \\
14389 \\
15313 \\
16369 \\
17029 \\
18217 \\
19009 \\
19273 \\
20593 \\
21121 \\
21649 \\
22573 \\
23629 \\
23893 \\
25609 \\
25873 \\
27457 \\
28909 \\
29437 \\
29833 \\
30493 \\
31153 \\
32341\end{array}$ & $\begin{array}{r}2117 \\
1735 \\
6378 \\
2795 \\
3822 \\
7077 \\
5904 \\
7962 \\
6181 \\
8942 \\
10190 \\
4841 \\
12373 \\
7105 \\
8635 \\
13275 \\
8861 \\
17856 \\
7663 \\
14875 \\
13610 \\
11634 \\
5358 \\
15853 \\
11971 \\
2208 \\
22883 \\
21339 \\
16675 \\
2499 \\
14250 \\
15989 \\
9170 \\
26022 \\
22315\end{array}$ & $\begin{array}{r}1224 \\
4824 \\
6113 \\
5879 \\
233 \\
1286 \\
6175 \\
9074 \\
99 \\
3145 \\
9023 \\
9597 \\
4429 \\
13956 \\
6819 \\
338 \\
5519 \\
8397 \\
1625 \\
17533 \\
143 \\
13952 \\
14609 \\
688 \\
20558 \\
5681 \\
11961 \\
23045 \\
23868 \\
10917 \\
21777 \\
22037 \\
21936 \\
30976 \\
17796\end{array}$ & $\begin{array}{r}3380 \\
4184 \\
3475 \\
2230 \\
6811 \\
2936 \\
1471 \\
8415 \\
64 \\
4014 \\
7271 \\
9833 \\
5256 \\
16 \\
6148 \\
11785 \\
2782 \\
343 \\
529 \\
15625 \\
1520 \\
1704 \\
6130 \\
1296 \\
2048 \\
10368 \\
22243 \\
855 \\
27290 \\
512 \\
625 \\
15006 \\
3104 \\
2\end{array}$ & $\begin{array}{r}1941 \\
4073 \\
4630 \\
5669 \\
131 \\
7191 \\
4408 \\
5595 \\
2204 \\
7721 \\
8981 \\
5210 \\
10396 \\
12102 \\
8085 \\
9786 \\
757 \\
8847 \\
7438 \\
17315 \\
14365 \\
16841 \\
19529 \\
4175 \\
9757 \\
6725 \\
24951 \\
1200 \\
21883 \\
25493 \\
24758 \\
10215 \\
13583 \\
13202 \\
19646\end{array}$ & $\begin{array}{r}4205 \\
3621 \\
4821 \\
11 \\
6852 \\
4486 \\
4881 \\
1745 \\
6095 \\
10310 \\
5420 \\
3068 \\
2739 \\
5801 \\
875 \\
6177 \\
9953 \\
9126 \\
4112 \\
587 \\
3129 \\
15698 \\
16194 \\
14805 \\
15004 \\
23602 \\
5228 \\
4209 \\
23299 \\
24225 \\
27785 \\
8611 \\
2737 \\
12205 \\
6292\end{array}$ & $\begin{array}{r}6073 \\
6469 \\
6997 \\
7393 \\
8053 \\
8581 \\
9109 \\
9769 \\
10429 \\
11353 \\
12277 \\
12541 \\
13729 \\
14653 \\
15973 \\
16633 \\
17293 \\
18481 \\
19141 \\
19801 \\
20857 \\
21517 \\
22441 \\
23497 \\
23761 \\
24421 \\
25741 \\
27061 \\
28513 \\
29173 \\
29569 \\
30097 \\
30757 \\
32077\end{array}$ & $\begin{array}{r}2550 \\
2392 \\
2492 \\
5394 \\
6744 \\
6263 \\
5770 \\
4461 \\
6208 \\
8040 \\
7847 \\
11585 \\
5476 \\
3546 \\
12061 \\
752 \\
7309 \\
16454 \\
2425 \\
4142 \\
8688 \\
4770 \\
559 \\
14115 \\
16962 \\
15074 \\
15895 \\
26910 \\
463 \\
22565 \\
614 \\
15065 \\
10533 \\
24294\end{array}$ & $\begin{array}{r}1842 \\
4992 \\
4088 \\
1717 \\
3497 \\
424 \\
3120 \\
9507 \\
2414 \\
2307 \\
11877 \\
10157 \\
7019 \\
3693 \\
1034 \\
4010 \\
17161 \\
14188 \\
10002 \\
2184 \\
2047 \\
2190 \\
20414 \\
23231 \\
14985 \\
55662 \\
24210 \\
26896 \\
12370 \\
9972 \\
13058 \\
22771 \\
15146 \\
15305\end{array}$ & $\begin{array}{r}5141 \\
64 \\
2164 \\
4266 \\
7598 \\
398 \\
5282 \\
1557 \\
1751 \\
413 \\
8192 \\
11102 \\
10912 \\
7641 \\
4771 \\
2100 \\
10772 \\
5522 \\
128 \\
9251 \\
10 \\
18424 \\
17196 \\
10478 \\
16122 \\
19965 \\
12965 \\
256 \\
15625 \\
4 \\
289 \\
9709 \\
16766 \\
512\end{array}$ & $\begin{array}{r}4875 \\
6212 \\
1472 \\
6499 \\
3995 \\
318 \\
458 \\
324 \\
8331 \\
10442 \\
6044 \\
7160 \\
11558 \\
9119 \\
6347 \\
7929 \\
11099 \\
7092 \\
7791 \\
19613 \\
16830 \\
2063 \\
5967 \\
21327 \\
19830 \\
17054 \\
17830 \\
1861 \\
290 \\
18046 \\
3578 \\
24623 \\
13653 \\
22778\end{array}$ & $\begin{array}{r}1950 \\
5069 \\
615 \\
3661 \\
3883 \\
3532 \\
1019 \\
1054 \\
10309 \\
2232 \\
10550 \\
7059 \\
12736 \\
2268 \\
5908 \\
14216 \\
354 \\
9654 \\
12756 \\
19387 \\
1720 \\
6494 \\
7782 \\
10544 \\
8136 \\
3656 \\
22885 \\
20151 \\
3950 \\
8170 \\
28764 \\
6781 \\
20901 \\
15884\end{array}$ \\
\hline
\end{tabular}

Table 12: Table of $m, w, c, c^{\prime}, c^{\prime \prime}$ for $\operatorname{BIBD}(q, 12,1)$ construction ( $q$ composite)

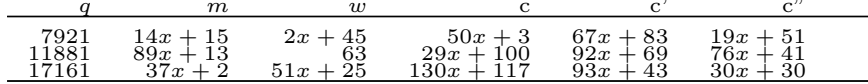


Table 13: Table of $\gamma_{1}, \ldots, \gamma_{5}$ for $\operatorname{RBIBD}(11 q+1,12,1)$ construction

\begin{tabular}{|c|c|c|c|c|c|}
\hline q & $\omega$ & $\gamma_{1}, \ldots, \gamma_{5}$ & q & $\omega$ & \\
\hline 349 & & $325,34,182,341,117$ & 373 & 2 & $343,364,164,105,245$ \\
\hline 409 & $2 \overline{1}$ & $37,118,81,266,179$ & 421 & $\overline{2}$ & $109,334,56,401,183$ \\
\hline 433 & $\stackrel{5}{*}$ & $73,243,382,155,8$ & 457 & 13 & $241,416,215,376,81$ \\
\hline $\begin{array}{l}529 \\
577\end{array}$ & 5 & $\begin{array}{r}1,239,231,284,94 \\
1,353,160,129,272\end{array}$ & $\begin{array}{l}541 \\
601\end{array}$ & $\frac{2}{7}$ & $\begin{array}{r}295,503,520,315,110 \\
433,75,128,23,478\end{array}$ \\
\hline 613 & 2 & $481,375,574,149,86$ & 625 & * & $55,494,244,399,203$ \\
\hline $\begin{array}{l}661 \\
709\end{array}$ & ${ }_{2}^{2}$ & $\begin{array}{r}25,519,178,191,344 \\
1,47,254,154,219\end{array}$ & 673 & $\begin{array}{c}5 \\
6\end{array}$ & $\begin{array}{r}403,641,129,578,358 \\
1,267,500,587,466\end{array}$ \\
\hline 757 & 2 & $283,341,615,152,424$ & 769 & 11 & $1,560,712,525,731$ \\
\hline $\begin{array}{l}829 \\
853\end{array}$ & $\stackrel{2}{2}$ & $\begin{array}{l}685,8,580,449,537 \\
97,112,176,99,779\end{array}$ & $\begin{array}{l}841 \\
877\end{array}$ & ${ }^{*}$ & $\begin{array}{l}1,423,593,86,88 \\
787,700,363,773,104\end{array}$ \\
\hline $\begin{array}{l}937 \\
997\end{array}$ & $\begin{array}{l}5 \\
7\end{array}$ & $\begin{array}{r}1 \\
223\end{array} 111,34,370,305,296$ & $\begin{array}{r}961 \\
1009\end{array}$ & 11 & $1,640,245,525,278$ \\
\hline 1021 & 10 & $9717,701,1018,506$ & 1033 & $\begin{array}{r}1 \\
5\end{array}$ & $1,166,993,401,434$ \\
\hline $\begin{array}{l}1069 \\
1117\end{array}$ & $\begin{array}{l}6 \\
2\end{array}$ & $\begin{array}{r}1,489,836,215,616 \\
05,155,382,315,578\end{array}$ & $\begin{array}{l}1093 \\
1129\end{array}$ & 11 & $\begin{array}{r}1,459,412,284,965 \\
1,1100,897,934,677\end{array}$ \\
\hline $\begin{array}{l}1153 \\
1213\end{array}$ & $\begin{array}{l}5 \\
2\end{array}$ & $\begin{array}{r}163,860,531,412,947 \\
1,220,230,105,467\end{array}$ & $\begin{array}{l}1201 \\
1237\end{array}$ & $1 \frac{1}{2}$ & $\begin{array}{l}577,977,1048,621,200 \\
1,47,110,40,1143\end{array}$ \\
\hline 1249 & 7 & $\begin{array}{r}1,358,1094,527,279 \\
65,772,831,482,1247\end{array}$ & 1297 & 10 & $1,908,1037,10,495$ \\
\hline 1381 & $\begin{array}{r}2 \\
2 \\
2\end{array}$ & $1,424,899,1202,1179$ & 1429 & 6 & $1,953,21,1180,476$ \\
\hline 1549 & 2 & $\begin{array}{l}1,1409,443,1039,279 \\
99,896,846,1391,879\end{array}$ & 1597 & 11 & $1375,976,755,1550,1395$ \\
\hline 1657 & 11 & $1156,1587,818,1451$ & 1669 & 2 & $\begin{array}{r}1549,713,1228,9,1046 \\
901,569,1292,1342,1083\end{array}$ \\
\hline 1681 & 2 & $\begin{array}{l}5,933,608,1505,652 \\
1,1353,358,932,779\end{array}$ & 1693 & 2 & $\begin{array}{r}1,206,5,724,1413 \\
1180,1124,1685,1011\end{array}$ \\
\hline 1777 & 5 & $1,730,620,1157,1047$ & 1789 & 6 & $1,1598,1036,125,81$ \\
\hline $\begin{array}{l}1801 \\
1861\end{array}$ & $1 \frac{1}{2}$ & $\begin{array}{r}1,251,273,250,1478 \\
547,4,1430,131,1125\end{array}$ & $\begin{array}{l}1849 \\
1873\end{array}$ & 10 & $\begin{array}{r}1285,1587,346,1193,584 \\
1,527,981,170,1222\end{array}$ \\
\hline 1933 & 5 & $\begin{array}{l}134,1755,743,998 \\
1,1131,800,1673,316\end{array}$ & 1993 & 5 & 37, 214, 914, 1259,591 \\
\hline 2053 & 2 & $1,562,1934,443,963$ & 2089 & $\frac{2}{7}$ & $1,1804,99,101,524$ \\
\hline $\begin{array}{l}2113 \\
2161\end{array}$ & $\begin{array}{r}5 \\
23\end{array}$ & $\begin{array}{r}1,863,15,622,1088 \\
1,273,839,2012,574\end{array}$ & $\begin{array}{l}2137 \\
2197\end{array}$ & 10 & $\begin{array}{c}9,4,1535,1732,1227 \\
1,220,305,93,980\end{array}$ \\
\hline 2209 & * & $211,94,1415,476,381$ & 2221 & 2 & $529,1709,1204,1280,1791$ \\
\hline 2293 & $\frac{2}{5}$ & $217,422,1773,2242$ & 2341 & 7 & $1129,1409,1024,591,1982$ \\
\hline 2401 & 0 & $1,2339,796,1473,1730$ & 2437 & 2 & $\begin{array}{l}2347,662,2169,1864,95 \\
1,1114,2403,1535,2138\end{array}$ \\
\hline $\begin{array}{l}2473 \\
2557\end{array}$ & $\begin{array}{l}5 \\
2\end{array}$ & $\begin{array}{r}1,657,238,1718,137 \\
1,2374,2381,1778,2085\end{array}$ & $\begin{array}{l}2521 \\
2593\end{array}$ & $\begin{array}{r}17 \overline{7} \\
7\end{array}$ & $\begin{array}{r}1,2470,2297,38,1965 \\
1,1160,2165,1264,963\end{array}$ \\
\hline $\begin{array}{l}2617 \\
2689\end{array}$ & $\begin{array}{r}5 \\
19\end{array}$ & $\begin{array}{r}1,686,1630,1485,749 \\
1267,322,965,1946,489\end{array}$ & $\begin{array}{l}2677 \\
2713\end{array}$ & $\frac{1}{5}$ & $\begin{array}{r}1,515,994,812,279 \\
1,338,27,569,1678\end{array}$ \\
\hline 2749 & ${ }_{*}^{6}$ & $1,2080,219,1118,1793$ & 2797 & 2 & $1,1853,561,1868,2080$ \\
\hline $\begin{array}{l}2809 \\
2857\end{array}$ & 11 & $\begin{array}{l}1,1406,2625,304,587 \\
1943,308,1989,1348\end{array}$ & 2833 & 5 & $1,2344,1379,1677,1250$ \\
\hline 2953 & 13 & $269,2447,2620,1268$ & 3001 & 14 & $145,1954,2895,467,1874$ \\
\hline $\begin{array}{l}3037 \\
3061\end{array}$ & 6 & $\begin{array}{r}2252,2247,989,2050 \\
, 1815,2936,827,430\end{array}$ & $\begin{array}{l}3049 \\
3109\end{array}$ & $\begin{array}{r}11 \\
6\end{array}$ & $\begin{array}{r}1,2049,1960,2336,377 \\
1,112,1707,2504,467\end{array}$ \\
\hline $\begin{array}{l}3121 \\
3181\end{array}$ & $\begin{array}{l}7 \\
7\end{array}$ & $2095,443,2019,1304,1582$ & $\begin{array}{l}3169 \\
3217\end{array}$ & $\begin{array}{l}7 \\
5\end{array}$ & $\begin{array}{l}3,1892,827,1312,723 \\
2698,2751,2513,2996\end{array}$ \\
\hline $\begin{array}{l}3229 \\
3301\end{array}$ & $\begin{array}{l}6 \\
6\end{array}$ & $\begin{array}{r}1,44,2691,143,2440 \\
1,866,1925,291,862\end{array}$ & 3253 & 2 & $865,1814,1955,2206,2535$ \\
\hline $\begin{array}{l}3301 \\
3433\end{array}$ & 22 & $\begin{array}{l}1,2420,197,2752,2505 \\
1,2795,99,1486,692\end{array}$ & 3373 & $\begin{array}{r}10 \\
5\end{array}$ & $1,2925,2231,568,722$ \\
\hline 3469 & 2 & $1,1493,1160,165,2278$ & 3481 & & $1,3100,3461,2889,2918$ \\
\hline 3517 & $\frac{2}{7}$ & $\begin{array}{l}7,3003,3452,46,1709 \\
1,794,640,1179,3197\end{array}$ & 3529 & 17 & $1909,2948,1270,1377,2411$ \\
\hline 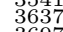 & $\frac{1}{2}$ & $1,789,2549,3556,1964$ & 3673 & 5 & $2083,3320,808,1893,3377$ \\
\hline $\begin{array}{l}3697 \\
3721\end{array}$ & 5 & $\begin{array}{l}75,536,765,448,2933 \\
124,2361,3713,2252\end{array}$ & $\begin{array}{l}3709 \\
3733\end{array}$ & $\frac{2}{2}$ & $\begin{array}{r}1,1593,1421,2644,2354 \\
1537,1287,692,2668,1829\end{array}$ \\
\hline 3769 & 7 & $1,878,2099,1624,3$ & 3793 & 5 & $57,2751,352,2597,1670$ \\
\hline $\begin{array}{l}3889 \\
388\end{array}$ & 11 & $692,2823,17469,3080$ & 4021 & 2 & $\begin{array}{l}1,1790,3167,1215,1678 \\
1,2558,3052,3009,1247\end{array}$ \\
\hline $\begin{array}{l}4057 \\
4129\end{array}$ & $\begin{array}{r}5 \\
13\end{array}$ & $\begin{array}{l}1264,387,1127,3686 \\
1828,3255,425,632\end{array}$ & $\begin{array}{l}4093 \\
4153\end{array}$ & $\frac{2}{5}$ & $\begin{array}{r}25,2,1792,2801,1047 \\
1,1299,1415,3178,500\end{array}$ \\
\hline 4177 & $\begin{array}{r}5 \\
2\end{array}$ & $545,2,323,705,2662$ & 4201 & 11 & $13,3962,3809,2830,531$ \\
\hline $\begin{array}{l}4261 \\
4297\end{array}$ & 5 & $2800,3201,2219,2270$ & 4357 & $\begin{array}{l}5 \\
2\end{array}$ & $\begin{array}{l}080,1198,513,2855 \\
1,4,3557,615,1982\end{array}$ \\
\hline 4441 & 21 & 2986 & 4489 & & 2584,1377 \\
\hline 4513 & ${ }_{17}^{7}$ & $\begin{array}{l}25,2102,1811,4372 \\
08,2697,4121,4336\end{array}$ & 4549 & 6 & $4282,2957,1934,3411$ \\
\hline 4621 & 2 & $3664,3215,999,1940$ & 4657 & 15 & $1,2140,1107,3899,788$ \\
\hline $\begin{array}{l}4729 \\
4801\end{array}$ & ${ }_{7}^{17}$ & $\begin{array}{l}916,3968,3737,1515 \\
142,2061,3377,2732\end{array}$ & $\begin{array}{l}4789 \\
4813\end{array}$ & $\frac{2}{2}$ & $\begin{array}{rl}1 & 2,3285,2801,832 \\
1,2734,2792, & 4737,971\end{array}$ \\
\hline 4861 & $1 \frac{1}{2}$ & $1,3892,1193,429,4586$ & 4909 & 6 & $1,3657,1960,1982,4511$ \\
\hline 4969 & 11 & $4172,2975,3183,3352$ & 4993 & $\frac{2}{5}$ & $1,3094,3896,2951,4479$ \\
\hline 5041 & & $139,2680,2025,2876,173$ & 5077 & $\stackrel{2}{2}$ & $4363,2,1715,4828,1323$ \\
\hline $\begin{array}{l}5101 \\
5197\end{array}$ & $\begin{array}{l}6 \\
7\end{array}$ & $\begin{array}{r}1,1317,1769,4634,100 \\
79,3987,4619,494,1990\end{array}$ & $\begin{array}{l}5113 \\
5209\end{array}$ & $\begin{array}{l}19 \\
17\end{array}$ & $\begin{array}{rl}1 & 2973,3764,232,5051 \\
837,604,4217,2217,2030\end{array}$ \\
\hline 5233 & 10 & $1,832,968,687,5063$ & 5281 & 7 & 1, $188,4061,2524,2877$ \\
\hline $\begin{array}{l}5329 \\
5437\end{array}$ & 5 & $\begin{array}{l}1184,2555,4029,4156 \\
232,3008,3165,2021\end{array}$ & $\begin{array}{l}5413 \\
5449\end{array}$ & $\begin{array}{l}5 \\
7\end{array}$ & $\begin{array}{l}1,2631 \\
1,760,4232,1893,404,2787\end{array}$ \\
\hline 5521 & 11 & $3937,3692,1384,2339,489$ & 5557 & 2 & $1,880,4976,1097,345$ \\
\hline 5641 & 14 & $5485,3910,2991,2630,2939$ & 5653 & 5 & $1,3117,1678,1199,2480$ \\
\hline $\begin{array}{l}5689 \\
5737\end{array}$ & $\begin{array}{r}11 \\
5\end{array}$ & $\begin{array}{l}1,136,1697,639,2282 \\
4415,4971,1600,5282\end{array}$ & $\begin{array}{l}5701 \\
5749\end{array}$ & $\frac{2}{2}$ & $\begin{array}{r}1,3557,188,1006,489 \\
1,5661,1186,779,1424\end{array}$ \\
\hline 5821 & $\begin{array}{l}6 \\
2\end{array}$ & $2685,4540,4892,4709$ & 5857 & 3 & $\begin{array}{l}427,4034,5650,1499,5307 \\
2425,1654,686,4481,4497\end{array}$ \\
\hline 5953 & 7 & $1743,2650,3956,1511$ & $\begin{array}{l}3001 \\
6037\end{array}$ & $\frac{1}{5}$ & $1,1485,3440,1175,868$ \\
\hline 6073 & 10 & $\begin{array}{r}3622,1227,5987,5090 \\
1,891,1270,3170,5099\end{array}$ & 6121 & 7 & $1,124,993,3716,413$ \\
\hline 6229 & 2 & $1,2,4199,1240,3321$ & 6241 & * & $3,4937,3154$ \\
\hline 6277 & $\begin{array}{r}2 \\
10\end{array}$ & $\begin{array}{r}1,2,5824,3267,4649 \\
1,836,2067,4079,280\end{array}$ & $\begin{array}{l}6301 \\
6361\end{array}$ & $\begin{array}{l}10 \\
19\end{array}$ & $\begin{array}{r}1,1556,4155,4937,3574 \\
4855,4796,5253,2434,1289\end{array}$ \\
\hline 6373 & 2 & $1,1258,2907,4874,881$ & 6397 & $\begin{array}{r}2 \\
2\end{array}$ & 1, $6074,4727,3867,1150$ \\
\hline 6481 & $\frac{6}{7}$ & $\begin{array}{l}1,5661,4244,449,2614 \\
1,4096,1629,2036,3305\end{array}$ & $\begin{array}{l}6469 \\
6529\end{array}$ & $\begin{array}{l}2 \\
7\end{array}$ & $\begin{array}{r}1,2,3831,3376,563 \\
1,5768,6208,5997,6263\end{array}$ \\
\hline $\begin{array}{l}6553 \\
6637\end{array}$ & 10 & $\begin{array}{r}1,392,2873,3322,2559 \\
3289,4095,2606,712,2129\end{array}$ & $\begin{array}{l}6577 \\
6661\end{array}$ & $\begin{array}{c}5 \\
6\end{array}$ & $\begin{array}{r}1,898,1622,5031,887 \\
1,497,4844,4798,3599\end{array}$ \\
\hline 6673 & 5 & $1,4136,3071,3357,586$ & 6709 & 2 & $847,2,4665,5237,5728$ \\
\hline $\begin{array}{l}6733 \\
6793\end{array}$ & $\begin{array}{r}2 \\
10\end{array}$ & $\begin{array}{l}1,3572,1216,795,683 \\
1,892,3839,4125,950\end{array}$ & $\begin{array}{l}6781 \\
6829\end{array}$ & $\stackrel{2}{2}$ & $\begin{array}{r}1,1016,5811,352,4763 \\
1,5482,5324,3597,5585\end{array}$ \\
\hline 6841 & 22 & $, 3201,3803,5642$ & 6889 & $*$ & $1,3446,5283,4175,4906$ \\
\hline 6949 & $\frac{2}{5}$ & $\begin{array}{l}128,6017,5026,1473 \\
928,3905,1863,5594\end{array}$ & 6961 & 13 & $1,6320,2717,6129,3862$ \\
\hline 6997 & 2 & $\begin{array}{r}1,6928,3905,1863,5594 \\
6493,845,4448,2997,1372\end{array}$ & $\begin{array}{l}7057 \\
7129\end{array}$ & 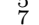 & $\begin{array}{r}1,1942,4613,1040,4353 \\
1543,4108,2876,473,5421\end{array}$ \\
\hline 7177 & 10 & $1,1964,3736,6153,5831$ & 7213 & $\frac{1}{5}$ & $\begin{array}{l}1,4108,2818,459 \\
1,3861,1618\end{array}$ \\
\hline
\end{tabular}


Table 13: Table of $\gamma_{1}, \ldots, \gamma_{5}$ for $\operatorname{RBIBD}(11 q+1,12,1)$ construction (cont.)

\begin{tabular}{|c|c|c|c|c|c|}
\hline & $\omega$ & $\gamma_{1}, \ldots, \gamma_{5}$ & q & $\omega$ & \\
\hline 7237 & 2 & $1,2,2134,2537,1911$ & 7297 & 5 & $1375,3458,1930,6143,5889$ \\
\hline 7309 & $\overline{6}$ & $4907,898,4700,3753$ & 7321 & 7 & $5764,4197,3548,5825$ \\
\hline $\begin{array}{l}7333 \\
7393\end{array}$ & 6 & $\begin{array}{l}1,4137,941,4552,5504 \\
4498,4943,4778,5529\end{array}$ & 7369 & 7 & $7051,1263,1526,2692,4943$ \\
\hline 7477 & 2 & $1,6498,4943,478,5025,2450$ & 7489 & 7 & $\begin{array}{r}5653,1490,987,7223,5278 \\
\quad 1,1520,5038,3365,4587\end{array}$ \\
\hline $\begin{array}{l}7537 \\
7561\end{array}$ & $\begin{array}{r}7 \\
13\end{array}$ & $\begin{array}{r}1,6267,6215,3376,1574 \\
1,458,1619,3015,6616\end{array}$ & $\begin{array}{l}7549 \\
7573\end{array}$ & 2 & $1,1786,2703,2840,1721$ \\
\hline 7621 & 2 & $1,3088,254,3323,945$ & 7669 & 2 & $7645,2,6417,7000,4619$ \\
\hline $\begin{array}{l}7681 \\
7741\end{array}$ & $\begin{array}{r}17 \\
7\end{array}$ & $\begin{array}{r}3517,3934,569,1628,5679 \\
1,4955,7148,514,7311\end{array}$ & $\begin{array}{l}7717 \\
7753\end{array}$ & $\begin{array}{r}2 \\
10\end{array}$ & $\begin{array}{r}1237,2283,941,3946,7352 \\
1921,6206,5854,3555, \\
1421\end{array}$ \\
\hline 7789 & 2 & $1,6980,7233,2237,4318$ & 7873 & 5 & $1,4400,4924,7773,3863$ \\
\hline 7993 & 5 & $\begin{array}{l}1,4049,5536,3638,6423 \\
1,6844,6851,1808,2697\end{array}$ & 8017 & $\frac{2}{5}$ & $\begin{array}{r}1,7618,737,2504,135 \\
1,7496,1961,5866,3873\end{array}$ \\
\hline $\begin{array}{l}8053 \\
8101\end{array}$ & $\begin{array}{l}2 \\
6\end{array}$ & $\begin{array}{r}549,2771,3478,2727,6224 \\
1,4131,484,1592,2225\end{array}$ & $\begin{array}{l}8089 \\
8161\end{array}$ & $\begin{array}{r}17 \\
7\end{array}$ & $\begin{array}{r}353,1672,795,6782,7181 \\
1,6454,2735,338,1653\end{array}$ \\
\hline $\begin{array}{l}8101 \\
8209 \\
8233\end{array}$ & 7 & $\begin{array}{l}\text { '4732, } 2573,1334,2811 \\
6124,1631,4802,6207\end{array}$ & 8221 & $\frac{1}{2}$ & $5995,2,6443,6358,3243$ \\
\hline $\begin{array}{l}8233 \\
8293 \\
8329\end{array}$ & $\begin{array}{r}10 \\
2 \\
7\end{array}$ & $\begin{array}{l}124,1631,4802,6207 \\
3910,2162,7491,2729 \\
4246,6188,5243,7797\end{array}$ & $\begin{array}{l}8269 \\
8317 \\
8353\end{array}$ & $\begin{array}{l}2 \\
6 \\
5\end{array}$ & $1,7911,8050,4281,4229$ \\
\hline 8377 & 5 & $, 2813,7341,3032,994$ & $\begin{array}{l}8353 \\
8389\end{array}$ & 6 & $1,5235,989,5518,6200$ \\
\hline 8581 & $\begin{array}{r}6 \\
6\end{array}$ & $\begin{array}{l}1,1490,40,2451,467 \\
7358,3861,3814,3305\end{array}$ & $\begin{array}{l}8521 \\
8629\end{array}$ & $\begin{array}{r}13 \\
6\end{array}$ & $\begin{array}{l}5282,1853,4060,2283 \\
3483,8468,1967,1372\end{array}$ \\
\hline $\begin{array}{l}8641 \\
8689\end{array}$ & $\begin{array}{l}77 \\
13\end{array}$ & $\begin{array}{l}6502,4083,8450,7907 \\
8086,647,2078,3951\end{array}$ & 8713 & $\frac{2}{5}$ & $\begin{array}{l}565,2,730,4833,5801 \\
7594,5624,5681,1323\end{array}$ \\
\hline $\begin{array}{l}8737 \\
8821\end{array}$ & $\begin{array}{l}5 \\
2\end{array}$ & $\begin{array}{r}7118,6209,8649,5122 \\
1,2,268,8439,3557\end{array}$ & $\begin{array}{l}8761 \\
8893\end{array}$ & $\begin{array}{r}23 \\
5\end{array}$ & $\begin{array}{r}541,3484,200,5069,5607 \\
1,2793,4130,7822,75005\end{array}$ \\
\hline $\begin{array}{l}8929 \\
9001\end{array}$ & $\begin{aligned} 11 \\
7\end{aligned}$ & $\begin{array}{r}4030,7361,5373,5336 \\
, 5152,6519,7058,449\end{array}$ & $\begin{array}{l}8941 \\
9013\end{array}$ & $\begin{array}{l}6 \\
5\end{array}$ & $1,8007,5180,3484,7109$ \\
\hline $\begin{array}{l}9049 \\
9133\end{array}$ & 7 & $4762,5943,3653,8624$ & 9109 & 10 & $1,6604,8396,9039,1835$ \\
\hline 181 & 2 & $452,6201,4996,3341$ & 9241 & 13 & $1724,9197,5931,6820$ \\
\hline $\begin{array}{l}9277 \\
9349\end{array}$ & $\begin{array}{l}5 \\
2\end{array}$ & $\begin{array}{l}4041,7637,748,1184 \\
6562,1065,446,7751\end{array}$ & $\begin{array}{l}9337 \\
9397\end{array}$ & $\begin{array}{l}5 \\
2\end{array}$ & $\begin{array}{l}1,3788,3556,4641,491 \\
1,2822,550,7071,4415\end{array}$ \\
\hline 9409 & * * & $\begin{array}{l}332,3358,6725,1899 \\
9386,658,5933,1203\end{array}$ & 9421 & 2 & $621,2,9081,4132,797$ \\
\hline $\begin{array}{l}9433 \\
9613\end{array}$ & 5 & $\begin{array}{l}386,658,5933,1203 \\
89,2,5271,310,857\end{array}$ & $\begin{array}{l}9601 \\
9649\end{array}$ & 13 & $\begin{array}{l}4052,9412,7775,759 \\
5240,4587,881,6472\end{array}$ \\
\hline $\begin{array}{l}9661 \\
9721\end{array}$ & $\stackrel{2}{7}$ & $\begin{array}{l}5992,5252,6617,6465 \\
7846,1421,7413,2000\end{array}$ & $\begin{array}{l}9697 \\
9733\end{array}$ & $\begin{array}{r}10 \\
2\end{array}$ & $\begin{array}{l}1,3818,3059,339,208 \\
1,4322,1641,8459,6400\end{array}$ \\
\hline $\begin{array}{l}9769 \\
9817\end{array}$ & $\begin{array}{r}13 \\
5\end{array}$ & $\begin{array}{l}1600,5612,1019,7893 \\
4443,5830,5894,8681\end{array}$ & $\begin{array}{l}9781 \\
9829\end{array}$ & $\begin{array}{r}\overline{6} \\
10\end{array}$ & $\begin{array}{l}1,8475,7508,2350,4859 \\
7,6986,4702,3845,1497\end{array}$ \\
\hline $\begin{array}{l}9901 \\
9973\end{array}$ & 12 & $\begin{array}{r}9133,2,7073,3873,8800 \\
5611,3093,9080,598,8957\end{array}$ & $\begin{array}{r}9949 \\
10009\end{array}$ & 11 & $\begin{array}{l}4159,2,8321,993,448 \\
7316,1401,2722,3941\end{array}$ \\
\hline 10069 & ${ }_{2}^{11}$ & $\begin{array}{l}3093,9080,598,8957 \\
1,2,9969,2854,4457\end{array}$ & $\begin{array}{l}10009 \\
10093\end{array}$ & 11 & $\begin{array}{l}7316,1401,2722,3941 \\
6584,2373,6526,8309\end{array}$ \\
\hline 10141 & 2 & $\begin{array}{l}030,6777,5642,1019 \\
5201,6704,6357,682\end{array}$ & $\begin{array}{l}10177 \\
10273\end{array}$ & $\begin{array}{r}7 \\
10\end{array}$ & $7840,7364,6545,8559$ \\
\hline 10321 & 7 & $54,1462,9093,4103$ & 10333 & 5 & $3069,3184,7643,3662$ \\
\hline $\begin{array}{l}10357 \\
10429\end{array}$ & $\begin{array}{l}2 \\
7\end{array}$ & $\begin{array}{r}6805,6860,9521,6298,10335 \\
8239,71118,3449,586,9381\end{array}$ & $\begin{array}{l}10369 \\
10453\end{array}$ & $\begin{array}{r}13 \\
5\end{array}$ & $\begin{array}{r}1 \\
325,9288,3632,3383,768 \overline{3} \\
\end{array}$ \\
\hline $\begin{array}{l}10477 \\
10513\end{array}$ & $\begin{array}{l}2 \\
7\end{array}$ & $\begin{array}{r}2185,2,3875,6699,6436 \\
8317,10300,5978,5567,8601\end{array}$ & $\begin{array}{l}10501 \\
10597\end{array}$ & $\frac{2}{5}$ & $\begin{array}{r}1,7214,10145,3610,5847 \\
1,8271,7829,572,2584\end{array}$ \\
\hline 10609 & $*$ & $1,9152,7312,1961,3687$ & 10657 & 7 & $9501,3500,8213,9712$ \\
\hline 10789 & $\begin{array}{l}7 \\
2 \\
2\end{array}$ & $\begin{array}{l}004,5758,3059,10677 \\
9370,1442,9209,9879\end{array}$ & $\begin{array}{l}10753 \\
10837\end{array}$ & $\begin{array}{r}11 \\
2\end{array}$ & $\begin{array}{l}5499,1528,7184,2345 \\
70,10661,10358,7971\end{array}$ \\
\hline $\begin{array}{l}10861 \\
10957\end{array}$ & $\frac{2}{5}$ & $\begin{array}{r}1,2,8195,562,1167 \\
1755,10048,9701,386\end{array}$ & $\begin{array}{l}10909 \\
10993\end{array}$ & $\frac{2}{7}$ & $\begin{array}{l}6082,8987,1832,3765 \\
420,10227,5674,5447\end{array}$ \\
\hline 11113 & $\begin{array}{r}13 \\
7\end{array}$ & $\begin{array}{l}93,6844,1613,3098 \\
550,9392,791,3735\end{array}$ & 11149 & 10 & $4029,7682,10691,58$ \\
\hline $\begin{array}{l}1119 \\
11317\end{array}$ & $\stackrel{2}{2}$ & $\begin{array}{l}6250,2300,7337 \\
268,11001,9497,6035 \\
\end{array}$ & 11257 & 10 & $\begin{array}{l}9 \\
3272,2537,9382,7803 \\
988,3428,7509,10997\end{array}$ \\
\hline $\begin{array}{l}11317 \\
11353\end{array}$ & 7 & $768,11001,9497,6082$ & 11437 & 2 & $7472,2321,6172,6525$ \\
\hline 11449 & 5 & $\begin{array}{l}27,6152,8884, \\
60,9599,9148,\end{array}$ & 11497 & 7 & $\begin{array}{r}6043,830,3639,7355,6376 \\
1,6992,11176,1683,7931\end{array}$ \\
\hline 11677 & 2 & $11125,8846,11608,2853,725$ & 11689 & 7 & $8396,5896,2099,1917$ \\
\hline $\begin{array}{l}11701 \\
11833\end{array}$ & $\begin{array}{l}6 \\
5\end{array}$ & $\begin{array}{l}1,2165,7785,6208,11336 \\
1,1366,3171,11348,9551\end{array}$ & $\begin{array}{l}11821 \\
11881\end{array}$ & 2 & $\begin{array}{r}2023,2487,10178,9839,7072 \\
\quad 1,8360,1149,11051,11644\end{array}$ \\
\hline $\begin{array}{l}11941 \\
12037\end{array}$ & $\begin{array}{r}10 \\
5\end{array}$ & $\begin{array}{r}5575,11150,11782,1389,8141 \\
3043,5084,3179,2932,6987\end{array}$ & $\begin{array}{l}11953 \\
12049\end{array}$ & $\begin{array}{r}5 \\
13\end{array}$ & $1494,2253,9728,8831$ \\
\hline 12073 & 7 & $\begin{array}{r}1,1480,41,7857,4634 \\
1,10953,4043,7312,950\end{array}$ & 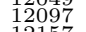 & $\begin{array}{l}10 \\
5 \\
2\end{array}$ & $1,50,10761,94,8597$ \\
\hline $\begin{array}{l}12109 \\
12241\end{array}$ & $\begin{array}{l}6 \\
7 \\
7\end{array}$ & $\begin{array}{l}953,4043,7312,950 \\
994,832,1737,8717\end{array}$ & $\begin{array}{l}12157 \\
12253\end{array}$ & ${ }_{2}^{2}$ & $\begin{array}{l}1924,9970,8391,7163 \\
14,10281,10805,10232\end{array}$ \\
\hline $\begin{array}{l}12277 \\
12301\end{array}$ & 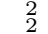 & $\begin{array}{l}7462,2415,5987,4106 \\
2,11734,2607,11957\end{array}$ & $\begin{array}{l}12289 \\
12373\end{array}$ & 11 & $\begin{array}{l}109,4172,11391,8206,11471 \\
1,962,7427,9939,11662\end{array}$ \\
\hline 12409 & 7 & $76,2885,11090,10275$ & 12421 & 7 & $4093,9155,6016,9584,4251$ \\
\hline $\begin{array}{l}12433 \\
12517\end{array}$ & $\begin{array}{r}13 \\
6\end{array}$ & 1, 10113, $8716,10997,3518$ & $\begin{array}{l}12457 \\
12541\end{array}$ & $\begin{array}{l}10 \\
14\end{array}$ & $\begin{array}{r}4225,3400,1905,6356,6401 \\
4735,3332,10397,6256,9819\end{array}$ \\
\hline $\begin{array}{l}12553 \\
12589\end{array}$ & $\begin{array}{l}5 \\
2\end{array}$ & $\begin{array}{r}1,7852,10019,8361,7826 \\
1,38,657,10762,6965\end{array}$ & $\begin{array}{l}12577 \\
12601\end{array}$ & $\begin{array}{l}10 \\
11\end{array}$ & $8671,1,4880,4864,5361,8297$ \\
\hline $\begin{array}{l}12613 \\
12697\end{array}$ & $\overline{2}$ & $422,12093,7598,10037$ & $\begin{array}{l}12637 \\
12721\end{array}$ & $\begin{array}{r}2 \\
13\end{array}$ & $1,11134,6218,5085,12263$ \\
\hline 12757 & 2 & $7,2,7505,10438,4857$ & $\begin{array}{l}12769 \\
12829\end{array}$ & 2 & $1,6497,8522,9357,6184$ \\
\hline 12841 & 21 & $590,7655,7551,8812$ & $\begin{array}{l}12829 \\
12853\end{array}$ & $\frac{2}{5}$ & $1,11871,5057,12442,1922$ \\
\hline $\begin{array}{l}12889 \\
13009\end{array}$ & $\begin{array}{r}13 \\
7\end{array}$ & $\begin{array}{l}9982,4725,2915,8180 \\
2582,3491,8548,4269\end{array}$ & $\begin{array}{l}12973 \\
13033\end{array}$ & $\begin{array}{r}14 \\
5\end{array}$ & $\begin{array}{r}1,237,12238,10664,2567 \\
11863,5006,6892,5189,5697\end{array}$ \\
\hline $\begin{array}{l}13093 \\
13249\end{array}$ & $\begin{array}{l}6 \\
7\end{array}$ & & $\begin{array}{l}13177 \\
13297\end{array}$ & $\begin{array}{l}5 \\
5\end{array}$ & $\begin{array}{l}1,11770,5831,7682,10761 \\
5,11794,6509,12243,12086\end{array}$ \\
\hline $\begin{array}{l}13249 \\
13309\end{array}$ & 6 & $639,6293,7172,9580$ & 13381 & 10 & $5,2330,586,3935,7941$ \\
\hline $\begin{array}{l}13417 \\
13477\end{array}$ & $\begin{array}{l}5 \\
2 \\
7\end{array}$ & $\begin{array}{r}1,10640,681,11686,10811 \\
9085,2,12820,8343,9995\end{array}$ & $\begin{array}{l}13441 \\
13513\end{array}$ & $\begin{array}{r}11 \\
5\end{array}$ & $\begin{array}{r}1,4600,8375,1125,4022 \\
1,7415,10028,10354,9627\end{array}$ \\
\hline $\begin{array}{l}13537 \\
13633\end{array}$ & $\begin{array}{l}7 \\
5\end{array}$ & $\begin{array}{l}542,5855,10665,7444 \\
2702,5999,238,1515\end{array}$ & $\begin{array}{l}13597 \\
13669\end{array}$ & $\begin{array}{l}5 \\
6\end{array}$ & $\begin{array}{l}1,723,4037,5978,6196 \\
1,113,2650,4083,7742\end{array}$ \\
\hline 13681 & 22 & $352,7767,11159,3458$ & 13693 & 6 & $1,7041,518,4930,4037$ \\
\hline $\begin{array}{l}13729 \\
13873\end{array}$ & $\begin{array}{r}23 \\
5\end{array}$ & $240,7221,2966,12149$ & $\begin{array}{l}13789 \\
13921\end{array}$ & 7 & $845,10700,6262,513,11525$ \\
\hline $\begin{array}{l}13933 \\
14149\end{array}$ & $\begin{array}{l}2 \\
6\end{array}$ & $\begin{array}{l}800,1613,9728,8433 \\
205,6644,5062,8819\end{array}$ & $\begin{array}{l}14029 \\
14173\end{array}$ & ${ }_{2}^{6}$ & $\begin{array}{l}1,13263,11174,1727,5836 \\
11845,2,11848,7401,9215\end{array}$ \\
\hline 14197 & 11 & $355,7076,1673,13270$ & 14221 & 6 & $10622,10594,4449,4427$ \\
\hline 14281 & $\begin{array}{l}19 \\
2\end{array}$ & $1,2,1203,7469,3478$ & 89 & 2 & $9,12825,2248,6026,11999$ \\
\hline $\begin{array}{l}14401 \\
14449\end{array}$ & $\begin{array}{l}11 \\
22\end{array}$ & $\begin{array}{l}66,7383,10816,13601 \\
3956,7576,4841,7965\end{array}$ & $\begin{array}{l}14437 \\
14461\end{array}$ & 5 & $\begin{array}{r}1,1689,13379,14314,8726 \\
1,12488,2788,2613,8105\end{array}$ \\
\hline 14533 & 2 & $\begin{array}{r}4123,2,5745,12790,9881 \\
1,376,11415,7646,689\end{array}$ & $\begin{array}{l}14401 \\
14557 \\
14629\end{array}$ & 2 & $\begin{array}{r}2107,2,706,13655,14451 \\
13699\end{array}$ \\
\hline 14641 & 5 & $8781,9004,101,5048$ & $\begin{array}{l}14629 \\
14653\end{array}$ & 2 & $\begin{array}{l}3092,300,10985,2024 \\
3092,3435,8572,5765\end{array}$ \\
\hline 14713 & 5 & $13996,6809,237,3998$ & 14737 & 10 & $1,10912,1785,2483,9980$ \\
\hline 897 & $\frac{2}{2}$ & 1, 11866, 11438, 1371, 14111 & $\begin{array}{l}14821 \\
14929\end{array}$ & 2 & $\begin{array}{l}6193,2,6934,14415,9851 \\
1,11116,5921,2294,507\end{array}$ \\
\hline 15013 & $\frac{2}{5}$ & $1104,809,8612,11577$ & 15061 & 2 & $1,13306,2066,9677,8763$ \\
\hline $\begin{array}{l}15073 \\
15193\end{array}$ & $\begin{array}{r}5 \\
5\end{array}$ & $1,2342,11585,6730,12975$ & $\begin{array}{l}15121 \\
15217\end{array}$ & $\frac{11}{10}$ & $8599,12508,11201,171,5960$ \\
\hline 15241 & 11 & $4711,10768,4910,12345,5711$ & 15277 & 6 & $13699,1173,9416,11015,7966$ \\
\hline 15349 & $\begin{array}{r}1 \\
2\end{array}$ & $1,2,2357,12945,7654$ & 15361 & & $1,9872,9783,1775,3286$ \\
\hline
\end{tabular}


Table 13: Table of $\gamma_{1}, \ldots, \gamma_{5}$ for $\operatorname{RBIBD}(11 q+1,12,1)$ construction (cont.)

\begin{tabular}{|c|c|c|c|c|c|}
\hline & $\omega$ & $\gamma_{1}, \ldots, \gamma_{5}$ & \multicolumn{2}{|l|}{ g } & $\gamma_{1}, \ldots, \gamma_{5}$ \\
\hline 15373 & 6 & $, 3592,572,11411,9159$ & 15493 & 2 & $1,3033,3436,161,10268$ \\
\hline 15541 & 6 & $1,11810,9189,1666,7157$ & 15601 & 23 & $, 5374,12056,15243,1991$ \\
\hline $\begin{array}{l}15625 \\
15661\end{array}$ & & $\begin{array}{l}1,11714,2919,5783,4030 \\
1,6602,8909,9430,669\end{array}$ & $\begin{array}{l}15649 \\
15733\end{array}$ & $1 \frac{1}{6}$ & $1,2720,13510,12225,13433$ \\
\hline 15817 & 25 & $\begin{array}{l}1,6002,8909,9430,609 \\
1,1052,7240,5391,581\end{array}$ & 15877 & $\begin{array}{r}0 \\
5 \\
15\end{array}$ & $1,10094,5013,10931,12538$ \\
\hline $\begin{array}{l}15889 \\
15913\end{array}$ & $\begin{array}{r}21 \\
5\end{array}$ & $\begin{array}{r}1735,2925,7442,11459,8176 \\
10399,10305,4072,2429,2510\end{array}$ & $\begin{array}{l}15901 \\
15937\end{array}$ & $\begin{array}{r}10 \\
7\end{array}$ & $\begin{array}{r}1,9111,2296,6194,8987 \\
1,10641,575,11120,2446\end{array}$ \\
\hline $\begin{array}{l}15973 \\
16057\end{array}$ & $\begin{array}{l}7 \\
7\end{array}$ & $\begin{array}{r}5845,5979,2168,5693,3718 \\
1,663,15773,12448,8450\end{array}$ & $\begin{array}{l}16033 \\
16069\end{array}$ & $\begin{array}{l}5 \\
2\end{array}$ & $\begin{array}{l}4669,11842,6854,13407,10481 \\
1,12688,7739,6704,3597\end{array}$ \\
\hline 16129 & $*$ & $\begin{array}{r}1,9344,7385,598,6441 \\
1,6140,12076,4419,6041\end{array}$ & $\begin{array}{l}16141 \\
16249\end{array}$ & 6 & $\begin{array}{r}1,8495,315,9118,902 \\
1,11372,14477,448,5493\end{array}$ \\
\hline $\begin{array}{l}10109 \\
16273 \\
16369\end{array}$ & 7 & $1,4628,2001,184,7301$ & 16333 & $\begin{array}{r}1 \\
2 \\
2\end{array}$ & $1,14950,15,7325,8666$ \\
\hline 16417 & 10 & $1,2384,15393,5303,9448$ & 16381 & $\begin{array}{l}2 \\
2 \\
7\end{array}$ & $\begin{array}{r}1,14,6731,4545,6412 \\
1,4082,8164,6879,4229\end{array}$ \\
\hline $\begin{array}{l}16477 \\
16573\end{array}$ & $\begin{array}{l}2 \\
2\end{array}$ & $\begin{array}{r}10039,2,13481,2194,10497 \\
5293,10796,7295,5428,15999\end{array}$ & $\begin{array}{l}16561 \\
16633\end{array}$ & $\begin{array}{r}7 \\
15\end{array}$ & $\begin{array}{l}1,14656,4364,5571,12119 \\
1,1640,14866,8649,281\end{array}$ \\
\hline $\begin{array}{l}16657 \\
16729\end{array}$ & $\begin{array}{r}\overline{5} \\
13\end{array}$ & $\begin{array}{r}9163,3088,6429,9098,16067 \\
1,4898,2003,13245,6634\end{array}$ & $\begin{array}{l}16693 \\
16741\end{array}$ & 2 & $7279,2,885,7145,8518$ \\
\hline 16921 & 17 & $13813,12920,15892,10071,14831$ & 16981 & 2 & $1,8661,3158,9869,4018$ \\
\hline $\begin{array}{l}16993 \\
17041\end{array}$ & ${ }_{7}^{10}$ & $1,11060,12281,13300,381$ & $\begin{array}{l}17029 \\
17053\end{array}$ & $\begin{array}{r}10 \\
2\end{array}$ & $\begin{array}{r}18782,3746,1115,3219 \\
3253,2,15754,9861,3029\end{array}$ \\
\hline 17077 & ${ }_{*}^{2}$ & $1,6038,10071,5512,7079$ & 17137 & $\begin{array}{r}5 \\
14\end{array}$ & $1,16760,15760,11249,16635$ \\
\hline $\begin{array}{l}17101 \\
17257 \\
17317\end{array}$ & 5 & $1,6658,13539,4748,5783$ & 17293 & 7 & $5863,9489,7738,14282,6011$ \\
\hline $\begin{array}{l}17317 \\
17377\end{array}$ & 2 & $\begin{array}{l}353,2,322,9443,12657 \\
8428,3407,1004,15147\end{array}$ & $\begin{array}{l}17341 \\
17389\end{array}$ & $\begin{array}{l}6 \\
2\end{array}$ & $\begin{array}{l}1,5409,14774,208,4139 \\
1,2,2854,16577,3381\end{array}$ \\
\hline $\begin{array}{l}17401 \\
17497\end{array}$ & $1 \frac{1}{5}$ & $228,17015,1138,13593$ & $\begin{array}{l}17449 \\
17509\end{array}$ & 14 & $\begin{array}{r}1,15778,3191,13250,63 \\
10294,12896,1955,16605\end{array}$ \\
\hline $\begin{array}{l}17569 \\
17713\end{array}$ & 11 & $\begin{array}{r}1,15652,4331,9722,549 \\
1,15436,2990,2715,6371\end{array}$ & 17581 & 10 & $55,7910,2661,731,4936$ \\
\hline $\begin{array}{l}1770 \\
17749\end{array}$ & $\begin{array}{l}1 \\
7\end{array}$ & $\begin{array}{l}10633,2,2669,184,5637 \\
2056,15212,10773,3515\end{array}$ & $\begin{array}{l}17761 \\
17929\end{array}$ & 19 & $\begin{array}{l}1,17426,8999,2062,1533 \\
1\end{array}$ \\
\hline & 2 & $\begin{array}{l}5576,16018,4529,1977 \\
7430,7739,8205,1990\end{array}$ & 17989 & 2 & $20,2092,14771,12927$ \\
\hline $\begin{array}{l}18013 \\
18061\end{array}$ & 6 & $\begin{array}{r}1,7430,7739,8205,1990 \\
, 15915,8926,12020,5873\end{array}$ & $\begin{array}{l}18049 \\
18097\end{array}$ & $\begin{array}{r}13 \\
5\end{array}$ & $\begin{array}{r}1,10544,8709,8590,16757 \\
12709,14114,16283,5379,1930\end{array}$ \\
\hline $\begin{array}{l}18121 \\
18169\end{array}$ & $\begin{array}{l}23 \\
11\end{array}$ & $11089,2092,12461,12758,16659$ & $\begin{array}{l}18133 \\
18181\end{array}$ & $\begin{array}{l}5 \\
2\end{array}$ & $\begin{array}{r}1,10671,6472,11612,9713 \\
1,14168,7018,15087,12995\end{array}$ \\
\hline $\begin{array}{l}18217 \\
18253\end{array}$ & $\begin{array}{l}7 \\
5\end{array}$ & $0,17294,2303,13581$ & $\begin{array}{l}18229 \\
18289\end{array}$ & 13 & $\begin{array}{r}8533,2,11848,15671,4065 \\
1,14810,10323,13906,17921\end{array}$ \\
\hline 18301 & 6 & $1,16839,11828,13163,15250$ & 18313 & 10 & $9547,15884,8722,3425,16113$ \\
\hline $\begin{array}{l}18397 \\
18457\end{array}$ & $\begin{array}{l}6 \\
5\end{array}$ & $\begin{array}{r}6601,16486,2271,17315,12674 \\
1,15548,15118,14975,459\end{array}$ & $\begin{array}{l}18433 \\
18481\end{array}$ & $\begin{array}{r}5 \\
13\end{array}$ & $\begin{array}{r}1,6296,14632,4815,5615 \\
6403,3824,1288,15867,5771\end{array}$ \\
\hline 18493 & 2 & $10771,3,5978,3592,15569$ & 18517 & 6 & $1,1599,18226,4508,6773$ \\
\hline 18 & 6 & $61,221,7544,14722,3453$ & 18661 & 10 & $1,12338,10119,14843,8206$ \\
\hline $\begin{array}{l}18757 \\
18793\end{array}$ & $\frac{2}{5}$ & $\begin{array}{l}1,1792,7325,18272,3021 \\
1,358,3669\end{array} 18707,18140$ & $\begin{array}{l}18769 \\
18913\end{array}$ & $\begin{array}{r}* \\
7\end{array}$ & $\begin{array}{r}1,9521,3028,18177,506 \\
511,9290,3166,8963,17445\end{array}$ \\
\hline & 2 & $1,380,16336,17909,10023$ & $\begin{array}{l}19009 \\
19009\end{array}$ & 23 & $1,15620,5146,14771,14523$ \\
\hline $\begin{array}{l}69 \\
41\end{array}$ & 2 & $\begin{array}{l}1,13610,16895,11932,16029 \\
1,1912,11381,16544,4419\end{array}$ & $\begin{array}{l}19081 \\
19213\end{array}$ & $\begin{array}{r}17 \\
5\end{array}$ & $\begin{array}{r}1,10737,1330,5606,14585 \\
1,10821,12340,13199,8636\end{array}$ \\
\hline 37 & $\overline{2}$ & $\begin{array}{r}1,17590,5793,6881,1502 \\
18127,2404,6563,285,12404\end{array}$ & 19249 & 7 & $\begin{array}{r}17905,35222,3,7727,3316 \\
1,15423,4886,9244,15077\end{array}$ \\
\hline 19321 & * & $1,140,13847,14272,14535$ & 19333 & 0 & $1,13490,1024,10773,4211$ \\
\hline $\begin{array}{l}19381 \\
19429\end{array}$ & 7 & $\begin{array}{r}11677,5315,17973,7216,18158 \\
5743,8079,3592,12839,9158\end{array}$ & 19417 & 5 & $18424,2012,4481,18915$ \\
\hline 19477 & 6 & $43,6861,8717,14786,6778$ & 19489 & 19 & $5437,15676,3167,19124,8601$ \\
\hline $\begin{array}{l}19501 \\
19609\end{array}$ & 13 & $\begin{array}{l}10852,10424,4043,17937 \\
3680,13583,6345,11188\end{array}$ & 19597 & $\stackrel{2}{11}$ & $\begin{array}{r}1,3586,17234,15971,1689 \\
6391,7420,8084,4211,5667\end{array}$ \\
\hline $\begin{array}{l}19717 \\
19777\end{array}$ & 12 & $\begin{array}{l}1,6140,3171,6964,15107 \\
, 8325,2734,14660,17975\end{array}$ & $\begin{array}{l}19753 \\
19801\end{array}$ & $\begin{array}{r}5 \\
13\end{array}$ & $91,178,14657$ \\
\hline 13 & 2 & $2,14626,18389,8979$ & 19861 & 11 & $8,12413,3136$ \\
\hline $\begin{array}{l}19993 \\
20089\end{array}$ & 10 & $\begin{array}{l}1,18056,1965,10355,7594 \\
9188,11043,10511,13660\end{array}$ & 20029 & $\begin{array}{l}2 \\
6\end{array}$ & $\begin{array}{c}1,18508,18369,18176,15869 \\
1,16719,13568,7127,808\end{array}$ \\
\hline 20113 & 10 & $3,9706,6731,16826,15807$ & 20149 & $2_{2}^{2}$ & $2209,17043,2932,14435,14480$ \\
\hline 20163 & $\begin{array}{r}13 \\
5\end{array}$ & 1, $13595,957,19078,12386$ & $\begin{array}{l}20173 \\
20269\end{array}$ & $2_{2}^{2}$ & $6487,2,11812,2315,18153$ \\
\hline $\begin{array}{l}20341 \\
20389\end{array}$ & $\begin{array}{l}2 \\
6\end{array}$ & $\begin{array}{r}1,11236,893,854,10269 \\
14961,18586,14891,17522\end{array}$ & 20353 & $\begin{array}{l}5 \\
2\end{array}$ & $1,15032,19793,8007,17332$ \\
\hline $\begin{array}{l}20521 \\
20593\end{array}$ & $1 \frac{1}{5}$ & $\begin{array}{r}9176,17734,12089,12063 \\
55,8690,6741,16883,3874\end{array}$ & 20533 & 2 & $835,2,837,6701,19036$ \\
\hline & 2 & $17659,5456,7127,13558,10533$ & 2004 & 2 & $143,2,20445,10613,12586$ \\
\hline $\begin{array}{l}20809 \\
20929\end{array}$ & $\begin{array}{l}7 \\
7\end{array}$ & $\begin{array}{r}1,8751,5450,4061,11416 \\
8965,12710,14200,4719,5543\end{array}$ & $\begin{array}{l}20857 \\
21001\end{array}$ & $\begin{array}{l}10 \\
11\end{array}$ & $\begin{array}{l}1,3880,3020,6755,9153 \\
1,8192,19427,3243,16906\end{array}$ \\
\hline $\begin{array}{l}21013 \\
21121\end{array}$ & $\begin{array}{r}2 \\
19\end{array}$ & $\begin{array}{r}1,19526,13433,6460,13209 \\
593,1706,15743,1017,14962\end{array}$ & $\begin{array}{l}21061 \\
21157\end{array}$ & $\begin{array}{l}7 \\
2\end{array}$ & $\begin{array}{r}12295,18045,7541,20530,6824 \\
17605,2,8231,2164,15663\end{array}$ \\
\hline $\begin{array}{l}21169 \\
21277\end{array}$ & $\begin{array}{r}13 \\
6\end{array}$ & $1,14103,3077,20444,21214$ & $\begin{array}{l}21193 \\
21313\end{array}$ & $1 \frac{1}{5}$ & $\begin{array}{r}1,15081,19984,14969,9062 \\
11299,10700,5242,18711,10283\end{array}$ \\
\hline 21397 & $\stackrel{2}{13}$ & $\begin{array}{r}985,2,18873,8566,15695 \\
1,9742,212,5277,18437\end{array}$ & 433 & 5 & $1,16850,9263,4066,19983$ \\
\hline 21517 & $\begin{array}{r}10 \\
5\end{array}$ & $1,5829,12400,15401,7460$ & $\begin{array}{l}21450 \\
21529\end{array}$ & 11 & $1,10156,1433,16112,9195$ \\
\hline $\begin{array}{l}21577 \\
21601\end{array}$ & $\begin{array}{r}5 \\
7 \\
14\end{array}$ & $\begin{array}{r}18757,12460,4467,9101,12650 \\
1,16888,6617,16544,21339\end{array}$ & $\begin{array}{l}21589 \\
21613\end{array}$ & $2_{2}^{2}$ & $\begin{array}{r}4573,2,3029,2158,20817 \\
1,15104,15370,17169,287\end{array}$ \\
\hline & $\begin{array}{l}14 \\
10\end{array}$ & 11875 , $14456,3910,21027,18521$ & $\begin{array}{l}21001 \\
21757\end{array}$ & 5 & $14581,5368,16677,21734,13385$ \\
\hline 21817 & 7 & $1,18436,104,5261,5673$ & 21961 & 17 & $1795,2116,14912,2199,20159$ \\
\hline 29 & 19 & $14041,1,4874,9029,5026,18837$ & & 5 & $1,8,3850,4011,20510,7019$ \\
\hline $\begin{array}{l}22189 \\
22273\end{array}$ & $\begin{array}{l}2 \\
5\end{array}$ & $\begin{array}{r}1,7724,16882,17591,11073 \\
1,19544,13019,1132,6969\end{array}$ & $\begin{array}{l}22201 \\
22369\end{array}$ & & $1,11249,1624,20144,11739$ \\
\hline 22381 & 10 & $\begin{array}{l}1,16025,2384,9027,17578 \\
1,2383,2236,13640,18659\end{array}$ & 22441 & 14 & $0280,8356,10463,135$ \\
\hline 549 & 2 & $1,2,10810,5,12015$ & & 2 & $11153,20848,20187,16532$ \\
\hline 17 & 2 & $22,11297,20823,16078$ & & 7 & $1,16178,12965,2451,7354$ \\
\hline 877 & ${ }_{2}^{7}$ & $17721,4240,21428,10949$ & 1 & & $897,6080,1936,7493,10293$ \\
\hline 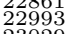 & $\frac{2}{5}$ & $8682,20870,17735,15417$ & & 5 & $12751,21578,9340,14171,20913$ \\
\hline 029 & 2 & $\begin{array}{r}1,8038,18173,4838,5859 \\
15769,2,13373,15964,19737\end{array}$ & & $\begin{array}{r}11 \\
5\end{array}$ & $\begin{array}{r}43,19196,13581,1631,9886 \\
1,14871,3874,5249,19454\end{array}$ \\
\hline 197 & 6 & $17299,2,6154,593,4785$ & 209 & 31 & $1,21868,22772,16211,18885$ \\
\hline 473 & ? & $1069,8873,3759,8386,19310$ & 23493 & 5 & $\begin{array}{l}1,4199,3183,18302,8554 \\
1,1162,18747,13277,2012\end{array}$ \\
\hline 23509 & 6 & $\begin{array}{r}1,3712,6069,7544,7571 \\
1,741,9407,17342,17794\end{array}$ & 597 & 5 & $\begin{array}{r}7423,1220,4049,2043,46 \\
14611,3658,7808,2919,15047\end{array}$ \\
\hline $\begin{array}{l}23629 \\
23689\end{array}$ & 11 & $\begin{array}{r}1,19670,11559,6641,7978 \\
4675,14126,16137,8573,1138\end{array}$ & 677 & $\begin{array}{l}5 \\
7\end{array}$ & $\begin{array}{l}1,4185,1253,23540,6112 \\
7303,10520,21059,8662,23403\end{array}$ \\
\hline 23773 & 5 & $1,21261,2458,21065,5036$ & 833 & 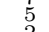 & $10393,2325,23708,575,22900$ \\
\hline 23857 & 5 & $6061,19464,16655,2025,15886$ & 869 & $\underline{2}$ & $\begin{array}{r}2707,3,20714,7547,14884 \\
1,12592,32,11897,9783\end{array}$ \\
\hline 23929 & 14 & $\begin{array}{r}1,12862,12245,21656,5739 \\
1,7892,10533,18928,7883\end{array}$ & 23977 & $\begin{array}{r}5 \\
19\end{array}$ & $\begin{array}{r}1,4822,15111,19805,21056 \\
1,5878,8816,19305,12161\end{array}$ \\
\hline 24061 & 10 & $3241,2283,9410,568,5447$ & 24097 & & $1,3206,21333,22355,4780$ \\
\hline
\end{tabular}


Table 13: Table of $\gamma_{1}, \ldots, \gamma_{5}$ for $\operatorname{RBIBD}(11 q+1,12,1)$ construction (cont.)

\begin{tabular}{|c|c|c|c|c|c|}
\hline & $\omega$ & & $g$ & $\omega$ & \\
\hline 24109 & & $9,2,13312,9659,4323$ & 24121 & 13 & $304,9305,69$ \\
\hline $\begin{array}{l}24133 \\
24181\end{array}$ & $\begin{array}{r}6 \\
17\end{array}$ & $\begin{array}{r}1,21789,10330,14027,16652 \\
22345,5319,3062,21448,5273\end{array}$ & $\begin{array}{l}24169 \\
24229\end{array}$ & $\begin{array}{r}11 \\
2\end{array}$ & $\begin{array}{l}2710,4322,181 \\
13942,22437,2\end{array}$ \\
\hline $\begin{array}{l}24337 \\
24421\end{array}$ & $\begin{array}{l}5 \\
7\end{array}$ & $\begin{array}{l}12877,21209,20643,19924,11810 \\
14677,22095,20212,11639,13538\end{array}$ & $\begin{array}{l}24373 \\
24469\end{array}$ & $\begin{array}{r}7 \\
14\end{array}$ & $\begin{array}{l}17359,21441,16814,7222,9125 \\
11395,4557,7960,21890,12587\end{array}$ \\
\hline 24481 & $1 \underset{*}{11}$ & $7729,15842,15413,4138,6429$ & 24517 & 5 & $1,22425,12299,19354,1868$ \\
\hline $\begin{array}{l}24649 \\
24709\end{array}$ & 2 & $\begin{array}{r}1,1106,12166,3785,18213 \\
1,2,21058,227,20259\end{array}$ & $\begin{array}{l}24697 \\
24733\end{array}$ & $\begin{array}{l}5 \\
2 \\
2\end{array}$ & $\begin{array}{r}1,15586,23024,6905,18753 \\
12193,2,11572,23499,22133\end{array}$ \\
\hline $\begin{array}{l}24781 \\
24841 \\
24889\end{array}$ & $\begin{array}{r}2 \\
14\end{array}$ & $\begin{array}{l}1,2,1589,7959,15688 \\
0692,4937,19959,5678\end{array}$ & $\begin{array}{l}24793 \\
24877\end{array}$ & $\begin{array}{r}5 \\
5\end{array}$ & $\begin{array}{l}15028,20913,458,12875 \\
347,23686,10361,12314\end{array}$ \\
\hline 24889 & $\begin{array}{l}11 \\
5\end{array}$ & $21154,1779,3842,3221$ & $\begin{array}{l}25033 \\
25117\end{array}$ & 5 & $\begin{array}{l}14059,12734,16510,5999, \\
2899,21746,11596,23153,10851\end{array}$ \\
\hline $\begin{array}{l}25153 \\
25237\end{array}$ & $\begin{array}{r}10 \\
2\end{array}$ & $\begin{array}{l}922,3375,21299,19274 \\
1,2,20931,8464,18839\end{array}$ & $\begin{array}{l}25189 \\
25261\end{array}$ & $\stackrel{2}{7}$ & $6979,16671,23614,16757,1676$ \\
\hline $\begin{array}{l}25309 \\
25357\end{array}$ & $\begin{array}{r}13 \\
2\end{array}$ & $\begin{array}{l}54,15556,11981,14307 \\
8664,24315,23834,227\end{array}$ & $\begin{array}{l}25321 \\
25453\end{array}$ & 19 & $1,19334,11679,15934,17501$ \\
\hline $\begin{array}{l}25537 \\
25609\end{array}$ & $\begin{array}{r}10 \\
7\end{array}$ & $\begin{array}{l}14,24802,22289,17685 \\
556,19793,21207,4214\end{array}$ & $\begin{array}{l}25561 \\
25621\end{array}$ & $\begin{array}{l}11 \\
10\end{array}$ & $\begin{array}{r}12409,9550,8277,13400,1439 \\
1,20891,7552,25305,19868\end{array}$ \\
\hline $\begin{array}{l}25633 \\
25693\end{array}$ & $\begin{array}{l}1 \\
5 \\
2\end{array}$ & $828,5614,23361,18143$ & 25657 & $\begin{array}{r}5 \\
5 \\
2\end{array}$ & $1,21400,25167,22160,269$ \\
\hline $\begin{array}{l}25741 \\
25849\end{array}$ & $\begin{array}{l}6 \\
7\end{array}$ & $\begin{array}{l}45,12700,18326,25337 \\
568,24689,9052,15837\end{array}$ & $\begin{array}{l}25801 \\
25873\end{array}$ & $\begin{array}{r}7 \\
10\end{array}$ & $\begin{array}{l}16388,22000,4937,8853 \\
026,14492,23297,24603\end{array}$ \\
\hline $\begin{array}{l}25933 \\
25981\end{array}$ & $\stackrel{2}{1}$ & $\begin{array}{l}9742,25316,6887,20073 \\
203,23690,17764,12377\end{array}$ & $\begin{array}{l}25969 \\
26017\end{array}$ & $\begin{array}{l}7 \\
5\end{array}$ & $\begin{array}{l}401,18556,1473,107,8708 \\
1,24100,21794,2673,6173\end{array}$ \\
\hline $\begin{array}{l}26029 \\
26053\end{array}$ & ${ }_{2}^{6}$ & $\begin{array}{r}1,10095,16577,194,14338 \\
1,4256,18359,23626,19989\end{array}$ & $\begin{array}{l}26041 \\
26113\end{array}$ & $\begin{array}{r}13 \\
7\end{array}$ & $\begin{array}{r}7351,6356,23291,195,21544 \\
1,404,20267,18981,6838\end{array}$ \\
\hline $\begin{array}{l}26161 \\
26293\end{array}$ & $\begin{array}{r}13 \\
6\end{array}$ & $\begin{array}{l}20210,14039,5128,615 \\
4747,2939,17884,7892\end{array}$ & $\begin{array}{l}26209 \\
26317\end{array}$ & $1 \frac{1}{6}$ & $\begin{array}{l}0,15921,24382,8369 \\
57,25868,2219,15304\end{array}$ \\
\hline $\begin{array}{l}26437 \\
26497\end{array}$ & $\begin{array}{l}5 \\
5\end{array}$ & $\begin{array}{r}1,13923,5968,8168,10313 \\
1,16004,2128,17751,19769\end{array}$ & $\begin{array}{l}26449 \\
26557\end{array}$ & $\begin{array}{l}7 \\
2\end{array}$ & $926,10301,21225,13844$ \\
\hline 26569 & & $1,9184,17289,21242,8309$ & 26641 & 7 & $, 8024,958,1053,13607$ \\
\hline $\begin{array}{l}26701 \\
26737\end{array}$ & $\begin{array}{l}22 \\
10\end{array}$ & $\begin{array}{l}855,14315,11668,19898 \\
9196,18586,1823,9927\end{array}$ & $\begin{array}{l}26713 \\
26821\end{array}$ & 10 & $607918358,4016,2579,16767$ \\
\hline $\begin{array}{l}26833 \\
26893\end{array}$ & 5 & $\begin{array}{l}5486,9711,6077,9610 \\
881,17728,5984,10295\end{array}$ & $\begin{array}{l}26821 \\
26853\end{array}$ & $1 \frac{1}{7}$ & $7219,26152,21080,3201,14627$ \\
\hline $\begin{array}{l}27061 \\
27109\end{array}$ & $\frac{2}{7}$ & $\begin{array}{r}1 \\
1,2 \\
23731,5925,5560,26255,18020,15131\end{array}$ & 27073 & 5 & $1,11402,27033,1709,10108$ \\
\hline $\begin{array}{l}27109 \\
27253\end{array}$ & $\frac{1}{2}$ & $\begin{array}{r}2 \\
5665,22814,2170,3987,9089 \\
17539,12754,1130,10427,3483\end{array}$ & 27241 & $\begin{array}{r}17 \\
6\end{array}$ & $93,25462,25349$ \\
\hline 27397 & : & $1,7960,17090,1851,23765$ & 27409 & 13 & $2234,3147,11534,10127$ \\
\hline $\begin{array}{l}27457 \\
27529\end{array}$ & 7 & $2082,2384,20241,14627$ & $\begin{array}{l}27481 \\
27541\end{array}$ & $\begin{array}{r}79 \\
19\end{array}$ & $\begin{array}{l}672,5032,26709,11057 \\
7064,16503,4121,3898\end{array}$ \\
\hline $\begin{array}{l}27673 \\
27733\end{array}$ & ${ }_{2}^{11}$ & $\begin{array}{l}9219,27218,22577,5944 \\
1,18860,4637,2458,795\end{array}$ & $\begin{array}{l}27697 \\
27793\end{array}$ & $\begin{array}{l}15 \\
5 \\
5\end{array}$ & $\begin{array}{l}9208,6747,3728,25949 \\
2662,14237,14540,4293\end{array}$ \\
\hline 27817 & 5 & $9784,9195,16067,20396$ & 27889 & $*$ & $7508,21287,6669,24338$ \\
\hline $\begin{array}{l}27901 \\
27997\end{array}$ & $\begin{array}{l}2 \\
5\end{array}$ & $\begin{array}{l}1,2 \\
1287,14716,24776,9003\end{array}$ & $\begin{array}{l}27961 \\
28057\end{array}$ & 13 & $\begin{array}{l}168,25190,10701,27629 \\
16246,6401,2619,23432\end{array}$ \\
\hline 69 & $1{ }^{7}$ & $\begin{array}{r}18055,7490,16732,21777,245 \\
1,11596,21947,10443,6530\end{array}$ & 081 & 19 & $\begin{array}{l}58,19444,18771,12197 \\
140,8865,22889,24238\end{array}$ \\
\hline 28309 & $\stackrel{2}{2}$ & 1, $20812,11993,13905,10034$ & 28393 & 15 & $509,11830,13439,21452$ \\
\hline 28413 & 2 & $700,12911,10676,26637$ & 28477 & $\frac{2}{5}$ & $27301,27338,6747,12077,22198$ \\
\hline $\begin{array}{l}28549 \\
28573\end{array}$ & ${ }_{2}^{2}$ & $\begin{array}{r}1,2,22275,20693,27376 \\
20809,3485,28118,18711,23020\end{array}$ & $\begin{array}{l}28561 \\
28597\end{array}$ & 2 & $\begin{array}{l}19040,3376,3545,4653 \\
1,2,16809,24287,27592\end{array}$ \\
\hline $\begin{array}{l}28621 \\
28669\end{array}$ & $\begin{array}{r}13 \\
6\end{array}$ & $\begin{array}{r}17455,4065,22474,5639,3656 \\
1,6525,1655,1708,7724\end{array}$ & $\begin{array}{l}28657 \\
28729\end{array}$ & $\begin{array}{r}5 \\
22\end{array}$ & $\begin{array}{l}5584,6933,26156,6371 \\
11522,40,25769,18447\end{array}$ \\
\hline $\begin{array}{l}28753 \\
28813\end{array}$ & $\begin{array}{r}10 \\
2\end{array}$ & $\begin{array}{r}1,21868,3621,19109,18422 \\
13957,7348,20667,13148,22241\end{array}$ & $\begin{array}{l}28789 \\
28837\end{array}$ & $\begin{array}{l}7 \\
2\end{array}$ & $\begin{array}{l}6149,4730,18340,21605 \\
8854,8145,12605,10928\end{array}$ \\
\hline $\begin{array}{l}28909 \\
28933\end{array}$ & $\frac{2}{2}$ & $564,23103,12556,21173$ & 28921 & 11 & $856,13097,16474,1137$ \\
\hline 29077 & ${ }_{2}^{2}$ & $\begin{array}{l}64,23103,12556,21173 \\
11452,2727,6890,4745\end{array}$ & $\begin{array}{l}29017 \\
29101\end{array}$ & $\begin{array}{l}5 \\
2\end{array}$ & $\begin{array}{l}6880,4310,18863,17817 \\
9670,5180,16611,4937\end{array}$ \\
\hline $\begin{array}{l}29137 \\
29209\end{array}$ & $\begin{array}{l}5 \\
7\end{array}$ & 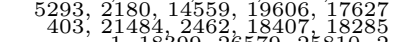 & $\begin{array}{l}29173 \\
29221\end{array}$ & 2 & $\begin{array}{rl}20887,2 & 2,4,24461,5061 \\
1,2,4534,1047,20087\end{array}$ \\
\hline $\begin{array}{l}29269 \\
29401\end{array}$ & $\begin{array}{r}6 \\
13\end{array}$ & $\begin{array}{l}1,18399,26579,25810,2 \\
9,25006,2,20847,19511\end{array}$ & $\begin{array}{l}29389 \\
29437\end{array}$ & ${ }_{2}^{2}$ & $20200,23483, \overline{2} 5$ \\
\hline 29473 & $\begin{array}{l}10 \\
10\end{array}$ & $\begin{array}{l}88,16383,11675,15206 \\
8330,6263,20703,5272\end{array}$ & 29569 & 17 & $\begin{array}{l}8040 \\
12706 \\
0329\end{array}$ \\
\hline $29+3+2$ & 10 & $\begin{array}{l}8330,6263,20703,5272 \\
18,13785,28043,26842\end{array}$ & $\begin{array}{l}29629 \\
29761\end{array}$ & 17 & $, 27987,17924,22451$ \\
\hline & $\begin{array}{l}5 \\
2\end{array}$ & $\begin{array}{l}1,14360,18965,25312,8691 \\
1,25840,2355,20915,16970\end{array}$ & $\begin{array}{l}29881 \\
29929\end{array}$ & 7 & $\begin{array}{l}27646,16509,23471 \\
13312,22868,13305\end{array}$ \\
\hline & 2 & $6793,2,26374,18707,7575$ & 30013 & 2 & $13552,8735,746,23079$ \\
\hline $\begin{array}{l}30 \\
30\end{array}$ & 10 & $\begin{array}{r}1,10612,5120,24813,2081 \\
1,15627,134,8446,28847\end{array}$ & $\begin{array}{l}30109 \\
30169\end{array}$ & 2 & $\begin{array}{l}46,11441,5241,21178 \\
52,1109,11326,11325\end{array}$ \\
\hline $\begin{array}{l}30181 \\
30253\end{array}$ & ${ }_{2}^{2}$ & $\begin{array}{r}5329,3646,7493,28820,15567 \\
1,5410,5631,19838,28133\end{array}$ & $\begin{array}{l}30241 \\
30313\end{array}$ & $1 \frac{1}{5}$ & $0472,22121,6118,5163$ \\
\hline $\begin{array}{l}30469 \\
30517\end{array}$ & ${ }_{2}^{2}$ & $\begin{array}{r}1,7562,3,317,15508 \\
24430,911,11415,4184\end{array}$ & $\begin{array}{l}30493 \\
30529\end{array}$ & $\begin{array}{r}6 \\
13\end{array}$ & $\begin{array}{r}1,27863,22742,22708,4869 \\
11581,7400,30034,6303,18545\end{array}$ \\
\hline $\begin{array}{l}30553 \\
30637\end{array}$ & ${ }_{2}^{5}$ & $\begin{array}{l}34,26147,16126,28827 \\
954,26878,6953,20757\end{array}$ & 30577 & 5 & $1,4558,19139,27513,5126$ \\
\hline 30661 & $\frac{2}{2}$ & $666,22055,9538,18693$ & 30697 & 10 & $\begin{array}{l}1572,7844,14697,17549 \\
9762,15584,26675,9249\end{array}$ \\
\hline $\begin{array}{l}30757 \\
30817\end{array}$ & $\begin{array}{l}5 \\
5\end{array}$ & 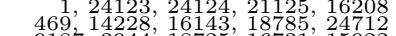 & $\begin{array}{l}30781 \\
30829\end{array}$ & 2 & $\begin{array}{r}15046,13817,27897,13208 \\
1,4270,3,14564,28931\end{array}$ \\
\hline 30841 & 7 & $9187,3944,18725,16731,15922$ & 30853 & 2 & $1,3490,13961,24177,7964$ \\
\hline 31033 & $\begin{array}{l}15 \\
10\end{array}$ & $44,20945,20439,7022$ & $\begin{array}{l}30949 \\
31069\end{array}$ & $\begin{array}{c}10 \\
2\end{array}$ & $\begin{array}{l}59,25781,17104,24860 \\
1,2, \\
10031, \\
11650,2331\end{array}$ \\
\hline 081 & $\begin{array}{r}13 \\
7\end{array}$ & $14275,1383,20852,11170,16013$ & 31189 & 10 & $\begin{array}{r}1,3080,14608,18689,15159 \\
1,20583,15280,15434,12203\end{array}$ \\
\hline 31237 & 6 & $1,15183,15184,17729,266$ & 31249 & 23 & $1,20,17470,12605,9525$ \\
\hline $\begin{array}{l}31321 \\
31357\end{array}$ & $\frac{7}{2}$ & $5869,12314,29015,9321,6490$ & $\begin{array}{l}31333 \\
31393\end{array}$ & 5 & $\begin{array}{l}1,1637,14282,30040,24449 \\
1,15572,12112,30465,29177\end{array}$ \\
\hline $\begin{array}{l}31477 \\
31513\end{array}$ & $\begin{array}{l}6 \\
7\end{array}$ & $\begin{array}{r}1,25191,4562,28817,31414 \\
1,31180,16532,21263,14661\end{array}$ & $\begin{array}{l}31489 \\
31573\end{array}$ & $\begin{array}{l}7 \\
5\end{array}$ & $\begin{array}{l}638,16187,3416,30051 \\
2119,27880,9221,23102\end{array}$ \\
\hline & 5 & $1,8804,6497,14445,31498$ & 31729 & 74 & $1548,20691,15862,5345$ \\
\hline $\begin{array}{l}31741 \\
31873\end{array}$ & 11 & $1,18861,17746,6104,6275$ & $\begin{array}{l}31849 \\
31957\end{array}$ & ${ }_{2}^{14}$ & $1135,30238,30255,4484,8837$ \\
\hline $\begin{array}{l}31981 \\
32041\end{array}$ & 6 & $\begin{array}{l}1,8270,29181,14213,19636 \\
1,23373,15395,1816,18506\end{array}$ & $\begin{array}{l}32029 \\
32077\end{array}$ & $\overline{2}$ & $13717,20672,25623,13918,11081$ \\
\hline 32089 & 13 & $44,20849,27358,3135$ & 32173 & 5 & $1,28653,12083,4312,19106$ \\
\hline $\begin{array}{l}32233 \\
32341\end{array}$ & $\begin{array}{l}5 \\
2\end{array}$ & 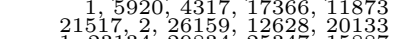 & $\begin{array}{l}32257 \\
32353\end{array}$ & $\begin{array}{l}15 \\
15\end{array}$ & $\begin{array}{r}1,15754,20342,15825,16475 \\
1,24669,8318,5614,1955\end{array}$ \\
\hline 32377 & 5 & $\begin{array}{l}1,23134,29834,25347,15887 \\
1\end{array}$ & 32401 & 7 & $1,28948,18788,25473,27107$ \\
\hline $\begin{array}{l}32413 \\
32533\end{array}$ & $\begin{array}{l}5 \\
2\end{array}$ & $\begin{array}{r}1,21225,10403,12082,19940 \\
1,25016,16137,598,7679\end{array}$ & 32497 & 7 & $92,6429,13907,7016$ \\
\hline $\begin{array}{l}32 \\
32\end{array}$ & $\begin{array}{l}2 \\
2\end{array}$ & $6118,8408,3125,13449$ & 713 & & $\begin{array}{l}943,21927,31268,32482 \\
4934,5475,19655,25154\end{array}$ \\
\hline & & & & & \\
\hline
\end{tabular}


Table 14: Table of 3960 possible exceptions in the PBD-closure of $\{7,13\}$

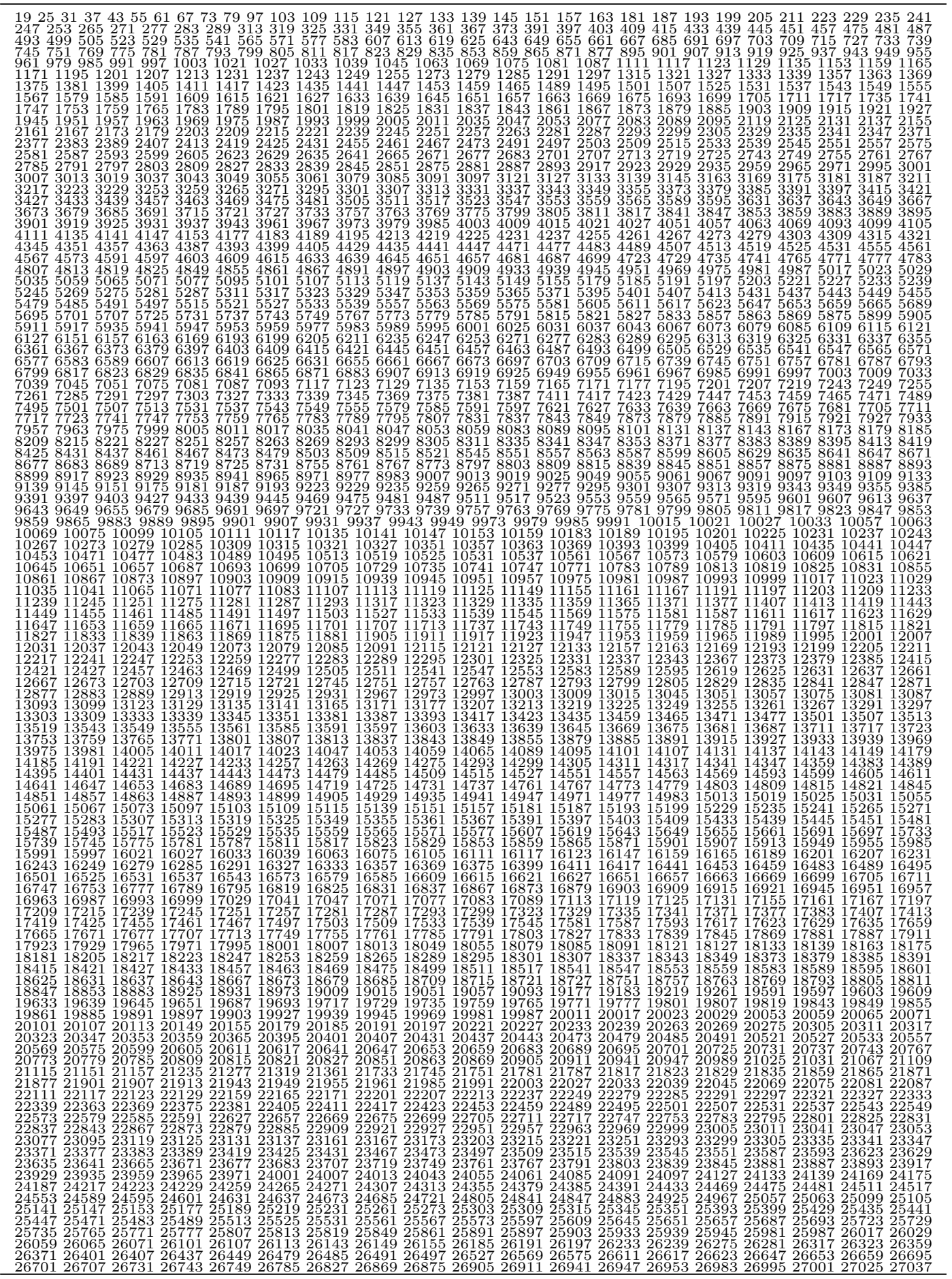


Table 14: Table of 3960 possible exceptions in the PBD-closure of $\{7,13\}$ (cont.)

\begin{tabular}{ll} 
& \\
\hline & 27043 \\
\hline
\end{tabular}

